\title{
DYNAMICS SIMULATION AND OPTIMAL CONTROL OF A MULTIPLE-INPUT AND MULTIPLE-OUTPUT BALANCING CUBE
}

\author{
A Thesis \\ presented to \\ the Faculty of California Polytechnic State University, \\ San Luis Obispo
}

\author{
In Partial Fulfillment \\ of the Requirements for the Degree \\ Master of Science in Mechanical Engineering
}

by

Felix Haimerl

June 2018 
(C) 2018

Felix Haimerl

ALL RIGHTS RESERVED 


\section{COMMITTEE MEMBERSHIP}

TITLE:

AUTHOR:

DATE SUBMITTED: June 2018

COMMITTEE CHAIR: Xi Wu, Ph.D.

Professor of Mechanical Engineering

COMMITTEE MEMBER: Eric Mehiel, Ph.D.

Professor of Aerospace Engineering

COMMITTEE MEMBER: John Ridgely, Ph.D.

Professor of Mechanical Engineering 


\begin{abstract}
Dynamics Simulation and Optimal Control of a Multiple-input and Multiple-output Balancing Cube

Felix Haimerl
\end{abstract}

This thesis document outlines the development of a multibody dynamics simulation of an actively stabilized multiple-input, multiple-output, coupled, balancing cube and the process of verifying the results by implementing the control algorithm in hardware. A non-linear simulation of the system was created in Simscape and used to develop a Linear Quadratic Gaussian control algorithm. To implement this algorithm in actual hardware, the system was first designed, manufactured, and assembled. The structure of the cube and the reaction wheels were milled from aluminum. DC brushless motors were installed into the mechanical system. In terms of electronics, a processor, orientation sensor, motor drivers, analog to digital converters, and a pulse width modulation board were assembled into the cube. Upon completion, the software to control the cube was developed using Simulink and run on a Raspberry Pi computer within the mechanism.

Keywords: LQG, LQR, Gaussian estimator, Cubli 


\section{ACKNOWLEDGMENTS}

Dr. Xi Wu, thank you for guiding me through this project and enabling me to take it on.

Dr. Eric Mehiel, thank you for teaching me all that you have about controls. Your assistance in this project was absolutely invaluable. I've learned more than I could ask for from you.

Dr. John Ridgely, thank you for being on my committee.

I would like to acknowledge the generation of PolySat mechanical engineers who taught me their ways of satellite development.

Charlie Refvem, thank you for your expertise. You were really helpful at critical moments of the project and all around a great resource to have.

Megan Rund, thanks for your help with the control algorithm development.

Max Selna and Michael Clark, thank you for your tips and tricks with CNC machining and saving me when I broke stuff. Thank you to all of the shop techs who have assisted me, you guys are great.

I would like to acknowledge the Cubli team at ETH in Switzerland for the immense inspiration.

Mama und Papa, vielen Dank für Eure Investition in meine Zukunft. 


\section{TABLE OF CONTENTS}

Page

LIST OF TABLES . . . . . . . . . . . . . . . . . . . . . . . . . . . . .

LIST OF FIGURES . . . . . . . . . . . . . . . . . . . . . . . . . . . xi

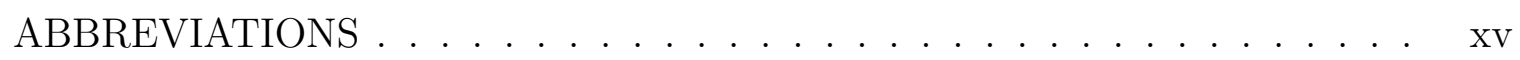
CHAPTER

1 INTRODUCTION $\ldots \ldots \ldots \ldots \ldots \ldots$

1.1 Background . . . . . . . . . . . . . . . . . . 1

1.2 Inspiration . . . . . . . . . . . . . . . . . . . 1

1.3 Objective . . . . . . . . . . . . . . . . . . . . 2

1.4 Structure of this Thesis . . . . . . . . . . . . . . . . . . . . . . . . . 2

2 SYSTEM OVERVIEW . . . . . . . . . . . . . . . . . . . . . . . . . 4

3 SYSTEM DYNAMICS SIMULATION AND ANALYSIS . . . . . . . . . . 6

3.1 Overview . . . . . . . . . . . . . . . . . . . . 6

3.2 Creating Simscape Model . . . . . . . . . . . . . . . . . . . 7

3.3 Final Simscape Model . . . . . . . . . . . . . . . . . . . . . 8

3.4 System Constraints . . . . . . . . . . . . . . . . . . . 11

3.5 Verification of System Constraints . . . . . . . . . . . . . . . . . 12

3.6 Coordinate Systems . . . . . . . . . . . . . . . . . . . . . 13

3.7 Tailoring the Model . . . . . . . . . . . . . . . . . . 14

3.7.1 Adding Sensing and Inputs . . . . . . . . . . . . . . . . . 14

3.7 .2 Modification of the Outputs . . . . . . . . . . . . 15

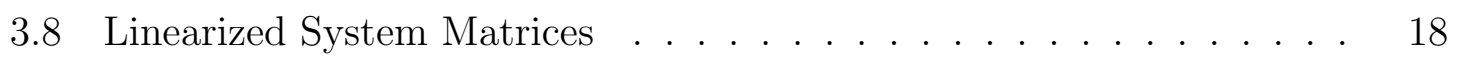

4 CONTROL ALGORITHM DESIGN . . . . . . . . . . . . . . . . . . . . . 20

4.1 State Space Vector and Controlled Output Vector . . . . . . . . . . . 20

4.2 Control Algorithm Architecture . . . . . . . . . . . . . . . . . . 22

4.3 Linear Quadratic Regulator . . . . . . . . . . . . . . . . . . . 24

4.4 State Estimator $\ldots \ldots \ldots$. . . . . . . . . . . . . . . . . . . . . . 27

4.5 Implementation of the Control Algorithm into the Simulation . . . . 30

5 MECHANICAL DESIGN . . . . . . . . . . . . . . . . . . . . . . . . . 31 
5.1 System Architecture $\ldots \ldots \ldots \ldots$. . . . . . . . . . . . . . 31

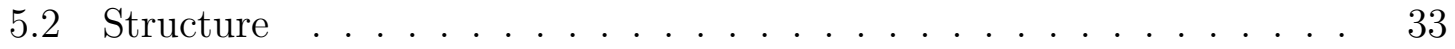

$5.2 .1 \quad$ Panel Layout . . . . . . . . . . . . . . . . . . . . . 33

5.2 .2 Joints $\ldots \ldots \ldots \ldots \ldots \ldots \ldots \ldots$

5.2 .3 Datum Structure . . . . . . . . . . . . . . . . . 38

5.2 .4 Gaps and Screw Clearance . . . . . . . . . . . . . . . 39

5.2 .5 Side Panels . . . . . . . . . . . . . . . . . 40

5.2 .6 Acrylic Panels . . . . . . . . . . . . . . . . . . . 43

5.3 Motor Mounts . . . . . . . . . . . . . . . . . . . . . . . . . . 45

5.4 Wheel Stack Design . . . . . . . . . . . . . . . . . . . . . 46

6 ELECTRICAL DESIGN . . . . . . . . . . . . . . . . . . . . . 48

6.1 Architecture . . . . . . . . . . . . . . . . . . . 48

6.2 Computer . . . . . . . . . . . . . . . . . . . . . . . . . 49

6.3 Motors . . . . . . . . . . . . . . . . . . . . . . . . . . . . . . 49

6.4 Motor Drivers $\ldots \ldots \ldots \ldots$

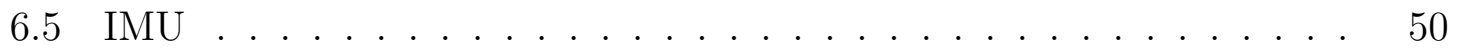

6.6 ADC Board . . . . . . . . . . . . . . . . . . . . . 51

6.7 PWM Board . . . . . . . . . . . . . . . . . . . . . . . 51

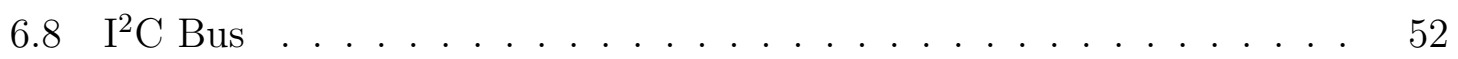

7 MANUFACTURING AND ASSEMBLY $\ldots \ldots \ldots \ldots \ldots$

7.1 Overview . . . . . . . . . . . . . . . . . . 53

7.2 CNC Machining . . . . . . . . . . . . . . . . . . . 53

7.2 .1 Toolpath Planning and G-code Generation . . . . . . . . . . 53

7.2 .2 Manufacturing Reaction Wheels . . . . . . . . . . . . . 54

$7.2 .3 \quad$ Manufacturing Side Panels and Motor Mounts . . . . . . . . 56

7.2 .4 Motor Mounts . . . . . . . . . . . . . . . . . . . . 59

$7.2 .5 \quad$ Fixturing $\ldots \ldots \ldots \ldots \ldots \ldots$

7.2 .6 Lessons and Problems . . . . . . . . . . . . . . . . 64

7.3 Wheel Stack Assembly . . . . . . . . . . . . . . . . . . . . . 69

$7.4 \quad$ Acrylic Panels . . . . . . . . . . . . . . . . . . . . . . . . . 71

7.5 Cables . . . . . . . . . . . . . . . . . . . . . 72

8 SOFTWARE AND MECHATRONIC IMPLEMENTATION . . . . . . . . 73 
8.1 Software Overview . . . . . . . . . . . . . . . . . . . 73

8.2 Use of Simulink Stateflow for Sequential Program Execution . . . . . 73

8.3 Control Software . . . . . . . . . . . . . . . . . . . . . . . 75

8.3 .1 Motor Drivers . . . . . . . . . . . . . . . . . . . 76

8.3 .2 Reading Motor Speeds . . . . . . . . . . . . . . . . 77

8.3 .3 Sensing Orientation . . . . . . . . . . . . . . . 80

8.3 .4 Sensing Rotational Rates . . . . . . . . . . . . . . . . . . . 83

8.4 Emergency Stop . . . . . . . . . . . . . . . . . . . . . . . . . 84

8.5 Testing $\ldots \ldots \ldots \ldots \ldots \ldots \ldots$

8.5 .1 Cube's Orientation . . . . . . . . . . . . . . . . 85

8.5 .2 Direction of Angular Rate . . . . . . . . . . . . . 86

8.5 .3 PWM Generation . . . . . . . . . . . . . . . . . . 86

$8.5 .4 \quad$ Wheel Spin Direction $\ldots \ldots \ldots \ldots$. . . . . . . . 87

8.5 .5 Wheel Identification . . . . . . . . . . . . . . 87

9 ADJUSTMENT OF CONTROL ALGORITHM TO OPERATE ON HARD-

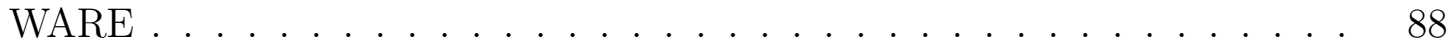

9.1 Process . . . . . . . . . . . . . . . . . . . . . . . . . 88

9.2 Results . . . . . . . . . . . . . . . . . . . . . . . . . . . . . . . . 89

10 RESULTS $\ldots \ldots \ldots \ldots$

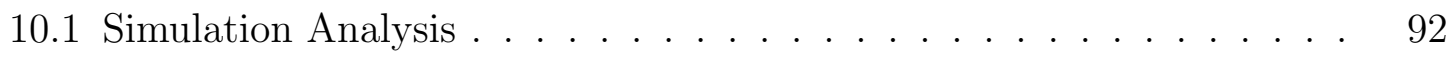

10.1.1 Reaction Wheel Mass Properties . . . . . . . . . . . . 92

10.1 .2 Cube Mass Properties . . . . . . . . . . . . . . . . 94

10.2 Control Algorithm Analysis _ . . . . . . . . . . . . . . . 95

10.2.1 Functionality of Control Algorithm in Simulation . . . . . . 95

10.2.2 Functionality of Control Algorithm in Hardware . . . . . . . 96

10.2 .3 Analysis of the Discrepancy $\ldots \ldots \ldots$

10.3 Future efforts . . . . . . . . . . . . . . . . . . . . . . . . 101

10.3.1 Adjustment of Control Logic to Balance the Hardware . . . . 101

10.3 .2 Refinement of Simulation . . . . . . . . . . . . . . . . 102

10.3 .3 Refinement of Control Algorithm . . . . . . . . . . . 103

10.3 .4 Refinement of Software . . . . . . . . . . . . . . . . . 103

10.3 .5 Refinement of Hardware . . . . . . . . . . . . . . . 103 


\section{APPENDICES}

A $\quad$ Matlab Code . . . . . . . . . . . . . . . . . . . . . . . . 109 


\section{LIST OF TABLES}

Table

Page

10.1 Comparison of mass properties of reaction wheels used in simulation and measured from hardware. . . . . . . . . . . . . . . . . . . 93

$10.2 \quad$ Mass difference between the simulation and hardware. . . . . . . . 94 


\section{LIST OF FIGURES}

Figure

2.1 The system consists of a cube shaped robot with three internal reaction wheels and electronics. . . . . . . . . . . . . . . . 4

$3.1 \quad$ 3D animation generated by Simscape. . . . . . . . . . . . . . . . 6

$3.2 \quad$ Final Simscape model of the cube. . . . . . . . . . . . . . . . . . . 8

3.3 Spherical constraint of the simulation. Its base coordinate system is coupled to that of WheelPanel-X-3 while its follower coordinate system connects to the global reference frame of the simulation. Q represents the orientation of the base CSYS relative to the follower CSYS. Wx, wy, and wz are the rotational velocities of the WheelPanel-X-3. 9

3.4 Each purple area of the simulation contains the mechanical parts included in a wheel panel assembly, including a panel, a motor mount, the reaction wheel, the mounting hub, and screws. . . . . . . . . . . 10

3.5 The red areas of the simulation contains the blocks representing the mechanical parts which mount to an electronics-bearing side panel. The large square to the left is the aluminum side panel, the one to the right is the acrylic panel, and the other blocks are screws and electronics. . . . . . . . . . . . . . . 10

$3.6 \quad$ Motion of the cube in the simulation with no inputs. . . . . . . . . 12

$3.7 \quad$ Illustration of the cube with its three coordinate systems . . . . . . 13

3.8 Portion of the model responsible for finding the cube's angles $\theta_{\text {cube }}$ and $\phi_{\text {cube }}$ from the quaternion output of the spherical constraint block. 16

$3.9 \quad$ Graphical explanation of $\theta_{\text {cube }}, \phi_{\text {cube }}, r_{g, B O D Y}$, and $r_{g, W O R L D} \cdot$. . . 17

3.10 Coordinate transformation of angular rates from $C S Y S_{S E N S O R}$ to $C S Y S_{B O D Y} \ldots \ldots \ldots \ldots \ldots$. . . . . . . . . . . . . . . . . .

3.11 The subsystem containing the Simscape model has three inputs which feed the cylindrical constraints and six outputs, three wheel velocities, two cube angles, and one cube rotational velocity. The symbols $\begin{array}{ll}\text { above the signals represent their declaration as linear analysis points. } & 19\end{array}$

4.1 Control logic of the cube. The difference between $y_{\text {ref }}$ and $y$ is fed into a state estimator where the full state $x$ is estimated. The estimate $\hat{x}$ is then multiplied by the gain matrix and the result sent to the plant as the motor torques. . . . . . . . . . . . . . 23 
$4.2 \quad$ Modeling of noise in control systems. . . . . . . . . . . . . . . .

5.1 CAD model of the cube. The yellow components are the reaction wheels, the motor mounts are dark gray, the stator and rotor of the motors are green and torquoise, the Raspberry Pi is orange, and the motor drivers are shown in black. . . . . . . . . . . . . . . . . . 31

$5.2 \quad$ Explanation of minimum cube size given the usage of EC45 motors. 32

$5.3 \quad$ Side panels are datum-less if the edges are cut to 45 degree angles. 34

$5.4 \quad$ Two side panels are datum-less if they do not extend to the edges. 35

$5.5 \quad$ Final side panel layout for the cube . . . . . . . . . . . . . . . . 36

$5.6 \quad$ Expected results of using seperate blocks for the corners. . . . . . . 37

5.7 Datum structure on a master panel . . . . . . . . . . . . . . . . 38

$5.8 \quad$ Zoomed in screenshot of the gaps between the side panels. . . . . . 39

$5.9 \quad$ Final design of the $+\mathrm{X}$ side panel. . . . . . . . . . . . . . . . . . 40

$5.10 \quad$ Final design of the $-\mathrm{x}$ side panel. . . . . . . . . . . . . . . 41

$5.11 \quad$ Final design of the + z side panel. . . . . . . . . . . . . . . . . 42

$5.12 \quad$ Final design of the $-\mathrm{z} / \mathrm{-y}$ side panels. . . . . . . . . . . . . . . . 43

5.13 CAD model of the acrylic panel which the three ADCs, the IMU, and one of the motor drivers mounts to. . . . . . . . . . . . . . . . 44

5.14 CAD model of a motor mount. . . . . . . . . . . . . 45

5.15 Wheel stack design, showing the motor, motor mount, mounting hub, reaction wheels, bearing, and side panel. . . . . . . . . . . . . . 47

6.1 The overall electrical system architecture of the balancing cube. Note that the system contains three motors and motor drivers. Only one set is depicted. . . . . . . . . . . . . . . . . 48

6.2 Pinout diagram of the ESCON $36 / 3$ motor drivers. . . . . . . . . . 50

$7.1 \quad$ Reaction wheel work piece after first machining job. . . . . . . . . . 54

$7.2 \quad$ Finished reaction wheels. . . . . . . . . . . . . . . . . 56

$7.3 \quad$ A wheel panel after the adaptive clearing operation. The lines in the aluminum show the path of the tool. . . . . . . . . . . . . 58

$7.4 \quad$ Finished wheel panel. . . . . . . . . . . . . . . . . 59

$7.5 \quad$ Picture of reaction wheel fixture showing the datums. . . . . . . . . 61 
7.6 Illustration of the modification of the panel fixture. The minor panels occupied the red area. The fixture was then cut larger to accommo$\begin{array}{ll}\text { date the major panels which occupied both the red and blue areas. } & 62\end{array}$

$7.7 \quad$ Side panel fixture with datums. . . . . . . . . . . . . . . 63

$7.8 \quad$ Motor mount fixture with datums. . . . . . . . . . . . . . . . . . . 64

$7.9 \quad$ Screenshot of HSMWork's representation of a chamfer mill. . . . . . 66

$7.10 \quad$ Location of undesired internal radii on master panel are highlighted in red. . . . . . . . . . . . . . . . . 67

7.11 Graphical explanation of the issue of internal radii in the side panel and the solution to the problem. . . . . . . . . . . . 68

\begin{tabular}{lll}
7.12 & Picture of the motor shaft following the modification done for the & \\
\hline & set screw. . . . . . . . . . . . . . . . . . . . 70
\end{tabular}

7.13 Finished wheel stack assemblies. . . . . . . . . . . . . . . . . . . . . 71

$8.1 \quad$ Stateflow diagram of the software running on the Raspberry Pi. . . $\quad 74$

$8.2 \quad$ Simulink model of the control algorithm running on the cube. The subsystem contains the programs for actuation and sensing. . . . . 75

8.3 The portion of the program which commands the motor drivers based on a torque input. . . . . . . . . . . . . . 76

8.4 The $\mathrm{I}^{2} \mathrm{C}$ write function which commands the ADC board to save its channel 0 translation results in the output register. . . . . . . . . . 78

$8.5 \quad$ The $\mathrm{I}^{2} \mathrm{C}$ read function which reads from the output register of the ADC. . . . . . . . . . . . . . . . . . 79

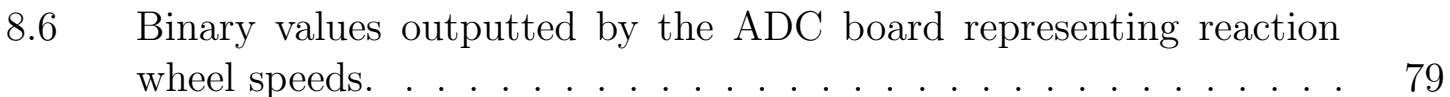

8.7 Portion of the program which senses the orientation of the cube, derives the angles $\theta_{\text {cube }}$ and $\phi_{\text {cube }}$, and post-processes the data. . . . 80

8.8 Cluster of Simulink blocks which read the orientation and find $\theta_{\text {cube }}$ and $\phi_{\text {cube }}$ based on it. . . . . . . . . . . . . . . . . . . . . . . . . 80

$8.9 \quad$ Sample signal before it was post processed. . . . . . . . . . . . . . . 81

$8.10 \quad$ Post-processing filter logic. . . . . . . . . . . . . . . . . . . . 81

8.11 Sample signal after it was post processed. . . . . . . . . . . . . . 83

8.12 Portion of the program responsible for sensing the rotational speed of the cube, converting the readings to $C S Y S_{B O D Y}$, and post-processing

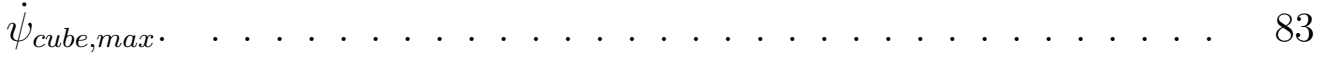


8.13 The simulated values and hardware measurements of $\theta$ and $\phi$ throughout the same motion. The similarity in the data verified that the sign convention of the angles matched. . . . . . . . . . . . . . . . 86

9.1 Cube orientations $\theta$ and $\phi$ and rate of rotation $\dot{\psi}$ as calculated by the discrete simulation with a linearized plant model. . . . . . . . . 90

9.2 Reaction wheel speeds as calculated by the discrete simulation with a linearized plant model. . . . . . . . . . . . . . . . . . . 91

10.1 Given a known input torque, the angular velocities of the reaction wheels were measured to find their inertias. . . . . . . . . . . . . . 93

10.2 The angle $\theta$ of the cube as it falls from its upright position into the same direction as calculated by the simulation and as measured from actual hardware. . . . . . . . . . . . . . . . . . . . 94

10.3 Simulated orientation of the cube, given by $\theta$ and $\phi$, and rate of rotation $\psi$. . . . . . . . . . . . . . . . . . . . . . . . . . . . 95

10.4 Simulated rotational rates of the three reaction wheels. . . . . . . . 95

10.5 Angles $\alpha$ and $\beta$ and rotational rate $\dot{\psi}$ of the cube during active control. The device was held in place to keep it from falling over. . 97

10.6 Speeds of the three reaction wheels during an active balancing attempt of the cube. . . . . . . . . . . . . . . . . 98

10.7 The error signal as calculated by the control loop during operation on the hardware. . . . . . . . . . . . . . . . . . . . . . 99

10.8 The state estimation as calculated by the control loop during operation on the hardware. . . . . . . . . . . . . . . . . . . . . . . . 99 99

10.9 Results of the multiplication of the gain L by the error vector. Note that the scales are different. . . . . . . . . . . . . . . . . . . . 100

10.10 Comparison of the error vector as calculated by the simulation and as measured by the hardware. The discrepancy between the two signals is likely the cause for the divergence of the state estimator. . 101 


\section{ABBREVIATIONS}

$\begin{array}{ll}\text { ADC } & \text { Analog to Digital Converter } \\ \text { CAM } & \text { Computer Aided Manufacturing } \\ \text { CNC } & \text { Computer Numerical Controll } \\ \text { CSYS } & \text { Coordinate System } \\ \text { DOF } & \text { Degree(s) of Freedom } \\ \text { IMU } & \text { Inertial Measurement Unit } \\ \text { I2C } & \text { Inter-integrated chip (communication protocol) } \\ \text { LQR } & \text { Linear Quadratic Regulator } \\ \text { MPU } & \text { Micoprocessor Unit } \\ \text { PWM } & \text { Pulse Width Modulation }\end{array}$




\section{Chapter 1}

\section{INTRODUCTION}

\subsection{Background}

Inverted pendulums, such as the reaction wheel based cube, are inherently unstable in their inverted positions due to their centers of gravity being above the pivot point. They require active input to balance, which is commonly provided in the form of a

closed-loop feedback controller [1]. Because of this instability, inverted pendulums are often used to test and demonstrate control algorithms focused on stabilization.

An example of a simple inverted pendulum is a rod attached to a bearing with an actuator applying torque to the rod. This type of system has one degree of freedom and one input, making it a single-input, single-output, or SISO system. A feedback control algorithm is usually developed by proper pole placement.

The reaction wheel based, cube shaped inverted pendulum has three rotational degrees of freedom and three inputs, making it a multi-input, multi-output, or MIMO system. The inputs and outputs area coupled, making control gain design through pole placement next to impossible. This type of system was considered to be appropriate for learning the mechanisms of optimal control algorithm design and implementation.

\subsection{Inspiration}

This project was inspired by the Cubli, a reaction wheel based balancing cube developed by a team of engineers and researchers at the ETH in Zurich, Switzerland [2]. The Cubli team is credited for the conceptual design of the robot and the idea to use 
a LQR control algorithm to balance the device.

The balancing cube was also heavily inspired by CubeSat satellites. Reaction wheels, after all, are most commonly used to orient spacecraft in their orbits. Exposure to these mechanism and the control algorithms involved motivated the development of the cube. The mechanical structure has ties to previous satellite structures developed by California Polytechnic State University's PolySat research laboratory.

\subsection{Objective}

The objectives of this project were to develop a realistic, non-linear multibody simulation and a control algorithm capable of balancing the inverted pendulum on a corner. To verify the functionality of the control algorithm, the plan was to implement it into a real system.

This specific mechanism was chosen due to its demand of a broad portfolio of skills. Some of these skills were brushed up and expanded upon, such as the mechanical design aspect and CNC milling, but other areas such as multibody dynamics simulations, control algorithm design, and implementation of the control logic into a mechatronic system were new skills developed during this project. The cube, therefore, was the result of the culmination of skills learned previously and a platform to develop new abilities. Learn by doing.

\subsection{Structure of this Thesis}

This document describes the process of developing the dynamics model, control algorithm, and the real system. Chapter 2, System Overview, gives a brief explanation of the mechatronic system in question including the inputs and outputs, the mechanical design, and the electrical design. This serves to give the reader context for Chapter 
3. which describes the process of creating a simulation of the dynamics of the system. Chapter 8.3 then discusses the development of the control algorithm and state estimator and their implementation into the Simulink simulation. Chapters 5 and 6 are an explanation of the design process and final design of the hardware. Chapter 7 gives an account of the manufacturing process. Chapter 8 is lays out the development of the code which runs in the cube. Chapter 9 is an account of the changes that had to be made to implement the control algorithm into the hardware. Finally, the results of the work are presented in Chapter 10. 
Chapter 2

\section{SYSTEM OVERVIEW}

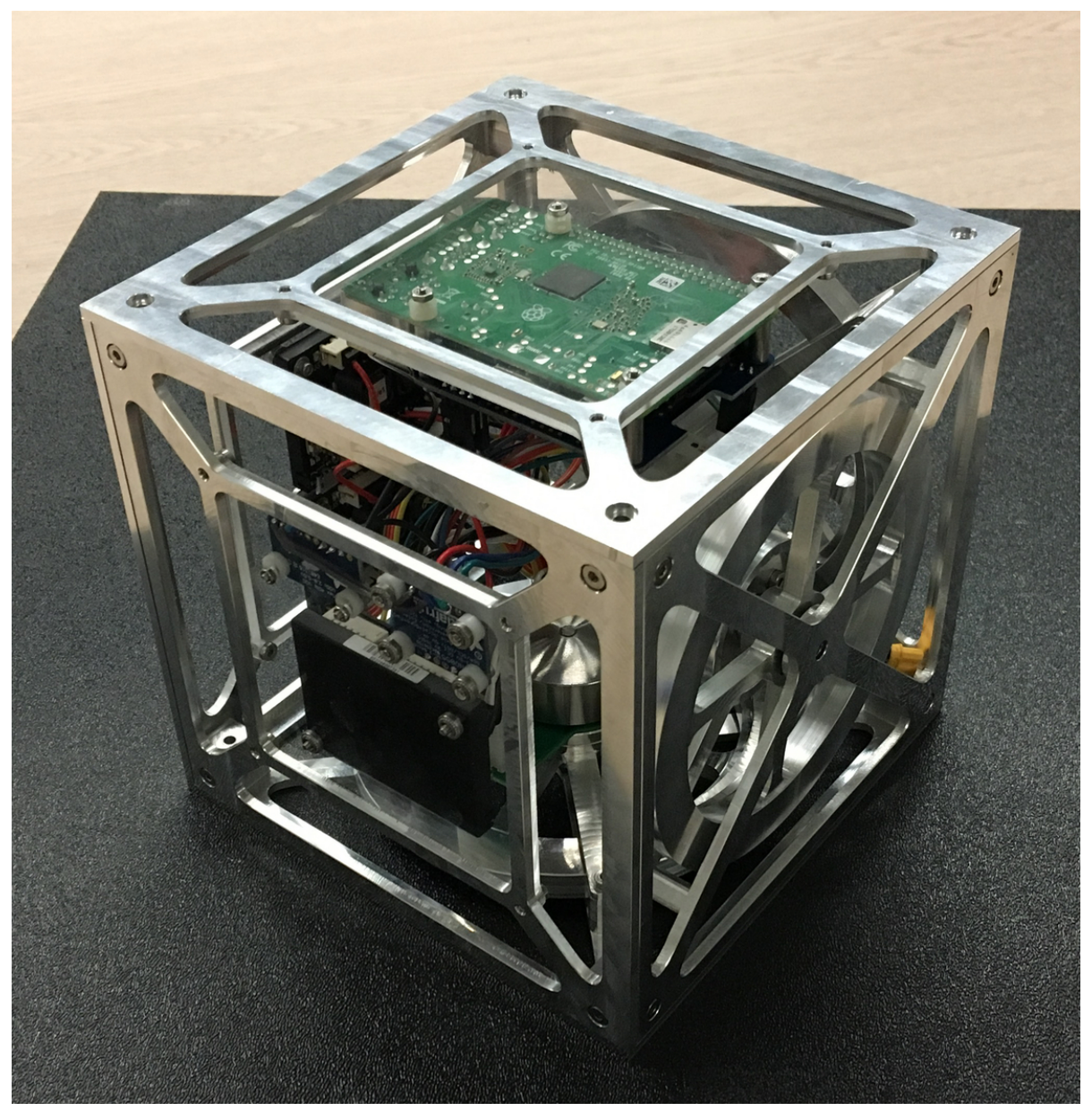

Figure 2.1: The system consists of a cube shaped robot with three internal reaction wheels and electronics.

This chapter introduces the mechatronic system for which the simulation and control algorithm were developed.

The cube has sides of $15 \mathrm{~cm}$. Its contains three reaction wheels mounted perpendicular to each other, with each one being parallel to one of the outer surfaces of the cube. The design of the structure consists of six panels, each of which makes up one 
side of the cube.

The three reaction wheels are attached to three of the panels, the vertex of which is the balancing point of the cube. This device is designed to only balance on this specific corner for several reasons. The first is that the cube's center of gravity is lowest when this is the case. This makes the system less sensitive to the effects of gravity. Second, the cube can only have its three reaction wheels at the top or at the bottom since the device would otherwise be tilted significantly. Finally, to simplify the control algorithm and software implementation it was decided to only balance the cube on one corner.

In terms of electronics, the cube has three brushless dc motors with hall sensors controlled by closed-loop current control drivers. A Raspberry Pi executes the control algorithm. An IMU provides the orientation and rotational rates of the cube, and a PWM board and three ADCs convert between electrical signal types. Chapter 6 describes the electrical design in detail.

Three angles are necessary to describe the orientation of the system - two of them measure how much the cube has tilted from its intended position, the third measures the angle of the cube about the axis which coincides with the top and bottom corner. A torque applied to the structure by each of the reaction wheels causes a change in all three angles, making this system heavily coupled. 


\section{SYSTEM DYNAMICS SIMULATION AND ANALYSIS}

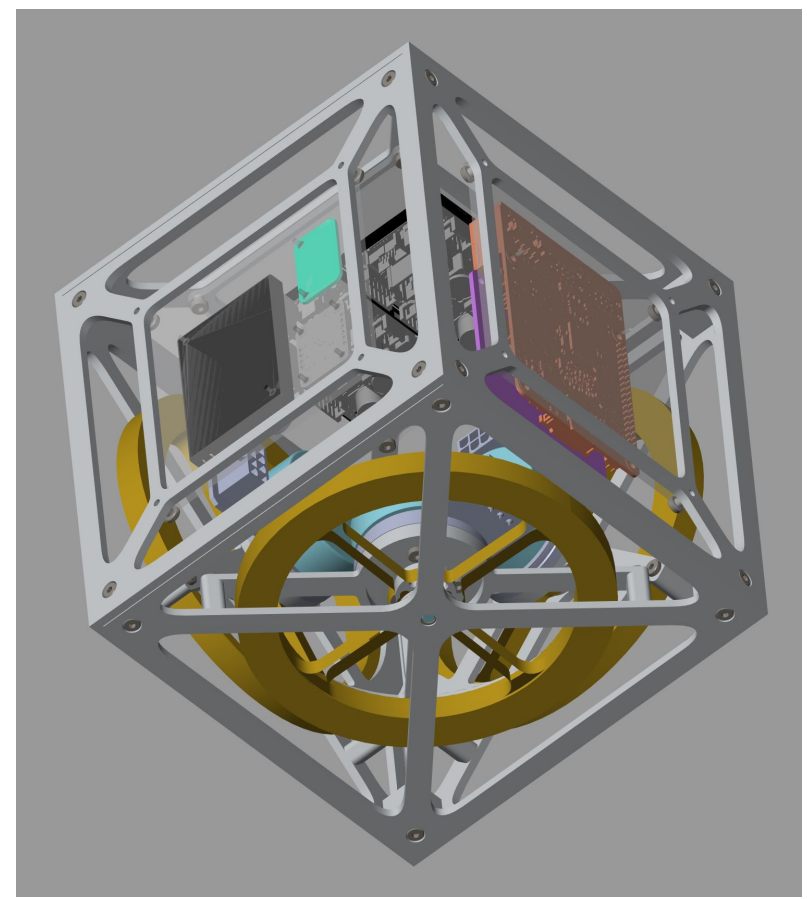

Figure 3.1: 3D animation generated by Simscape.

\subsection{Overview}

The cube's dynamics were modeled using MathWork's SimScape multibody dynamics software, which is an add-on to Simulink. The simulation was created graphically on a canvas using blocks to represent various rigid bodies, mechanical constraints, and mathematical operations and lines to represent information flow between blocks.

MathWorks offers a tool which exports complete mechanical assemblies from CAD software packages and then imports this information into Matlab. This functionality was used to create an initial simulation. Then, inputs and outputs were added to read the orientation of the cube and the reaction wheels' velocities and to control 
the input torque. The outputs were augmented with coordinate transformations and geometry calculations so that the desired orientation parameters could be measured. Finally, the system was linearized about the balancing point.

\subsection{Creating Simscape Model}

As mentioned above, MathWorks offers a tool which installs into several CAD packages [3]. In this case, the tool for SolidWorks was used. Upon installation an additional menu item appears in SolidWorks.

This export tool was used to bundle the information of the mechanical assembly into a format which Matlab understands. It created .step files which contain the geometric information associated with each of the individual parts in the assembly, a file which lists the details of all coordinate systems including the coordinate system of each part as well as the coordinate system of the main assembly. Another aspect of the assembly that this tool exported were the material properties that describe the individual parts in this assembly. Finally, the constraints (called "mates" in SolidWorks) which were defined in SolidWorks were also pushed to a file. A master file containing information of how to put all of this content back together, also appeared automatically.

The next step to creating the simulation was to enter the command smimport('FileName.xml') into Matlab. This command generates the graphical cluster of blocks and lines which represent the mechanical system. Additional efforts transformed the simulation from its auto-generated organization to the desired structure. The result is presented in Section 3.3 .

At this point the simulation was functional, meaning it could be used to analyze the motion of the dynamic system. Simscape created a 3D rendering of the mechanism. At this point, no control law had been incorporated so the system acted as a 
combination of masses constrained to each other and being influenced by gravity.

\section{ADD GRAPHS OF UNCONTROLLED SIMSCAPE MODEL HERE.}

\subsection{Final Simscape Model}

This section offers a breakdown of the Simscape model shown in Figure 3.2 .

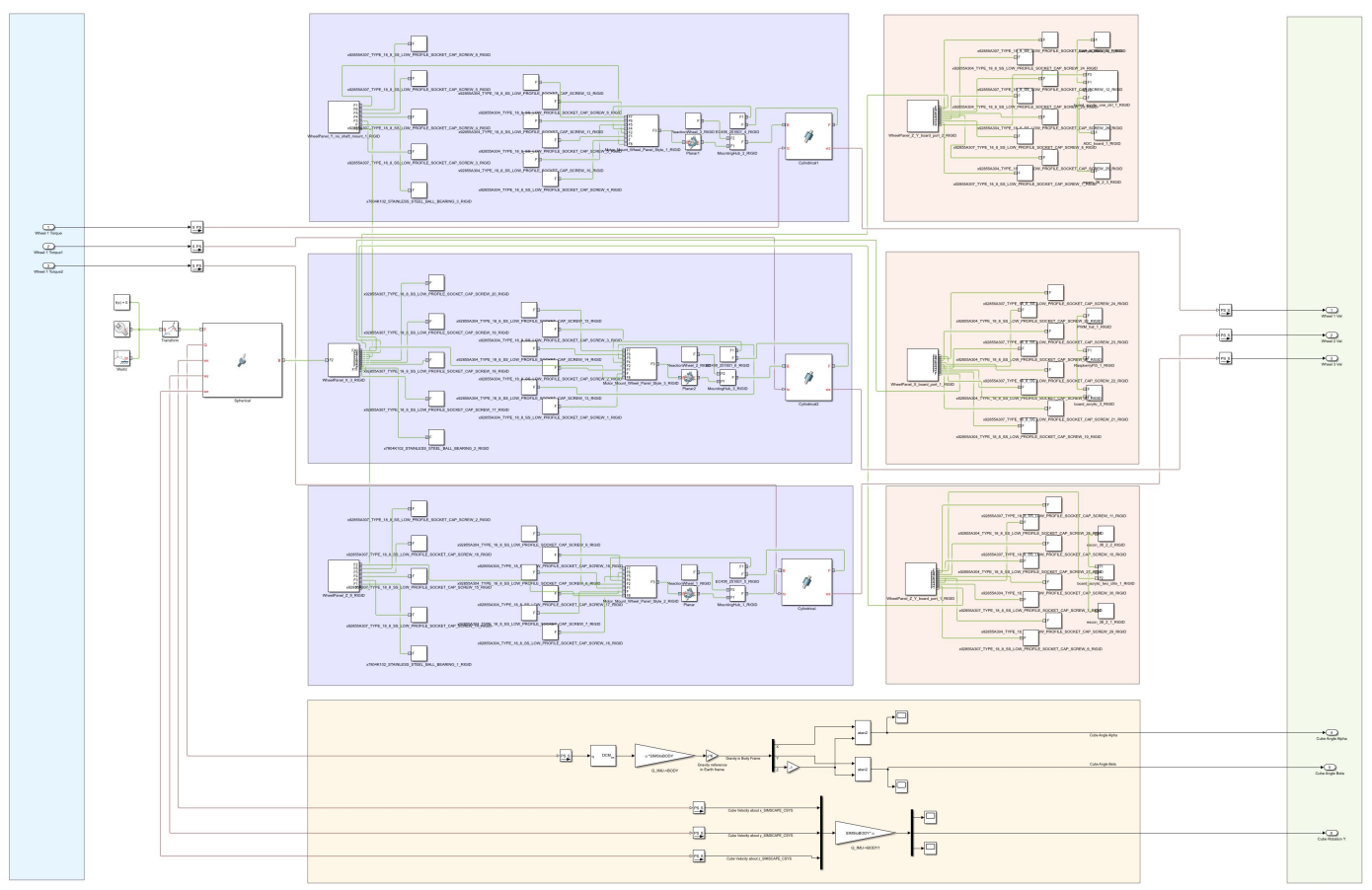

Figure 3.2: Final Simscape model of the cube.

Information flowing into the Simscape model comes in from elements in the cyan rectangle on the left hand side. These torque values are fed to the three cylindrical constraints representing the motors of the system. The outputs of the system, including the attitude of the cube and the rotational velocities of the wheels, flow out of the system through the green box on the right. The yellow area includes the transformation of the cube's orientation and rotational rate measures, as described in Section 3.7.2. A spherical joint is located between the cyan inputs region and the purple areas. A zoomed in view of this portion of the simulation is shown in Figure 
3.3. It describes the cube relative to the reference frame. The spherical constraint block therefore connects to the WORLD block located just to the left of it and the WheelPanel-X-3 block, which is the panel that contacts the ground, to the right.

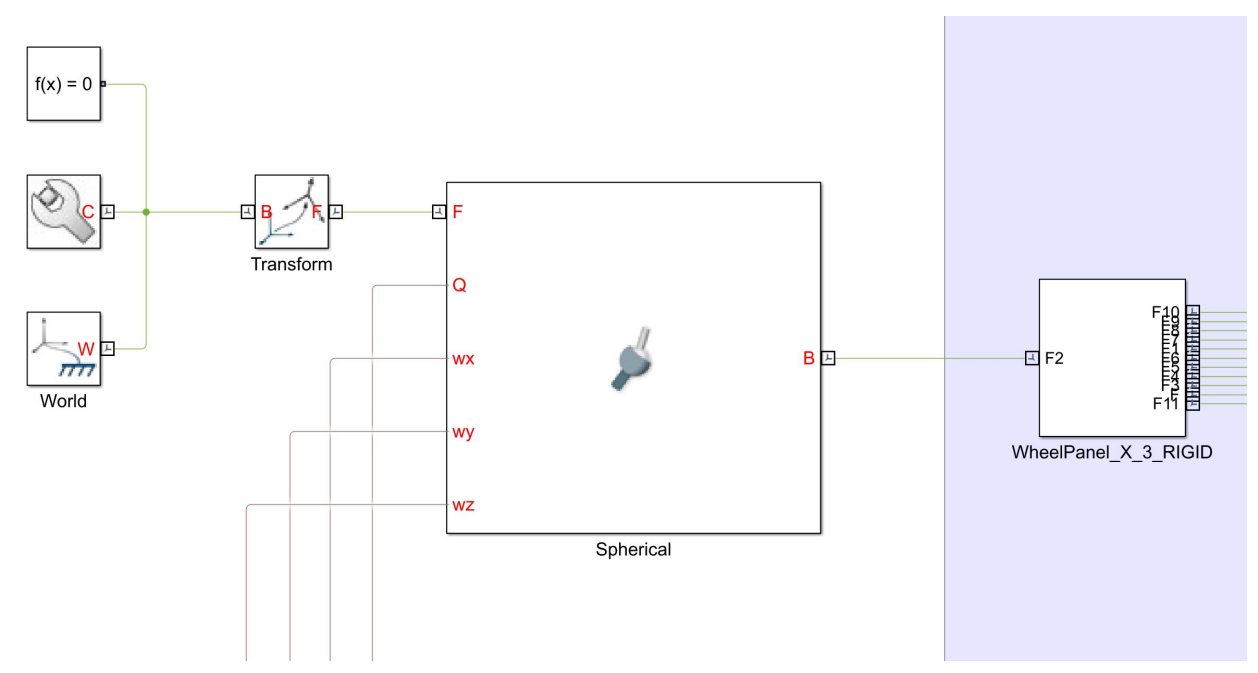

Figure 3.3: Spherical constraint of the simulation. Its base coordinate system is coupled to that of WheelPanel-X-3 while its follower coordinate system connects to the global reference frame of the simulation. Q represents the orientation of the base CSYS relative to the follower CSYS. Wx, $\mathrm{wy}$, and wz are the rotational velocities of the WheelPanel-X-3.

All mechanical parts that are connected to WheelPanel-X-3, including the motor mount, screws, and reaction wheel assembly, are found in the central purple area. The other two purple areas represent the other two wheel panels and their respective components. 


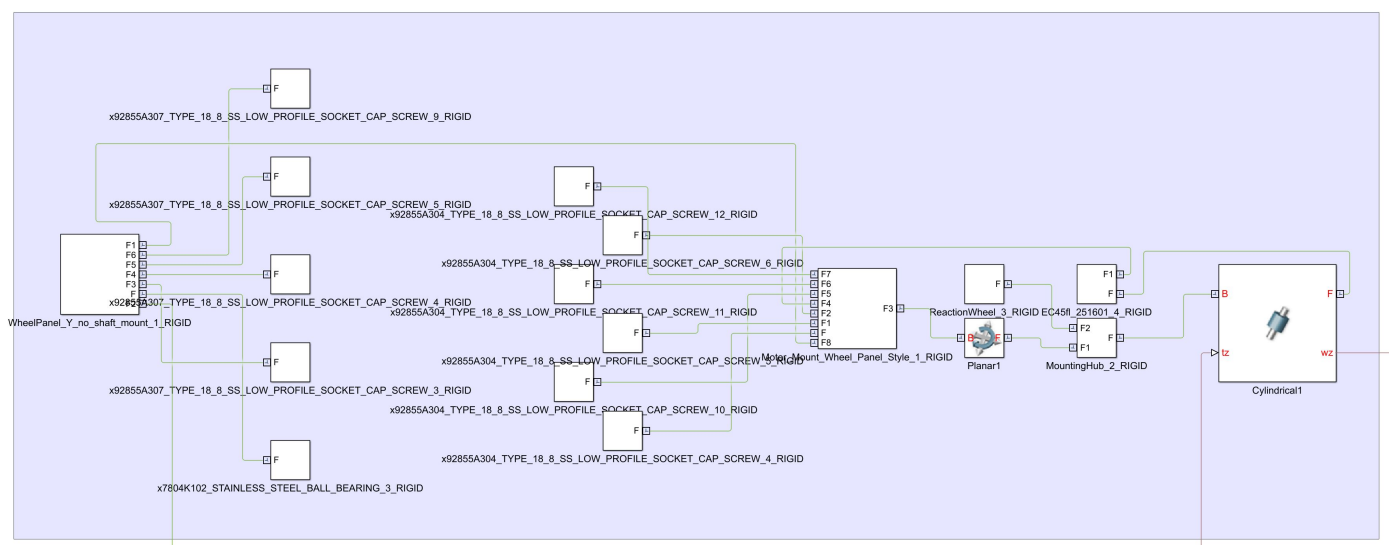

Figure 3.4: Each purple area of the simulation contains the mechanical parts included in a wheel panel assembly, including a panel, a motor mount, the reaction wheel, the mounting hub, and screws.

Just to the right of them are red areas which contain the side panels that hold the electronics and all of the mechanical and electrical parts which are mated to them.

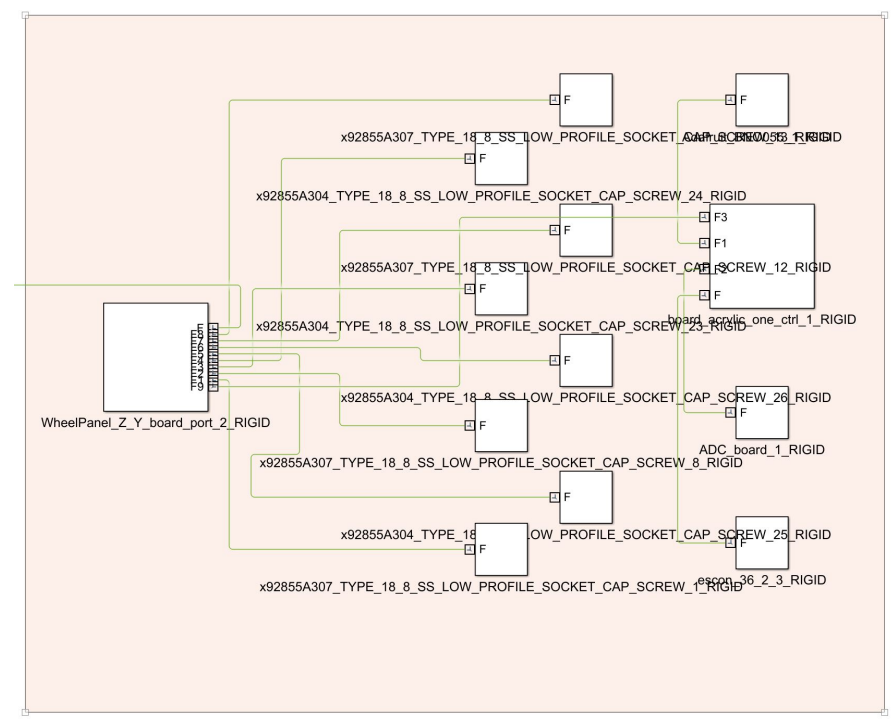

Figure 3.5: The red areas of the simulation contains the blocks representing the mechanical parts which mount to an electronics-bearing side panel. The large square to the left is the aluminum side panel, the one to the right is the acrylic panel, and the other blocks are screws and electronics. 


\subsection{System Constraints}

In order for the simulation to be realistic, the mechanical constraints had to reflect the actual system. The constraints were set in SolidWorks and then transferred to the simulation by way of MathWorks' export tool. The system was mated as follows:

The structural components, including the six panels, three motor mounts, three motor stators, and screws were all fixed relative to the panel which contacts the ground $(+\mathrm{X})$. The electrical boards were fixed relative to their acrylic board panels, which in return were constrained with a fixed mate to their individual side panels. The three motor stators were mated to the motor mounts, again with fixed constraints. Essentially, everything on the cube but the rotating wheels and rotors was defined to act as one solid piece. These mates are represented by lines directly connecting the two blocks representing the parts in SimScape.

For the wheel stacks, the reaction wheels were fixed to the mounting hubs. This connection reflects the real system as the reaction wheels are mated to the mounting hubs with four screws. The mounting hubs were then fixed to the shafts of the motors. The connection between each motor's rotor and stator comprised of a cylindrical joint and a planar joint to define the single degree of freedom of the mechanism.

One minor inaccuracy in the definition of the constraints was that the bearings were modeled as a solid piece fixed to the cube's housing. Therefore, the inertia of the inner race of the bearing and the effective inertia of the balls and cage were not included in the total inertia of the reaction wheel assembly. The inertia of the inner race was estimated to be only about $49 \mathrm{~g}-\mathrm{mm}^{2}$, a mere $0.016 \%$ of the inertia of the reaction wheel assembly. Additionally, the cables were not included in the model. The robust LQR was predicted to be able to handle these minor inaccuracies. 


\subsection{Verification of System Constraints}

The system constraints were easily verified by running the simulation and observing the animation. Several aspects of the dynamics were inspected - none of the parts which should be fixed to each other accelerated directly down, implying that all parts were properly mated to each other, the cube pivoted about the point of contact with three degrees of freedom, and the reaction wheels stayed in place relative to the cube's structure while at the same time having the ability to rotate.

Figure 3.6 depicts the motion of the cube as it spins around the pivot point as calculated by the simulation. The angles $\theta$ and $\phi$ (given by signals 4 and 5 ) increase in a sinusoidal manner until the cube points directly downwards, at which time the Euler angles jump. The three reaction wheels can be observed to accelerate radially due to their inertia and then decelerate again relative to the cube when the mechanism pendulums up.

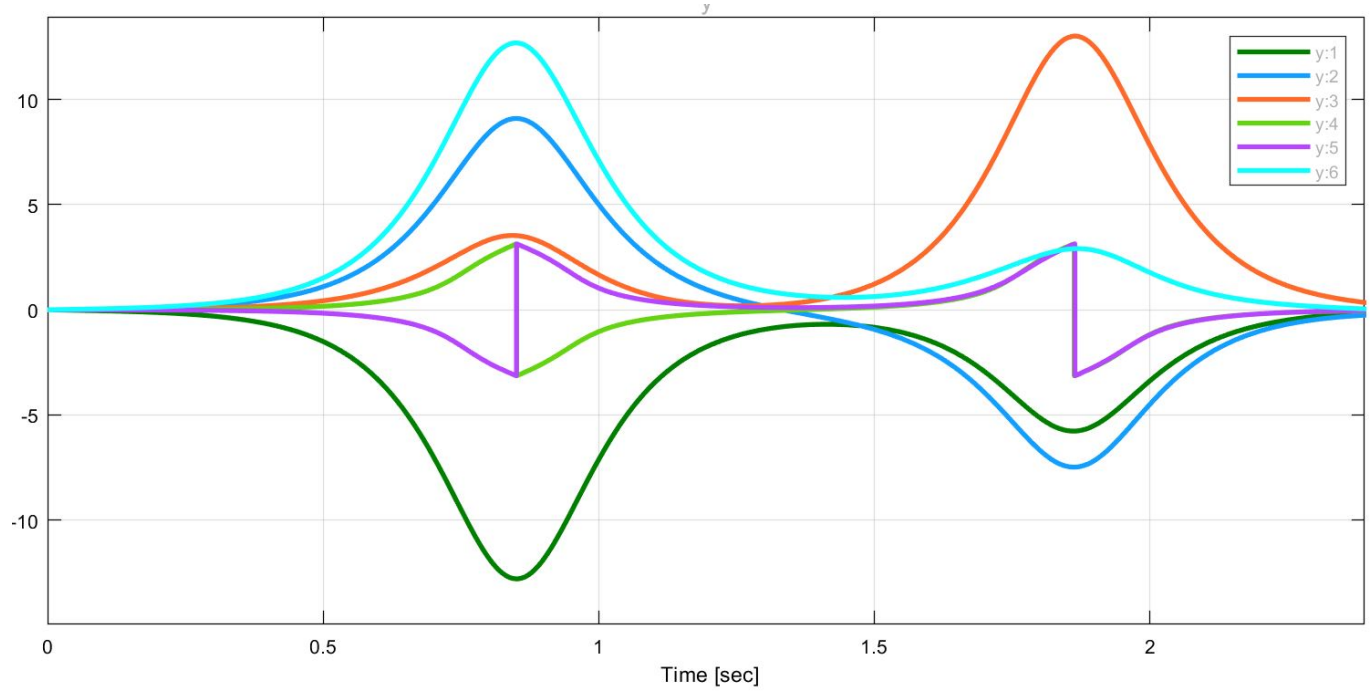

Figure 3.6: Motion of the cube in the simulation with no inputs. 


\subsection{Coordinate Systems}

The simulation uses three coordinate systems to describe the dynamic behavior of the system. These are shown in Figure 3.7 below.

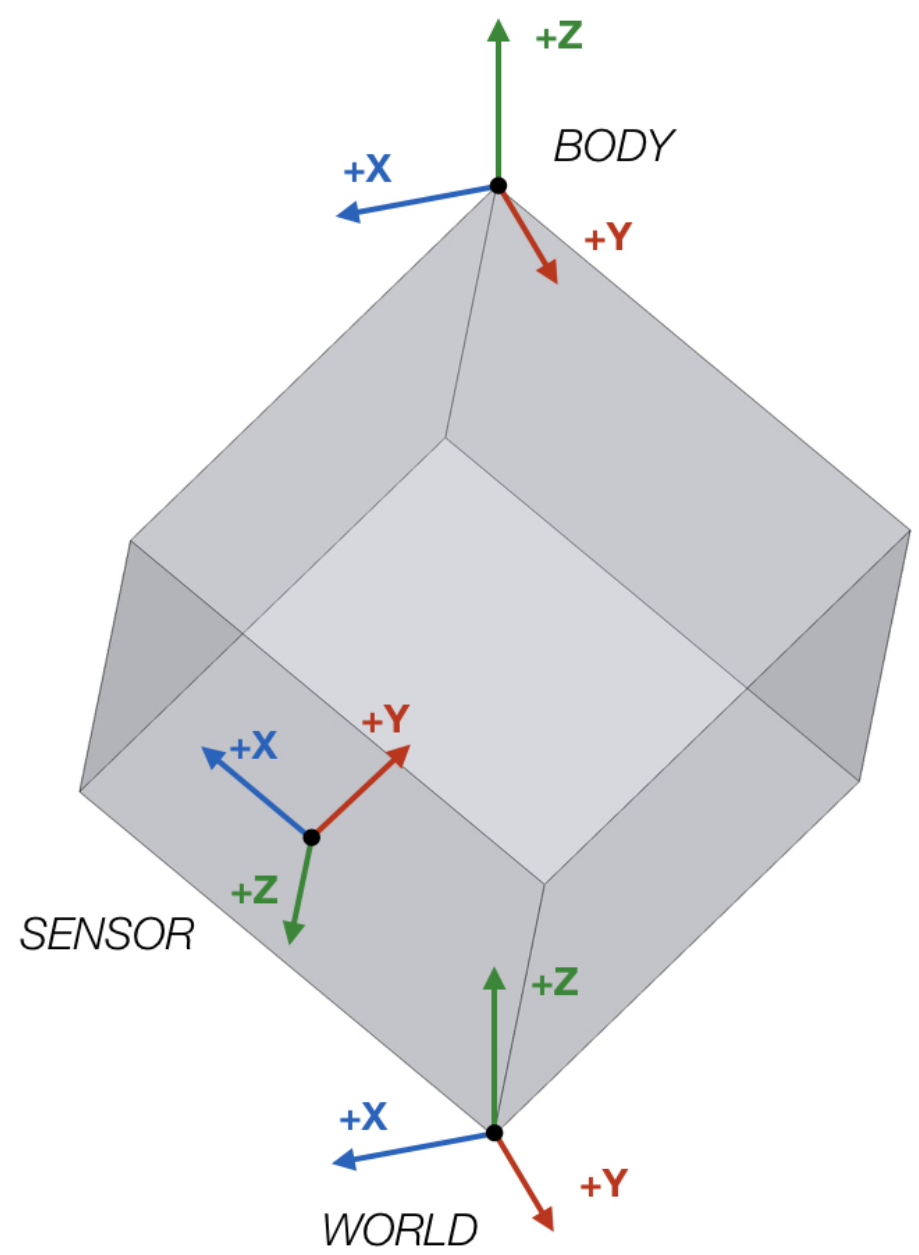

Figure 3.7: Illustration of the cube with its three coordinate systems

$C S Y S_{W o r l d}$ was derived from the CSYS of the main assembly in CAD. It is the reference that the orientation and rotational rates of the cube were measured with respect to. By default, the $-\mathrm{y}$ axis became the direction of gravity. However, to match the sensor's reference frame, the direction of gravity was changed to be in the -z direction. More on this in Section 8.5.1. 
$C S Y S_{\text {Sensor }}$ matches the CSYS of the $+\mathrm{X}$ panel in CAD. SimScape measured the orientation of the cube by comparing $C S Y S_{\text {Sensor }}$ to $C S Y S_{W o r l d}$.

Finally, $C S Y S_{B o d y}$ was defined to have the same orientation as $C S Y S_{W o r l d}$ when the cube is in its upright position (Note that the upright position is that in which the top corner of the cube is directly above the balancing point. The balancing position is that in which the center of gravity is directly above the balancing point). The gravity vector was translated to this CSYS and the desired orientation parameters were calculated from that vector, as described in Section 3.7.2.

\subsection{Tailoring the Model}

\subsubsection{Adding Sensing and Inputs}

To complete the simulation, it was necessary to output the orientation and the rates of rotation of the cube from the simulation. Similarly, the speed of the wheels needed to be measured and the input torque values needed to be fed to the reaction wheels. The elements of the Simscape simulation responsible for defining the constraints between various parts and coordinate systems can be configured to have input and output ports. The spherical constraint, which mates the bottom corner of the cube to a fixed point in space, was modified to have four outputs with one signal describing the orientation of the cube as a quaternion and three signals representing the rotational velocities about $C S Y S_{B O D Y}$. Likewise, the cylindrical constraints between the wheels and the cube were configured to have both an input and an output. For each constraint, the input is the value of the torque which acts on the reaction wheels with equal and opposite torque on the cube. The output signal carries information about the rotational velocity of the wheel.

Lastly, Simulink to PS (for Physical System) and PS to Simulink conversion boxes 
were added to transform between the two different signal types [4]. Therefore, a Simulink type signal could enter the simulation, be converted to the PS signal type, and then define the torque of the motors. On the outgoing side, the orientation of the cube and the velocities of the wheels were sensed in the PS signal type and converted so that the standard Simulink blockset may be used in conjunction. Simscape uses these conversion blocks to make sure that the units of the simulation are correct. In the blocks, the units of the signal were declared. For the torque inputs, Newton-meters were chosen. The angles of the cube were set to be in radians. The blocks converting the rates of the cube and the wheels from the physical model to the Simulink signal type were configured to be in radians/second.

\subsubsection{Modification of the Outputs}

By default, the orientation sensor of the spherical constraint outputs a quaternion which describes the cube's orientation, that is the rotation of $C S Y S_{B O D Y}$ with respect to $C S Y S_{W O R L D}$. As described in Section 4.1, the desired output of the simulation consists of the two angles describing how much the cube leans forwards and backwards and left and right.

To achieve this output, a unit gravity vector $\left(\mathrm{x}=0, \mathrm{y}=0, \mathrm{z}=-1\right.$ in $\left.C S Y S_{W O R L D}\right)$ was first translated to $C S Y S_{S E N S O R}$ using a cosine direction matrix $Q_{W O R L D->S E N S O R}$. This 3x3 matrix was created by converting the quaternion orientation measurement to a cosine direction matrix using the appropriate Simulink block. The resulting vector was then translated to $C S Y S_{B O D Y}$ by multiplying it by the $3 \times 3$ transformation matrix $Q_{S E N S O R->B O D Y}$ representing $C S Y S_{S E N S O R}$ with respect to $C S Y S_{B O D Y}$. This matrix was calculated using the rotation matrix formula for a YZX-sequence transformation as presented in [5]. 


$$
Y_{1} Z_{2} X_{3}=\left[\begin{array}{ccc}
c_{1} c_{2} & s_{1} s_{3}-c_{1} c_{3} s_{2} & c_{3} s_{1}+c_{1} s_{2} s_{3} \\
s_{2} & c_{2} c_{3} & -c_{2} s_{3} \\
-c_{2} s_{1} & c_{1} s_{3}+c_{3} s_{1} s_{2} & c_{1} c_{3}-s_{1} s_{2} s_{3}
\end{array}\right]
$$

The angles of rotation were found using the CAD software's measurement tool. Intermediate CSYS were created and the angle of rotation of each of them relative to the previous one was measured and used in the transformation matrix. The Matlab code to calculate this cosine direction matrix is shown in Appendix A.

By multiplying the unit gravity vector $r_{g, W O R L D}$ through two coordinate transformation $Q_{W O R L D->S E N S O R}$ and $Q_{S E N S O R->B O D Y}$ to $C S Y S_{B O D Y}$, the resulting vector $r_{g, B O D Y}$ points in the same direction but is represented in $C S Y S_{B O D Y}$. This mathematical operation is shown in Figure 3.8. When the cube is in its upright position this vector points in the $-\mathrm{z}$ direction. However as the cube tilts, $C S Y S_{B O D Y}$ rotates as well and as a result $r_{g, B O D Y}$ begins to have non-zero $\mathrm{x}$ and $\mathrm{y}$ values and values of less than 1 in the $\mathrm{z}$ axis.

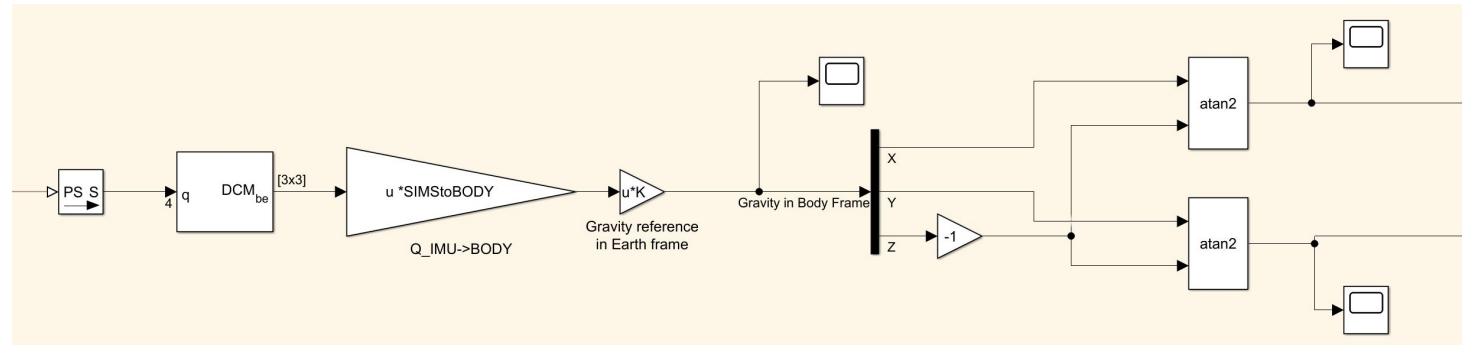

Figure 3.8: Portion of the model responsible for finding the cube's angles $\theta_{\text {cube }}$ and $\phi_{\text {cube }}$ from the quaternion output of the spherical constraint block.

The angles between $r_{g, B O D Y}$ and the $-\mathrm{z}$ axis of $C S Y S_{B O D Y}, \theta_{c u b e}$ and $\phi_{c u b e}$, describe the angle of tilt of the cube in the xz-plane and yz-plane, respectively. Figure 3.9 depicts $r_{g, B O D Y}$, angles $\theta_{\text {cube }}$ and $\phi_{\text {cube }}$ of the cube when it is tilted. 


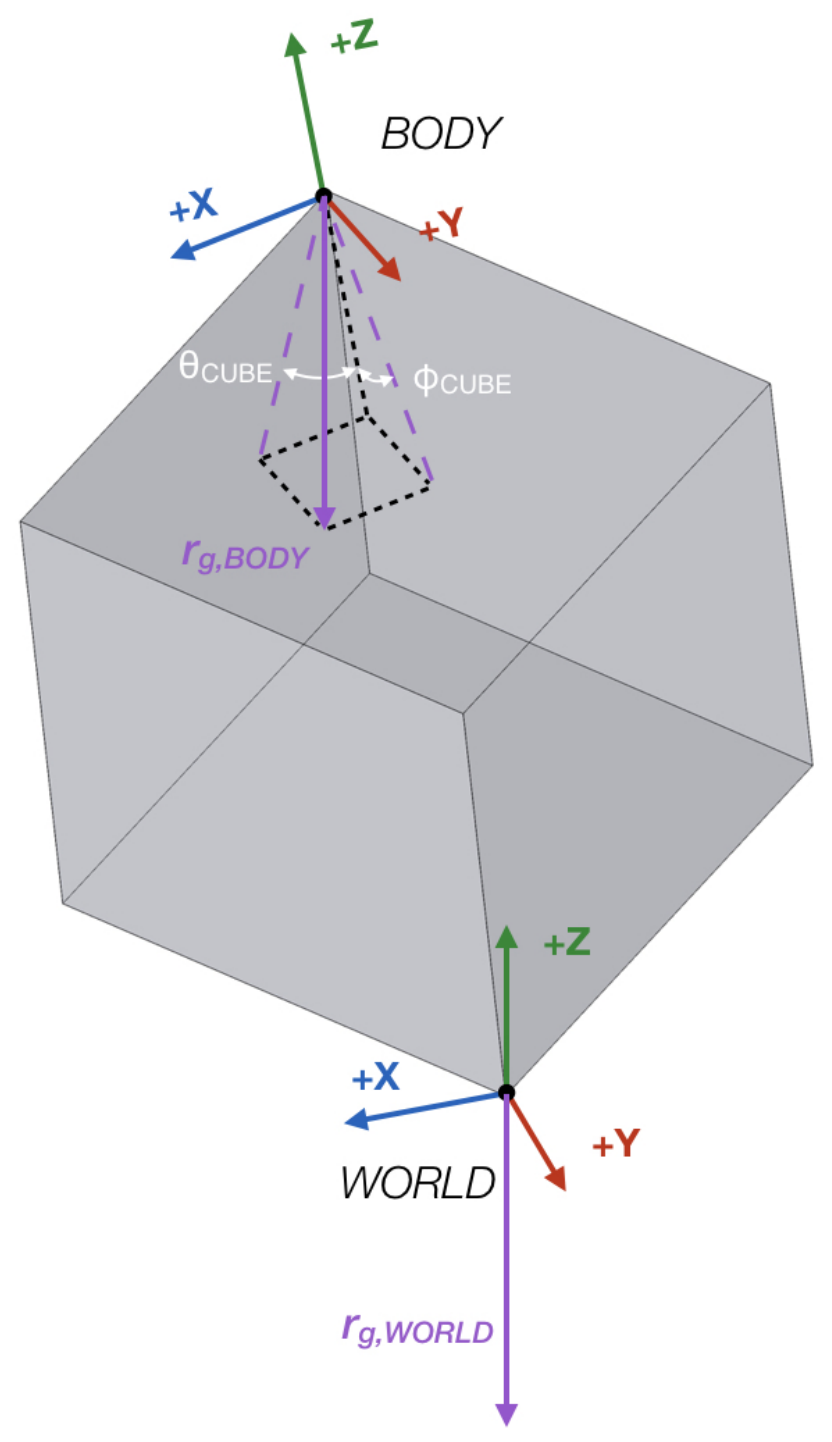

Figure 3.9: Graphical explanation of $\theta_{\text {cube }}, \phi_{c u b e}, r_{g, B O D Y}$, and $r_{g, W O R L D}$.

Another desired output from the simulation was the rotational rate of the cube about the z axis of $C S Y S_{B O D Y}$, since it was deemed important to control this parameter (the reasoning for this decision is outlined in Section 4.1). The simulation outputted, by default, the rotational rates of the cube about the $\mathrm{x}, \mathrm{y}$, and $\mathrm{z}$ axes of $C S Y S_{S E N S O R}$. These values were multiplied by the coordinate transformation matrix $Q_{S E N S O R->B O D Y}$. The resulting vector is the angular rate vector given in $C S Y S_{B O D Y}$. Since only the z-component of this vector was determined to be of in- 
terest, the first two components of this vector were disregarded while the third was passed out of the simulation.

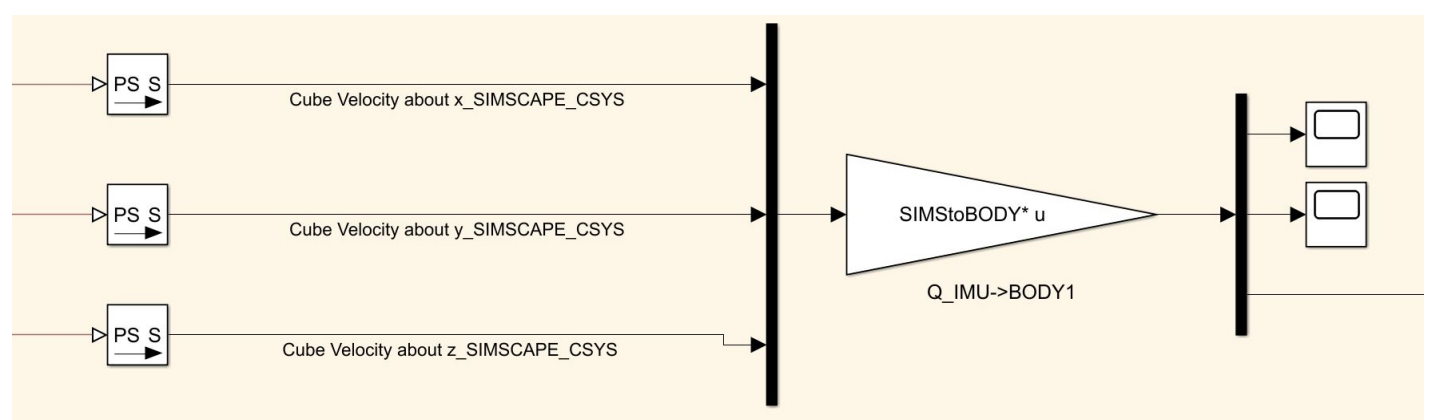

Figure 3.10: Coordinate transformation of angular rates from $C S Y S_{S E N S O R}$ to $C S Y S_{B O D Y}$

Lastly, the wheels' angular rates were outputted from the simulation. These outputs did not require any modification.

\subsection{Linearized System Matrices}

The linearized state space matrices representing the mechanical system were calculated by Matlab using the simulation. Matlab's 'linearize' function steps through the simulation, from the declared inputs to the outputs, linearizing each of the blocks for the initial condition of the system $[6]$. In this case, the system started in the upright position.

Before the system could be linearized, the inputs and outputs had to be declared. These were the same as the inputs and outputs described in Section 3.7.1 and Section 3.7.2. In Simulink, declaring these information lines as inputs and outputs was achieved by right clicking on the lines, selecting linear analysis points, and choosing either 'input' or 'open-loop output'. Figure 3.11 shows the model of the plant (contained in the gray rectangle) with small symbols on the input and output lines, confirming that the signals were properly configured. 


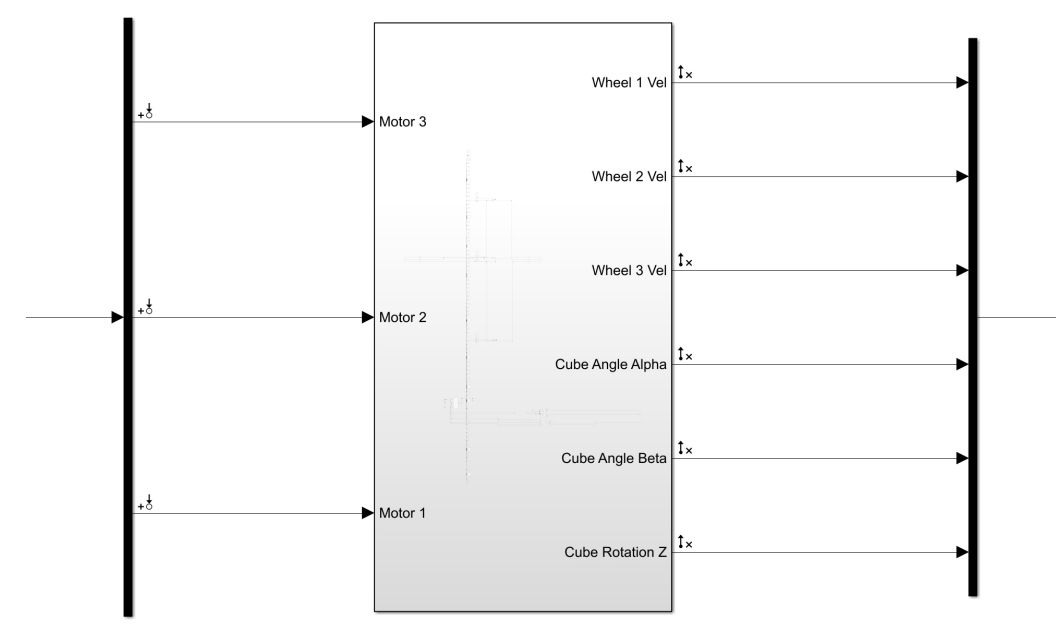

Figure 3.11: The subsystem containing the Simscape model has three inputs which feed the cylindrical constraints and six outputs, three wheel velocities, two cube angles, and one cube rotational velocity. The symbols above the signals represent their declaration as linear analysis points.

It should be noted that a linear system can be described by Equations (3.1), the internal description equations of linear systems $[7]$.

$$
\begin{aligned}
& \dot{x}(t)=A(t) x(t)+B(t) u(t) \\
& y(t)=C(t) x(t)+D(t) u(t)
\end{aligned}
$$

The linearize function took the linear analysis points and the simulation model and returned matrices A, B, C, and D. These four matrices fully describe the dynamic behavior of the system at the point of linearization. Matrix A is a mathematical representation of how the current state causes the state of the mechanism to change. Matrix B outlines how the three inputs cause a change in the state. Matrix C describes the effect of the current state on the output vector. Matrix D contains the relationships between the three inputs and the output vector.

Appendix $\mathrm{A}$ contains the code which was used to linearize the plant. 
Chapter 4

\section{CONTROL ALGORITHM DESIGN}

\subsection{State Space Vector and Controlled Output Vector}

The mechanical system consists of four bodies that can move relative to each other - the body of the cube, which is a combination of the panels, motor mounts, acrylic panels, and electronics, and the three reaction wheel assemblies. Since it pivots about

a point, the cube has three rotational degrees of freedom $\alpha_{c u b e}, \beta_{c u b e}$, and $\gamma_{c u b e}$. Due to the cylindrical constraint of the wheels to the cube, each of the reaction wheel assemblies has one degree of freedom $\theta_{\text {wheelN }}$. The mechanism's state at any given time can therefore be fully described by the state space vector $x$.

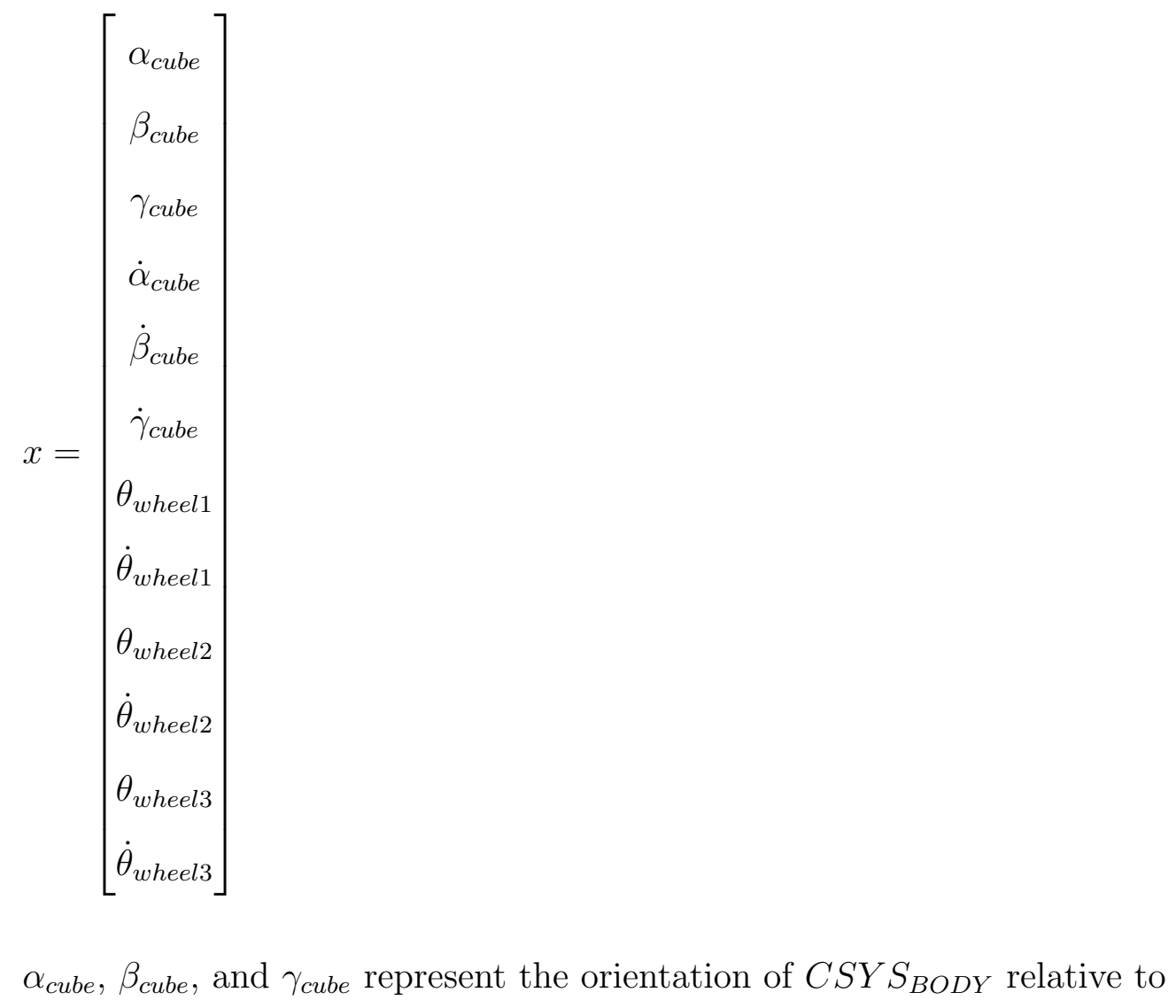


$C S Y S_{W O R L D} . \quad \dot{\alpha}_{\text {cube }}, \dot{\beta}_{\text {cube }}$, and $\dot{\gamma}_{\text {cube }}$ are the rotational velocities of $C S Y S_{B O D Y}$ relative to $C S Y S_{W O R L D}$, and $\theta_{\text {wheelN }}$ and $\dot{\theta}_{\text {wheelN }}$ represent the angles and rotational velocities of the three wheels, respectively.

The controlled output vector $y$ was derived from the state space vector of the system. It describes the parameters of the system which shall be controlled. This vector was chosen to be:

$$
y=\left[\begin{array}{c}
\dot{\theta}_{\text {wheel } 3} \\
\dot{\theta}_{\text {wheel } 2} \\
\dot{\theta}_{\text {wheel } 1} \\
\theta_{\text {cube }} \\
\phi_{\text {cube }} \\
\dot{\psi}_{\text {cube }}
\end{array}\right]
$$

The elements $\dot{\theta}_{\text {wheelN }}$ are the same as in the state space vector, representing the rotational speed of the appropriate wheel N. $\theta_{\text {cube }}$ and $\phi_{\text {cube }}$ represent the angles of the cube about the y and $\mathrm{x}$ axes of $C S Y S_{B O D Y}$, respectively, while $\dot{\psi}_{\text {cube }}$ is the rotational velocity of the cube about the $\mathrm{z}$ axis of $C S Y S_{B O D Y}$.

The driving design decision behind choosing $\theta_{\text {cube }}, \phi_{\text {cube }}$, and $\dot{\psi}_{\text {cube }}$ as elements of the controlled output vector was to not control $\psi_{\text {cube }}$, the angle of the cube about the $\mathrm{z}$ axis of $C S Y S_{B O D Y}$. This was deemed undesirable because controlling this angle would result in the cube rotating about its $\mathrm{z}$ axis when the control algorithm was started. Additionally, any wavering of the IMU's magnetometer readings would result in $C S Y S_{B O D Y}$ to rotate about its own z axis, thus causing the cube to follow the wavering of the magnetometer readings. It was decide that the final orientation of the cube about the $\mathrm{z}$ axis did not matter.

However, it was deemed important to keep the cube from spinning about its 
$\mathrm{z}$ axis. Spinning of the cube would disrupt the visual effect of having an object stable in an unstable position. Furthermore, rotation about the $\mathrm{z}$ axis combined with torques applied at the reaction wheels would results in gyroscopic effects that would significantly increase the complexity of the system dynamics and control algorithm.

To eliminate $\psi_{\text {Cube }}$ from the controlled output vector, the measurement method of the orientation of the cube was modified. Instead of using Euler angles or quaternions to observe the relationship between $C S Y S_{S E N S O R}$ and $C S Y S_{W O R L D}$ at any given time, the angles $\theta_{\text {cube }}, \phi_{\text {cube }}$ and $\psi_{\text {Cube }}$ were derived from the quaternion outputted by the spherical constraint in the simulation. $\psi_{C u b e}$, the angle of $r_{g, B O D Y}$ in the xy-plane, was then excluded in the output vector.

See Section 3.7 .2 for a more thorough explanation of the derivations of $\theta_{\text {cube }}$ and $\phi_{\text {cube }}$ and a visualization of $C S Y S_{W O R L D}, C S Y S_{S E N S O R}$, and $C S Y S_{B O D Y}$.

\subsection{Control Algorithm Architecture}

The goal of the control algorithm was to stabilize the cube in an inherently unstable position with its wheels' speeds reaching steady state conditions. This demanded that the mechanism keep its center of gravity above the point of contact. Doing so would drive any moments about the spherical constraint to zero, making it possible to do achieve steady-state stability.

This balanced position is not the same as the upright position. The upright position was defined as the top corner of the cube being above the bottom corner. Since the machine's center of gravity is not between these two points due to an asymmetrical electrical layout and the inherent imperfections of the actual hardware, the angles $\theta_{\text {cube }}$ and $\phi_{\text {cube }}$ had to be non-zero for steady state operation. However, it was assumed that the balanced position was very close to the upright position and therefore those angles were small and could be controlled by a zero-angle reference. 
By design, $\dot{\psi}_{\text {cube }}$ was determined to be driven to zero to avoid rotation of the cube. Regarding the wheels, it was decided that rotating them slowly would induce less vibrations in the system and make for smoother operation.

Overall, the desired state of the device consists of all elements in the controlled output vector $Y$ going towards zero. Therefore, $y_{\text {ref }}$ was determined to be a $6 \mathrm{x} 1$ matrix of zeros.

A Linear Quadratic Regulator was chosen to control the cube. This controller requires the full state of the system as an input, so a Gaussian state estimator was used in conjunction. The estimator provides an estimate of the full state to the regulator which then determines the inputs $u$ of the system. Below is a depiction of the control architecture. The controlled output vector $y$ is subtracted from the reference vector $y_{r e f}$. The resulting error is fed through the Gaussian estimator which outputs $\hat{x}$, the estimated full state of the system. $\hat{x}$ is then multiplied by the LQR gain which returns $u$, the input vector. These values of $u$ designate the torques of the reaction wheels. The plant responds and its output $y$ changes.

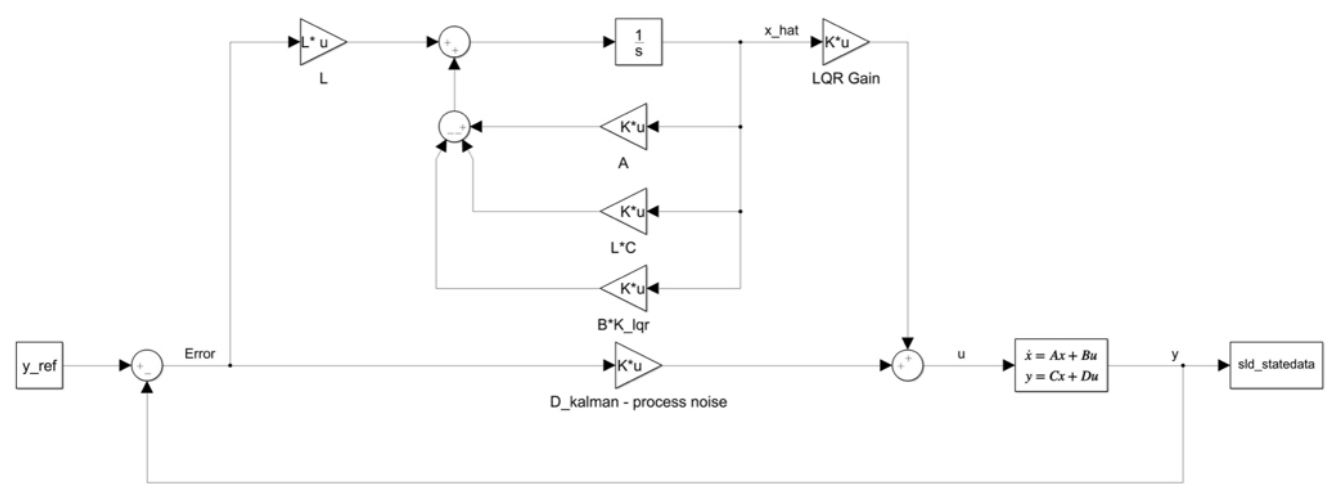

Figure 4.1: Control logic of the cube. The difference between $y_{r e f}$ and $y$ is fed into a state estimator where the full state $x$ is estimated. The estimate $\hat{x}$ is then multiplied by the gain matrix and the result sent to the plant as the motor torques. 


\subsection{Linear Quadratic Regulator}

The LQR is a commonly used controller for linear, multivariable control systems. This algorithm is capable of optimally placing the poles of a coupled system to fulfill a specified goal. It consists of a gain matrix $K_{L Q R}$ which minimizes the quadratic cost function given by Equation 4.1] 8.

$$
J_{L Q R}=\int_{0}^{\infty}\left(\sum_{i=1}^{l} Q_{i i} y_{i}(t)^{2}+\rho \sum_{j=1}^{m} R_{j j} u_{j}(t)^{2}\right) d t
$$

where

$$
\int_{0}^{\infty}\|y(t)\|^{2} d t
$$

represents the energy of the controlled output and

$$
\int_{0}^{\infty}\|u(t)\|^{2} d t
$$

corresponds to the energy of the input signal. $\rho$ is a parameter used to apply more or less weight to the input versus the controlled output. In other words, making $\rho$ large results in a gain matrix $K_{L Q R}$ which minimizes the energy of the input more so than the controlled output, and vise versa. A large value of $\rho$ may result in the total energy applied to accelerating the wheels to be small throughout the motion of the system. On the other hand, a small $\rho$ may result in the integral of the values of each element of the controlled output vector $y$ throughout the motion to be small.

Of course, prioritizing the importance of one energy over another has its trade-offs. A small input energy will likely cost a large amount of output energy and vise versa.

The matrices $Q$ and $R$ scale the elements in the controlled output vector $y$ and input vector $u$. These matrices are very useful for vectors with elements of different 
units. To explain using an example, the cube's output vector $y$ contains state variables pertaining to the angle of the cube, rotational rates of the cube, and wheel velocities. The angle of the cube is expected to be within, say 5 degrees or 0.087 radians of zero. The rotational rate of the cube shall be no more than 10 degrees/second or 0.175 radians/second, while the wheel speeds can be expected to be much faster, say up to $500 \mathrm{rpm}$ or 52.3 radians/second. If $Q$ was an identity matrix, the LQR would surely attempt to minimize the controlled output portion of the cost function for the speed of the wheels since the wheel velocity values were inherently two to three orders of magnitude larger than the cube's orientation or rate of rotation values.

Since controlling the cube's orientation, rates of rotation, and wheels' velocities are of approximately equal importance, Bryson's rule was used to define the matrix $Q:$

$$
Q_{i i}=\frac{1}{\text { maximum acceptable value of } y_{i}^{2}}
$$

The maximum acceptable values of $y$ were determined to be:

$$
\begin{array}{ll}
\dot{\theta}_{\text {wheelN,max }}: & 500 \mathrm{rpm}=52.36 \mathrm{rad} / \mathrm{s} \\
\theta_{\text {cube,max }}: & 5 \mathrm{deg}=0.087 \mathrm{rad} \\
\phi_{\text {cube } \text { max }}: & 5 \mathrm{deg}=0.087 \mathrm{rad} \\
\dot{\psi}_{\text {cube,max }}: & 10 \mathrm{deg} / \mathrm{s}=0.175 \mathrm{rad}
\end{array}
$$

Therefore, 


$$
\begin{aligned}
& {\left[\begin{array}{ll}
\frac{1}{\left(\dot{\theta}_{\text {wheel } 3, \text { max }}\right)^{2}} & \\
& \frac{1}{\left(\dot{\theta}_{\text {wheel } 2, \text { max }}\right)^{2}}
\end{array}\right.} \\
& Q_{i i}= \\
& \frac{1}{\left(\dot{\theta}_{\text {wheel1,max }}\right)^{2}} \\
& \frac{1}{\left(\theta_{\text {cube }, \text { max }}\right)^{2}} \\
& \frac{1}{\left(\phi_{\text {cube, } \max }\right)^{2}} \\
& \left.\frac{1}{\left(\dot{\psi}_{\text {cube } \max }\right)^{2}}\right]
\end{aligned}
$$

Similarly, Bryson's rule was also used to determine $R$, the matrix which scales the input elements relative to each other.

$$
R_{j j}=\frac{1}{\text { maximum acceptable value of } u_{i}^{2}}
$$

The maximum value for any of the wheel speed was set to

$$
T_{\max }: \quad 0.5 \mathrm{~N}-\mathrm{m}
$$

Therefore,

$$
R_{j j}=\left[\begin{array}{ccc}
\frac{1}{\left(T_{\max }\right)^{2}} & & \\
& \frac{1}{\left(T_{\max }\right)^{2}} & \\
& & \frac{1}{\left(T_{\max }\right)^{2}}
\end{array}\right]
$$

The Matlab function $l q r$ takes the state space system, the $Q$ matrix, the $R$ matrix, and the $N$ matrix as arguments and returns the LQR gain matrix. It minimizes the following cost function given by Equation (4.6) [9].

$$
J_{L Q R}=\int_{0}^{\infty}\left(x^{T} Q x+u^{T} R u+2 x^{T} N u\right) d t
$$

The last element of the equation can be utilized to minimize the energy cost for 
the product of the input and output vectors. This was not required for the control of the cube and therefore $\mathrm{N}$ was chosen to be zero. Note that in this equation, $x$ stands for the output vector.

Matlab solved the quadratic equation by finding the solution $\mathrm{S}$ of the algebraic Ricatti equation given by equation 4.7.

$$
A^{T} S+S A-(S B+N) R^{-} 1\left(B^{T} S+N^{T}\right)+Q=0
$$

Appendix A contains the Matlab code used to find the LQR gain. Note that matrices $Q, R$, and $N$ were labeled $Q Q_{l q r}, R R_{l q r}$, and $N N_{l q r}$ in the Matlab code.

\subsection{State Estimator}

The feedback loop of the simulation carries information which partially describes the state of the mechanism. The LQR gain, however, requires the full state space representation as an input. A Gaussian estimator was developed to bridge this gap by estimating the full state of the system.

The state estimator assumes a continuous plant model given by Equations (4.8) 10 .

$$
\begin{array}{r}
\dot{x}=A x+B u+B_{w} w \\
y=C x+v
\end{array}
$$

$w$ represents the process noise and models uncertainty in the system model. $v$ is the sensor noise and therefore models uncertainty in the measurement. The equations of the estimator are given in Equations (4.9) [10]. 


$$
\begin{array}{r}
\dot{\hat{x}}=A \hat{x}+B u+L(y-\hat{y}) \\
\hat{y}=C \hat{x}+v
\end{array}
$$

The gain matrix L is the solution to the algebraic Ricatti Equation (4.10] [11.

$$
L=\left(P C^{T}+\bar{N}\right) \bar{R}^{-} 1
$$

where $\bar{R}$ and $\bar{N}$ are defined as

$$
\begin{array}{r}
\bar{R}=R+H N+N^{T} H^{T}+H Q H^{T} \\
\bar{N}=G\left(Q H^{T}+N\right)
\end{array}
$$

Matlab calculated the estimator gains using the kalman function. The function takes inputs $Q n, R n, N n$ and the parameters of the system and returns the kalman gain $K_{\text {kalman }}$ [1]. These inputs were defined as

$$
\begin{gathered}
Q n=E\left(w w^{T}\right) \\
R n=E\left(v v^{T}\right) \\
N n=E\left(w v^{T}\right)
\end{gathered}
$$

The matrices $Q n, R n, N n$ are the covariance matrices associated with the noise of the system. $w$ represents the white process noise while $v$ is the white measurement noise. The process noise in the mechanism is the noise of the input signal. Karl Johan Åström represented the process and measurement noise sources in block diagram form as shown in Figure 4.2 [12]. 


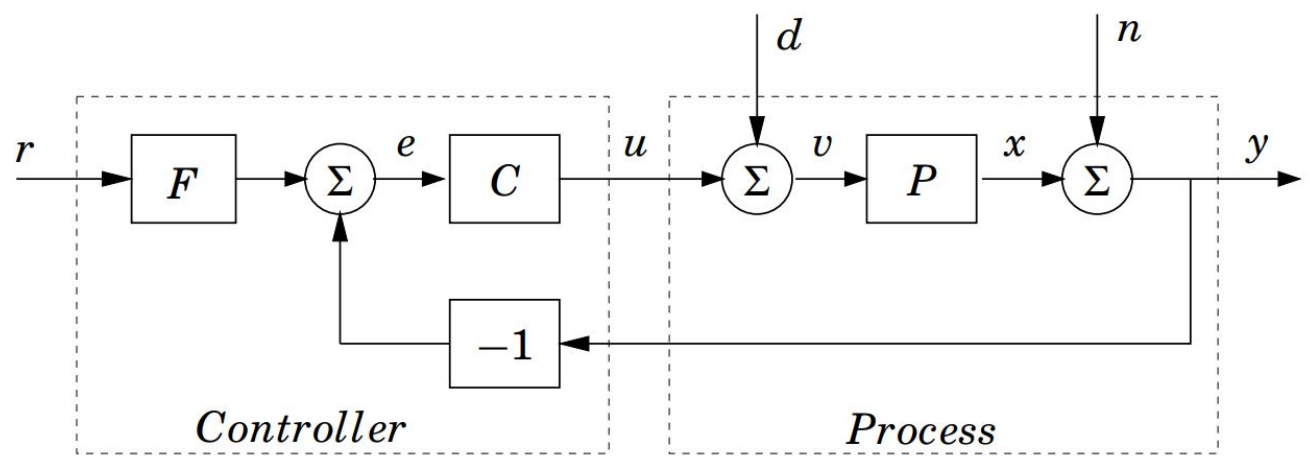

Figure 4.2: Modeling of noise in control systems.

The covariance matrix, according to Nathaniel Helwig is defined as

$$
S=\left[\begin{array}{ccccc}
s_{1}^{2} & s_{12} & s_{13} & \ldots & s_{1 p} \\
s_{21} & s_{2}^{2} & s_{23} & \ldots & s_{2 p} \\
s_{31} & s_{32} & s_{3}^{2} & \ldots & s_{3 p} \\
\ldots & \ldots & \ldots & \ldots & \ldots \\
s_{p 1} & s_{p 2} & s_{p 3} & \ldots & s_{p}^{2}
\end{array}\right]
$$

where $s_{j}^{2}$ is the variance of the $\mathrm{j}$-th variable and $s_{j k}$ is the covariane between the $\mathrm{j}$-th and k-th variables $[13]$.

The covariance matrix of the measurement noise $R n$ was populated with diagonal terms only, meaning that the errors of the measurements of the output vector elements were assumed not to affect each other. The table below lists the predicted values of the variances.

$$
\begin{aligned}
& \sigma_{\text {WheelSpeed }}=1[\mathrm{rpm}] \\
& \sigma_{\text {CubeAngle }}=1[\mathrm{deg}] \\
& \sigma_{\text {CubeRate }}=3[\mathrm{deg} / \mathrm{s}]
\end{aligned}
$$

Therefore, 


$$
R n=\left[\begin{array}{cccccc}
\sigma_{W S}^{2} & & & & & \\
& \sigma_{W S}^{2} & & & & \\
& & \sigma_{W S}^{2} & & & \\
& & \sigma_{C A}^{2} & & \\
& & & \sigma_{C A}^{2} & \\
& & & & \sigma_{C R}^{2}
\end{array}\right]
$$

It would not be accurate to populate the covariance matrix of the process noise Qn similarly to how $R n$ was developed, since the values are amplified by the LQR gain matrix. Instead, $Q n$ was defined as

$$
Q n=I
$$

The matrix $N n$ was set to zero since no effect of the process noise on the output noise was predicted to occur.

\subsection{Implementation of the Control Algorithm into the Simulation}

The next step was to implement the LQR gain and state estimator into the simulation. The Simulink model shown in Figure 4.1 was constructed around the Simscape model (Note that the figure shows a state space block instead of the SimScape model). 


\section{MECHANICAL DESIGN}

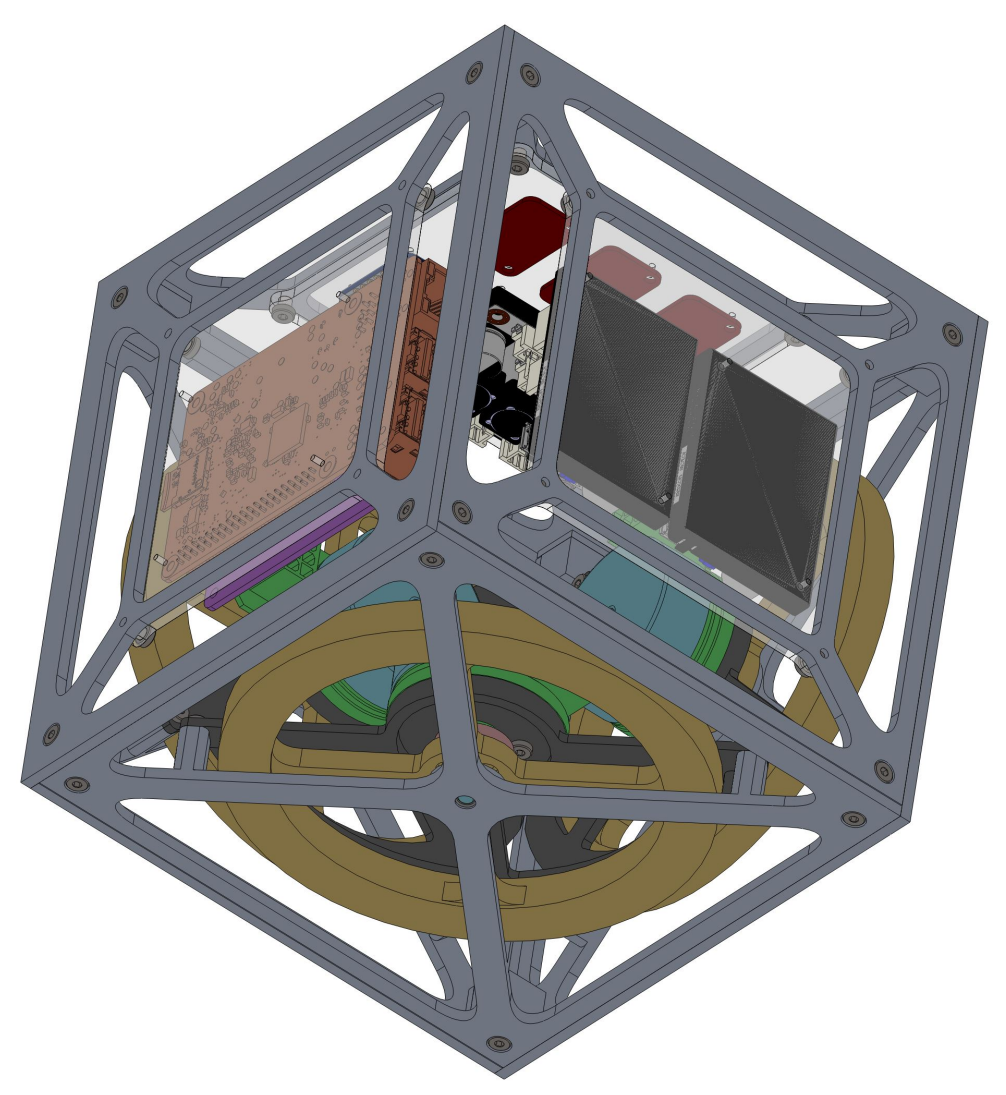

Figure 5.1: CAD model of the cube. The yellow components are the reaction wheels, the motor mounts are dark gray, the stator and rotor of the motors are green and torquoise, the Raspberry Pi is orange, and the motor drivers are shown in black.

\subsection{System Architecture}

The mechanical hardware consists of three reaction wheels which are orthogonal to each other and live within an aluminum structure. They lie relatively close to the external panels of the cube in order to maximize their diameter and therefore inertia while keeping the cube as small and light as possible. This decision drove the overall 
architecture; the motors were placed inside of the reaction wheels with their shafts pointing outward. To fix the motors within the structure, they were attached to motor mounts which bolt onto each of the wheel panels. As can be seen in Figure 5.15 , the reaction wheels are sandwiched between the outer panel and motor mount.

It was decided early on that the cube shall only balance on a specific corner to make it as easy as possible for the reaction wheels to balance the cube. It was found that keeping the center of gravity of the mechanism low would result in a small overall torque required to right the cube up to its balanced point. Since the reaction wheels, motor mounts, and motors are the heaviest asymmetrical objects of the assembly, the corner which the cube was designed to balance was found to be the vertex of the panels that hold reaction wheels.

The size of the cube was chosen with a few factors in mind. There exists a lower limit which is defined by the geometry of the motors, as shown in Figure 5.2 .

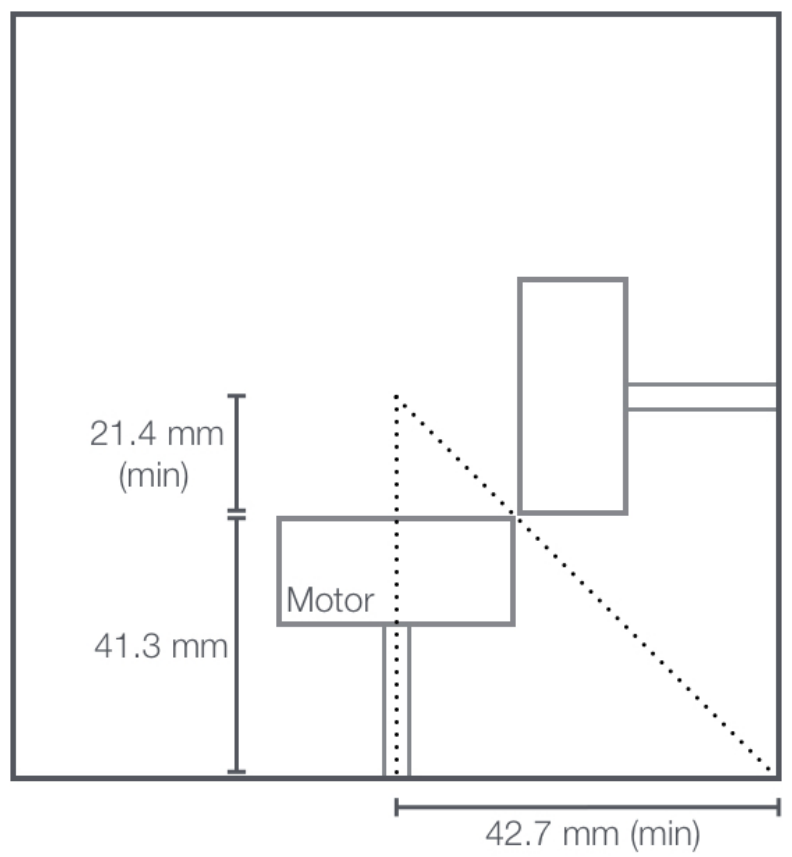

Figure 5.2: Explanation of minimum cube size given the usage of EC45 motors. 
Another limit exists where small off-the-shelf batteries fit into the desired spaces between the motors. Beyond these limits, a larger cube was estimated to result in a better inertia to mass ratio of the reaction wheels and therefore be favorable for the system dynamics. However, a larger size would result in more mass and therefore require higher power motors and electronics.

Borrowing from the design of the Cubli, the cube was designed to have $15 \mathrm{~cm}$ sides.

The structure is composed of six panels and three motor mounts. Two of the panels, called the master panels, sandwich the other four side panels as described in Section 5.2.1. The lower three panels contain the reaction wheels while the upper three panels house acrylic panels which the electrical boards mount to, as described in Section 5.2.6.

\section{$5.2 \quad$ Structure}

\subsubsection{Panel Layout}

The structure of the cube consists of six aluminum panels. These panels have to extend to each of the eight corners and twelve edges but may not intersect. A few different layouts were brainstormed in order to develop a structure which adhered to these design constraints.

One layout consisted of panels which lived in the same envelope. The edges of the panels would be at 45 degree angles to the outer surface of the cube. This design allowed for the panels to be very similar in that each would have a footprint of 15 $\mathrm{cm} \times 15 \mathrm{~cm}$. The downside of this design was that the edges of these panels would be infinitely sharp in theory but in reality these sharp edges would be rounded off by the manufacturing process, handling, and assembly. Therefore, the cubes edges and 
corners would not be sharp which was determined to be undesirable. Another issue with this design was the datum architecture. Four of the six panels would only be attached to one datum. They would have three degrees of freedom; two translational degrees and one rotational.

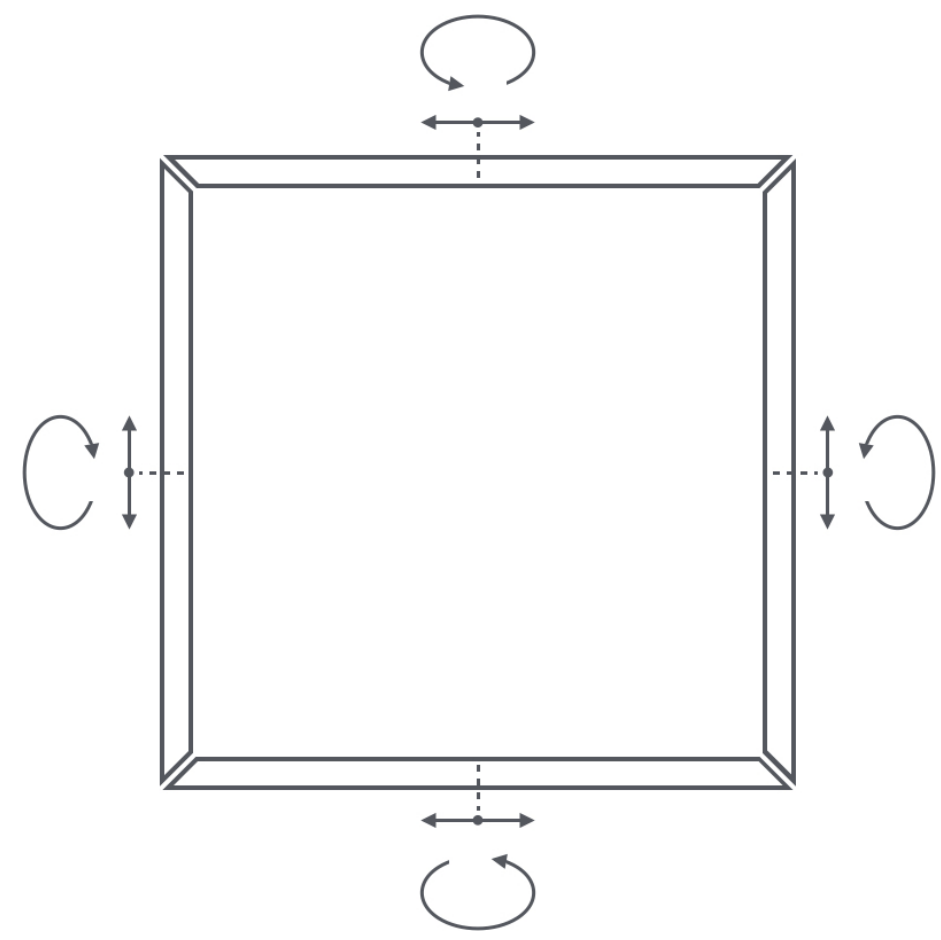

Figure 5.3: Side panels are datum-less if the edges are cut to 45 degree angles.

The second layout called for four panels that surround the cube and two panels which act as lids. The four main panels would have a height of $15 \mathrm{~cm}$, rising from the bottom surface of the cube to the top. Each of them would also extend to one side of the cube and leave space on the opposite side for the neighboring panel. Then, to finish the layout, two lids would be square shaped and extend to the insides of the four surrounding panels.

Yet another layout was inspired by the idea of making the three lower panels which hold the reaction wheels to be the same. However, it quickly became apparent that this was not possible. Each of the panels would extend to one of the edges connected 
to the bottom vertex. The bottom vertex would not be reached by any of the panels due to interference and there would be a cube shaped lack of material at the bottom of the cube.

The final layout is most similar to the second layout. However, instead of there being two lids which dont touch any of the outside surfaces, the top and bottom panels, referred to as the 'master' panels, extend to all four surfaces. The interior surface of these two panels are a datum features for the other four panels which are referred to as 'minor' panels.

The next step was to choose between two different layouts for the minor panels. The first option consisted of two panels which span the full width and two which fit in between, as illustrated in Figure 5.4 .

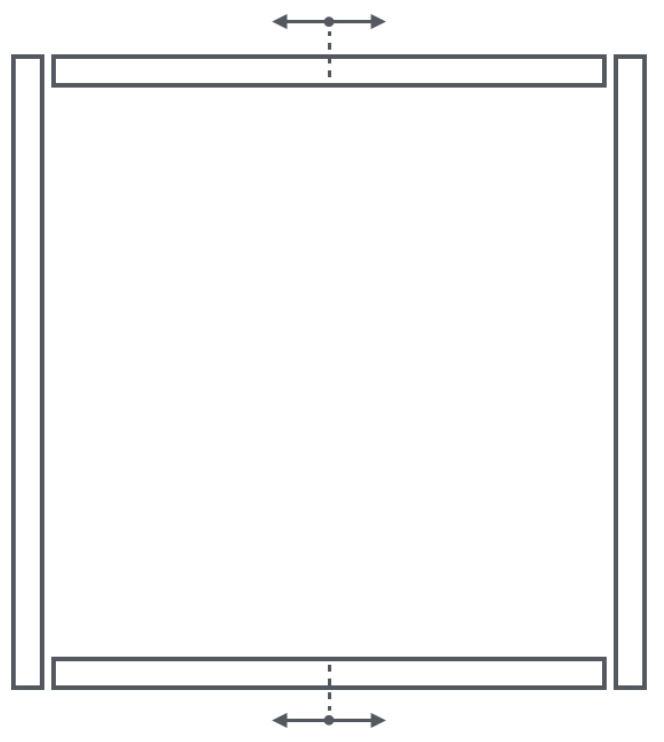

Figure 5.4: Two side panels are datum-less if they do not extend to the edges.

The second option consisted of four panels, each of which contacts one outer surface of the cube as shown in Figure 5.5 . 


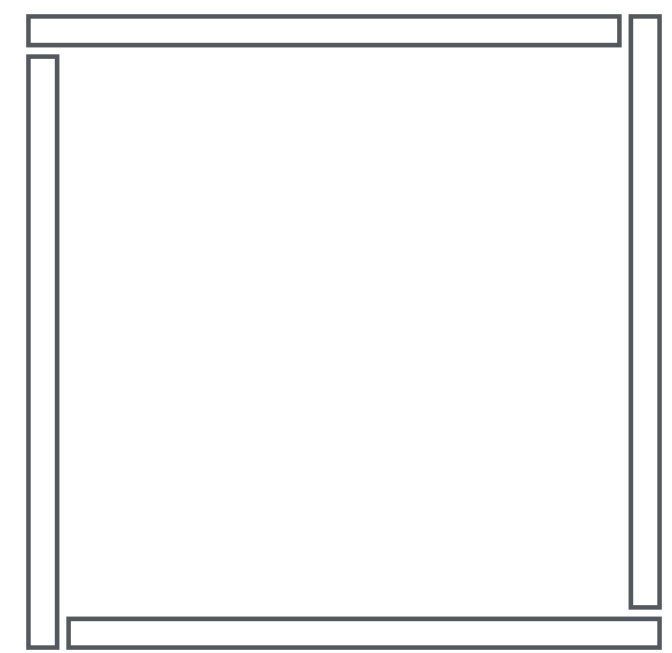

Figure 5.5: Final side panel layout for the cube

The latter option was deemed to be superior for two reasons. First, the four panels are symmetric in size which significantly simplified the fixturing process during the machining phase. Second, the former option would have resulted in uneven gaps in the structure since the small panels would not have a locating feature in one direction.

\subsubsection{Joints}

The next detail to be worked out concerned the method of joining the six panels together. There needed to be extra material somewhere in the structure since the screws did not fit into the thickness of the panels. Two options were considered adding separate components that panels would be screwed to or adding material to some of the panels.

Various designs originated during the brainstorm for adding components. The first consisted of L-shaped rails lining the inside of the edges of the cube. The panels would then be screwed to these rails. Another option was to add cube shaped blocks to the inside of the corners. A brief and unfruitful online search ensued for off-theshelf parts that could be used for both of these options. The small size of the L-shaped 
brackets made it difficult to find anything worthy while no standard blocks were found that would fit the corners well. At the end, the major arguments against using extra components were the lack of good datums and the additional manufacturing effort. Regarding the resulting datum structure, adding a handful of parts would inevitable cause non-zero angles and offsets between the blocks and therefore cause the cube to be distorted. An exaggerated illustration is depicted in Figure 5.6.

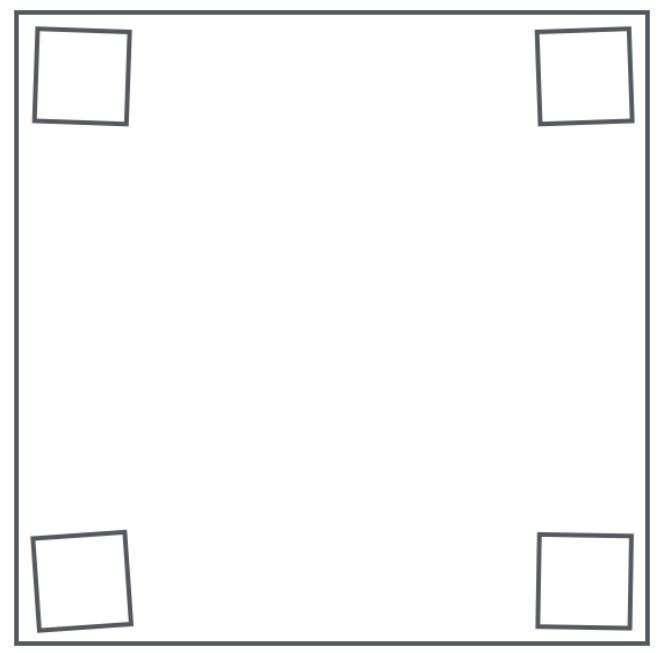

Figure 5.6: Expected results of using seperate blocks for the corners.

For the reasons outlined above, extrusions were added to the master panels. The size of these cubes was defined by the distance of the screws away from the edges and their length. It was advantageous to keep these cubes small, both for aesthetic appeal as well as stock thickness and therefore price. However, they had to be large enough to allow for the screws to have a few threads of engagement. Making these extrusions cubes was a purely aesthetic decision.

Two types of screws were considered to mate the side panels to blocks on the master panels. Either socket head screws or flat head screws would create a relatively continuous surface with the cube's outer surfaces. The decision was made to use low profile socket head screws. Unlike flat head screws, they do not cause the holes to 
align and cause over-constraint issues in the design. Low profile screws were chosen for their small head height. Despite their low profiles, making the screws flush with the outer surfaces of the cube demanded that material be added to the inside of the side panels.

\subsubsection{Datum Structure}

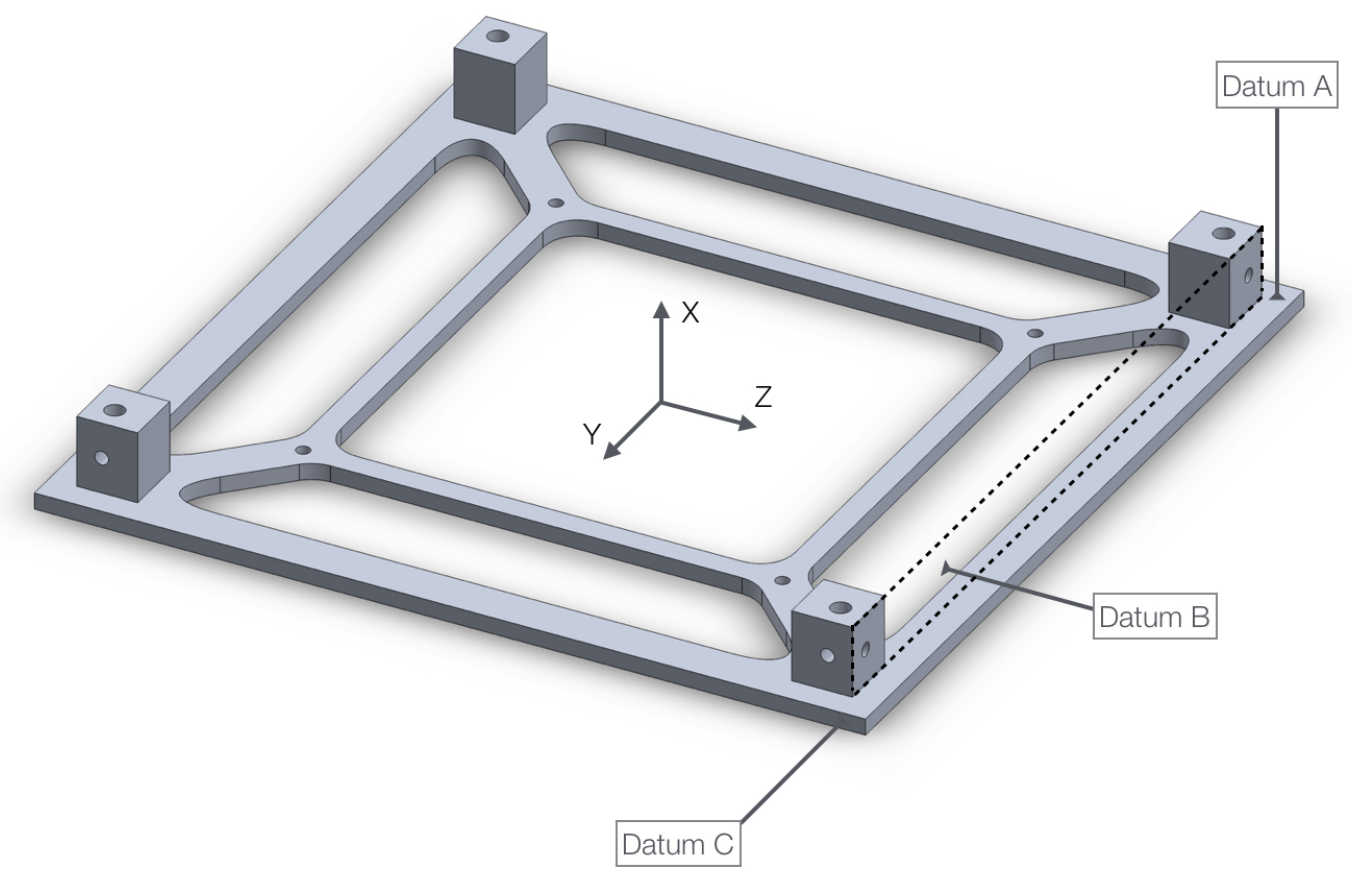

Figure 5.7: Datum structure on a master panel

The master panels contain all of the datums necessary to fully constrain each of the side panels. The side panels are restricted in the $\mathrm{x}$ direction by the interior surface of one of the master panels, labeled Datum A in Figure 5.7. For the panel which attaches onto the $+z$ side of the master panel, it's location in the $z$ direction is determined by two blocks, labeled Datum B. Finally, the panel's y location is fixed by making its outer surface flush with Datum $\mathrm{C}$ of the master panel.

The other three side panels' locations are defined similarly. Datum A works for 
all panels. However, datums $\mathrm{B}$ and $\mathrm{C}$ are unique to the $+\mathrm{z}$ panel. The other three panels use their own set of datums which are identical as datums B and $\mathrm{C}$ but rotated 90, 180, and 270 degrees about the $\mathrm{x}$ axis.

The opposite master panel contains the same datum structure.

\subsubsection{Gaps and Screw Clearance}

In order to avoid over-constraining the system, there have to be gaps between the panels. There is a manufacturing tolerance associated with the size of the panels, so to be able to use both the $\mathrm{C}$ datum of one panel and the $\mathrm{B}$ datum of a neighboring panel there must exist a small gap.

Figure 5.8 shows the nominal gaps designed into the mechanical structure. Note that the horizontal panel may shift to the left and right and still have enough clearance to the vertical panel. Likewise, the vertical panel can shift up and down and also have some room to move.

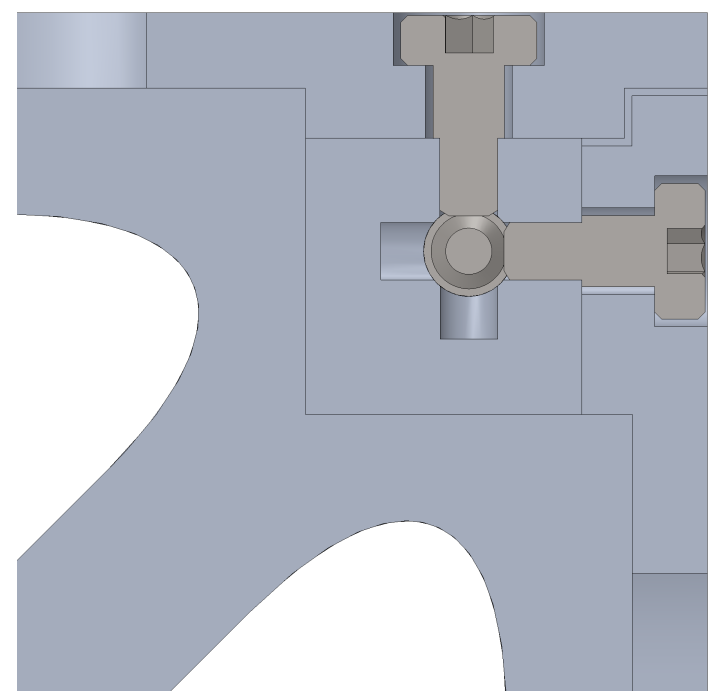

Figure 5.8: Zoomed in screenshot of the gaps between the side panels. 


\subsubsection{Side Panels}

The final wheel-bearing master panel $(+\mathrm{x})$ is depicted in Figure 5.9. The edges are $15 \mathrm{~cm}$ long, spanning the full width and length of the cube structure. Four posts provide mounting surfaces for the four side panels. Each of these have perpendicular holes which the side panels get screwed to. A lot of material was removed from the inside of the panel to reduce weight and to allow for physical and visual access to the interior of the structure. The cross struts of the panels are symmetric about the cylindrical hole at the center of the panel. The rounded corners of the triangular cutouts have a non-constant radius of curvature to give a continuous feel to the edges of the cutout region. The three tower features provide mounting surfaces and holes for the motor mounts, which are described in further detail in Section 5.14 .

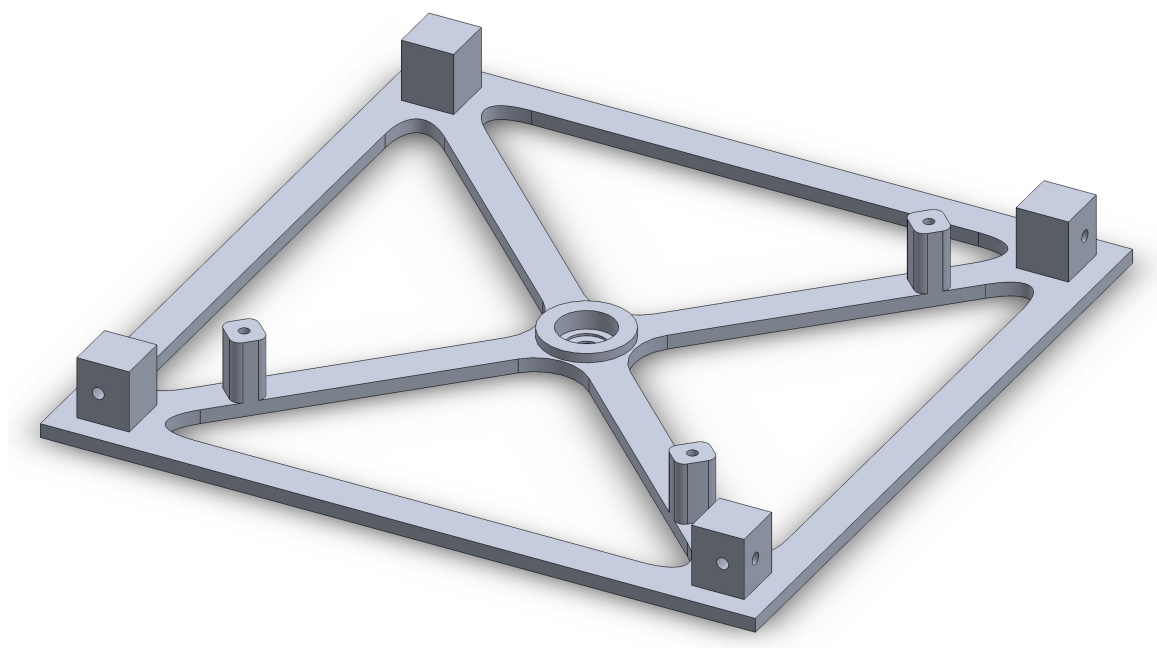

Figure 5.9: Final design of the $+\mathrm{X}$ side panel.

On the opposite side of the cube is the $-\mathrm{x}$ panel which is displayed in Figure 5.10 . This master panel is the same size as the $+\mathrm{x}$ panel. It shares the block features as well as the geometry of the outer portions of the cutouts. Towards the center of the panel is a square system of struts with four holes at the corners. This cutout 
was implemented in the design to provide space for acrylic panels which house the electrical boards. Without these acrylic inserts, the electrical boards would have nonsquare hole patterns and would therefore require an asymmetrical strut geometry. Additionally, it was predicted that the electrical architect would evolve throughout the project and that some of the boards may need to be swapped or new ones added. It was therefore determined that an acrylic board insert would be much easier to update than having to machine a new aluminum panel.

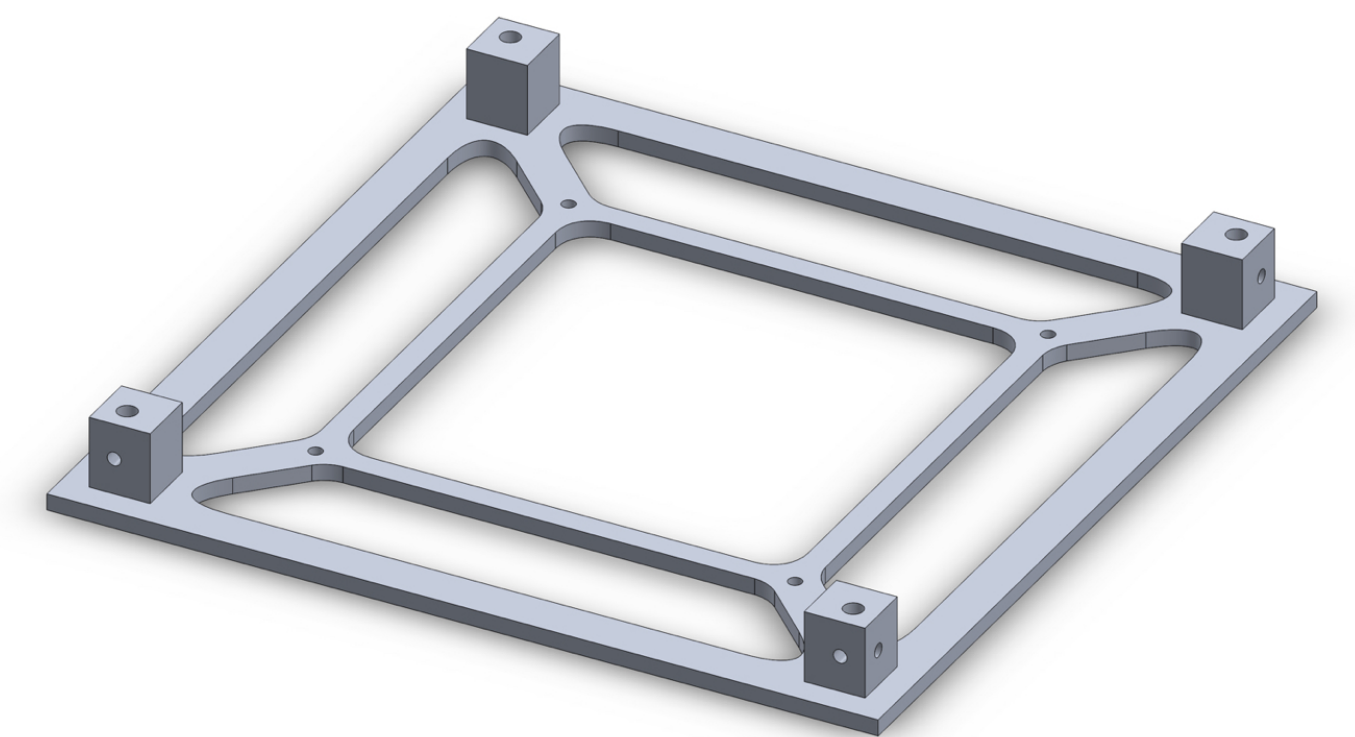

Figure 5.10: Final design of the $-\mathrm{x}$ side panel.

All features intended for the wheels, including the central hole and the towers, exist on two of the four minor panels. One of these panels is shown in Figure 5.11. These two side panels don't have the corner blocks. Instead, they are designed to mate to the master panel. The shallow extrusions are necessary features for the low profile hex screws to be flush to the outer surface of the panels while leaving enough material between the head of the screw and datum B.

The overall dimensions of these panels are $14.4 \mathrm{~cm}$ in width and $14.67 \mathrm{~cm}$ in length. 
This is because they are sandwiched between two $3 \mathrm{~mm}$ thick master panels, which cause the width to be $0.6 \mathrm{~cm}$ smaller than the $15 \mathrm{~cm}$ of overall width of the cube, and neighboring one $3 \mathrm{~mm}$ thick side panel with a nominal gap of $0.3 \mathrm{~mm}$. In order for the $\mathrm{x}$-shaped struts to be centered when the cube is assembled, they had to have an asymmetric geometry.

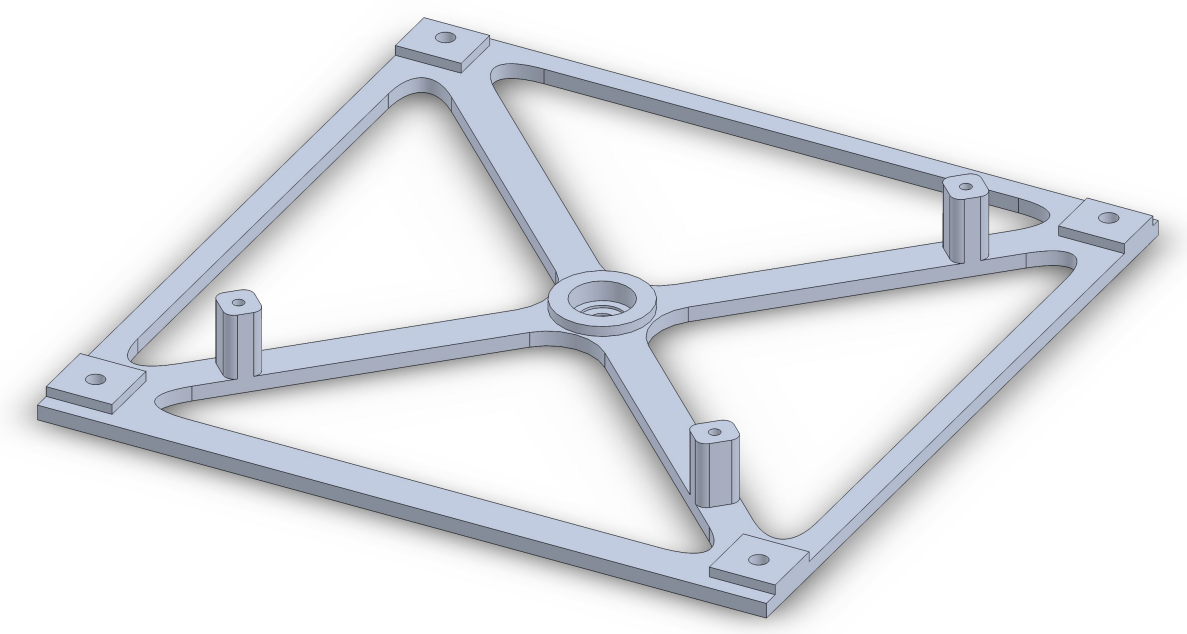

Figure 5.11: Final design of the $+\mathrm{z}$ side panel.

The other two minor panels share features with the $-\mathrm{x}$ panel and the two wheel bearing minor panels. They have the low profile extrusions on the four corners like the other minor panels and the square strut geometry and large central cutout designed for the electrical boards like the -x panel. One of these is shown in Figure 5.12 . 


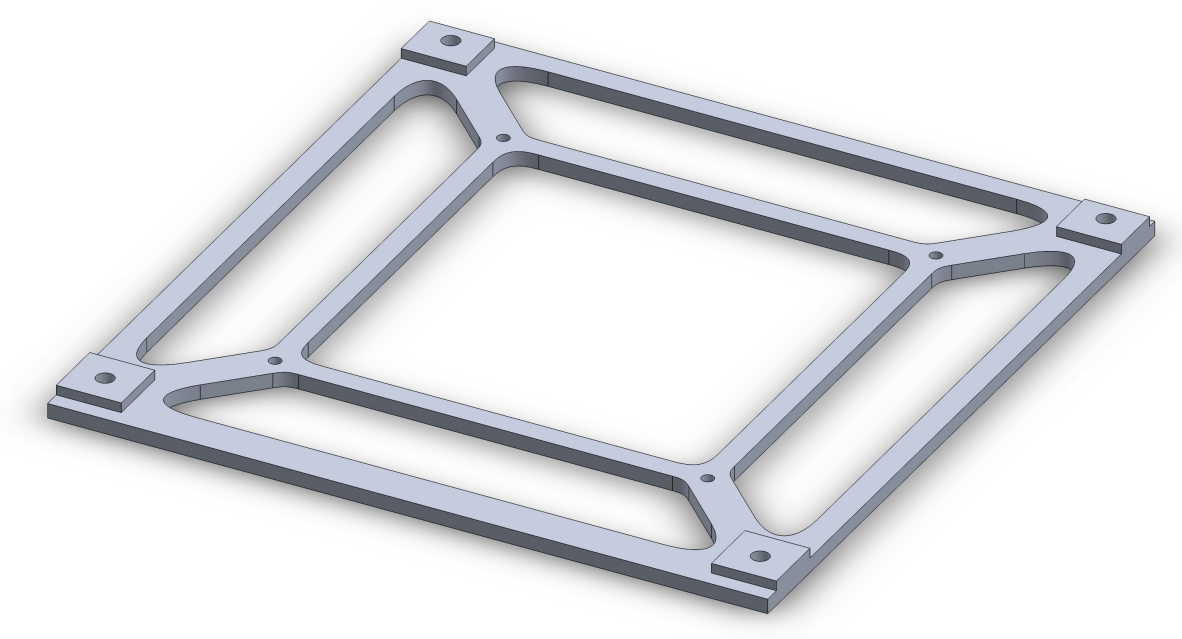

Figure 5.12: Final design of the $-\mathrm{z} /-\mathrm{y}$ side panels.

\subsubsection{Acrylic Panels}

At the beginning of the project, it was unclear how many and which electrical boards would be required for the system. Therefore, it was decided to design the upper panels of the cube with adaptability in mind. Another major driving force behind the final design of the acrylic panels was, once again, symmetry. The boards could have gotten screwed directly to the structure but in order for the screws to contact material the struts of the panels would have needed to be of odd geometric shapes.

For the reasons outline above, it was determined that a good design consisted of acrylic panels which would create an interface between the structural panels and the electrical boards. Any changes in electronics would then require new acrylic panels which could be cut with a laser and therefore very easy and cheap to make. Additionally, the aluminum structure could be symmetric, making the cube more aesthetically pleasing and simplifying the machining process.

The acrylic panels have four holes at the corners of an $86.5 \mathrm{~mm}$ square which are used to fasten them to the aluminum panels. Smaller clearance holes are scattered 
throughout the panels for placement of the electronics.

Figure 5.13 shows one of these acrylic panels. This one in particular had two electrical boards, the ESCON 36/6 and BNO055 modules on it for the first revision, subsequently one ADC board was added, and finally two more were installed into the acrylic panel. Modifying this panel for the boards was very easy and quick.

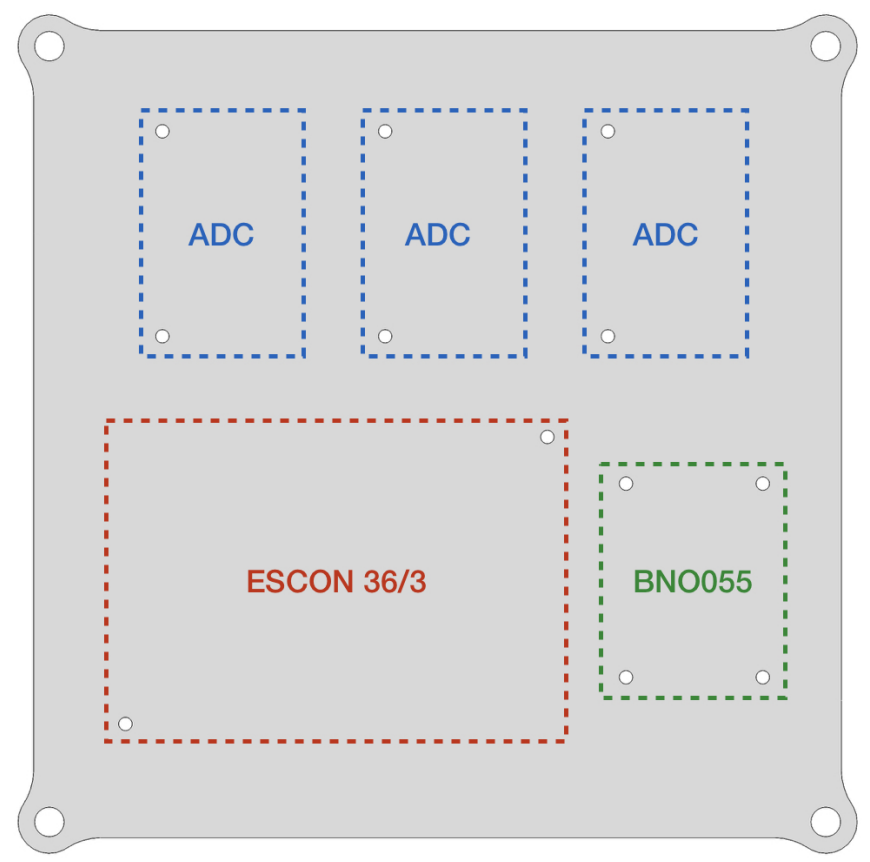

Figure 5.13: CAD model of the acrylic panel which the three ADCs, the IMU, and one of the motor drivers mounts to. 


\subsection{Motor Mounts}

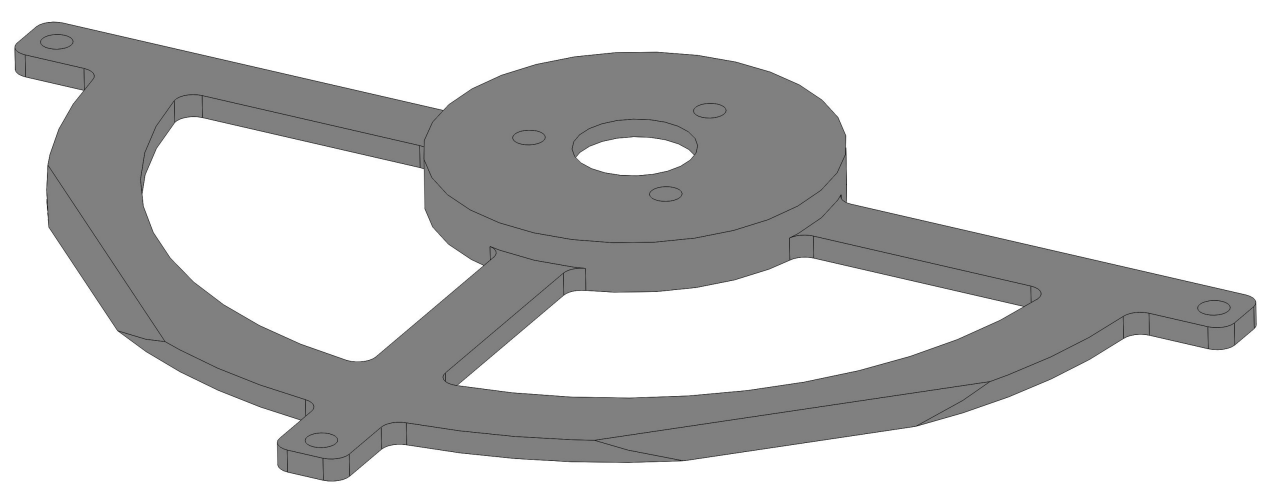

Figure 5.14: CAD model of a motor mount.

The motor mounts are structural aluminum components which act as a connection between the motors and the panels. The motors are attached to them with three screws. After the reaction wheels are assembled to the motor shafts, the motor mounts are mated to the appropriate wheel panels. Figure 5.1 shows the motor mounts in the cube in dark gray.

The cylindrical feature at the center of the motor mount matches the radius of the motor. When the two components are assembled, this cylinder is coradial and concentric to the motor's housing.

One aspect that was decided early in the design process was the number of spokes the motor mounts should have. Having only a single spoke was thought to potentially cause issues with cantilevering of the beam and was therefore eliminated as an option. Two opposite spokes seemed like a good idea, since any bending of the component would result in the motor translating away from or towards the side panels rather than rotating. However, a design with three spokes was chosen in the end due to it allowing for the half-circle shaped bars which could provide surfaces for batteries to 
rest up against in the case this became desirable. Additionally, they help to keep cables out of the spinning wheels. A fourth spoke was not added to keep the weight and center of gravity of the cube low.

The outer surfaces of the half-circle bars were designed to be coradial with the outer surfaces of the wheels. This caused neighboring motor mounts to intersect. To mitigate this problem, material was removed from each of the motor mounts, hence the 45 degree cut on the sides.

\subsection{Wheel Stack Design}

The wheel stacks consist of several components - an electric motor, a motor mount, a mounting hub, a reaction wheel, and an external bearing. The motor has two bearings in it which fully constrain the shaft. The third bearing was added to the end of the shaft to support any imbalance of the reaction wheel. To keep the system from being over-constrained, the motor and motor mount assemble can move laterally relative to the panel before being screwed down. Additionally, a ball bearing was chosen for use at the end of the shaft to allow for some rotational freedom about the two axes perpendicular to the shaft of the motor.

In order to attach the wheels onto the shaft without backlash or movement, the wheels were screwed to the mounting hubs with four M3x6mm screws. These hubs have set screw which are tightened onto a flat spot on the shaft. Figure 7.12 shows the flat spot that was cut into the shafts for the set screws. 


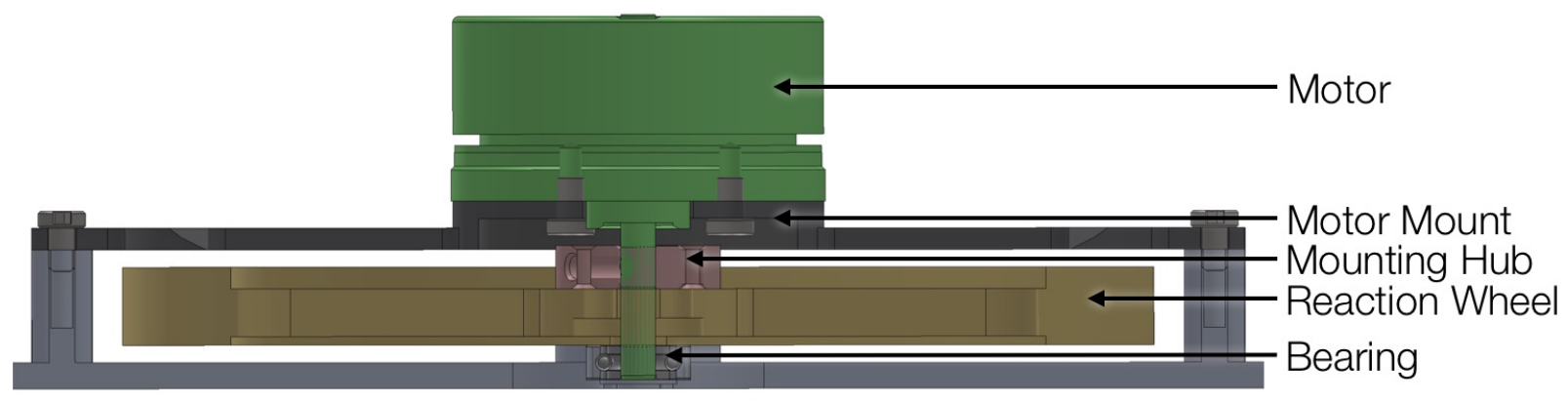

Figure 5.15: Wheel stack design, showing the motor, motor mount, mounting hub, reaction wheels, bearing, and side panel. 
Chapter 6

\section{ELECTRICAL DESIGN}

\subsection{Architecture}

The electrical system architecture is shown in Figure 6.1 below.
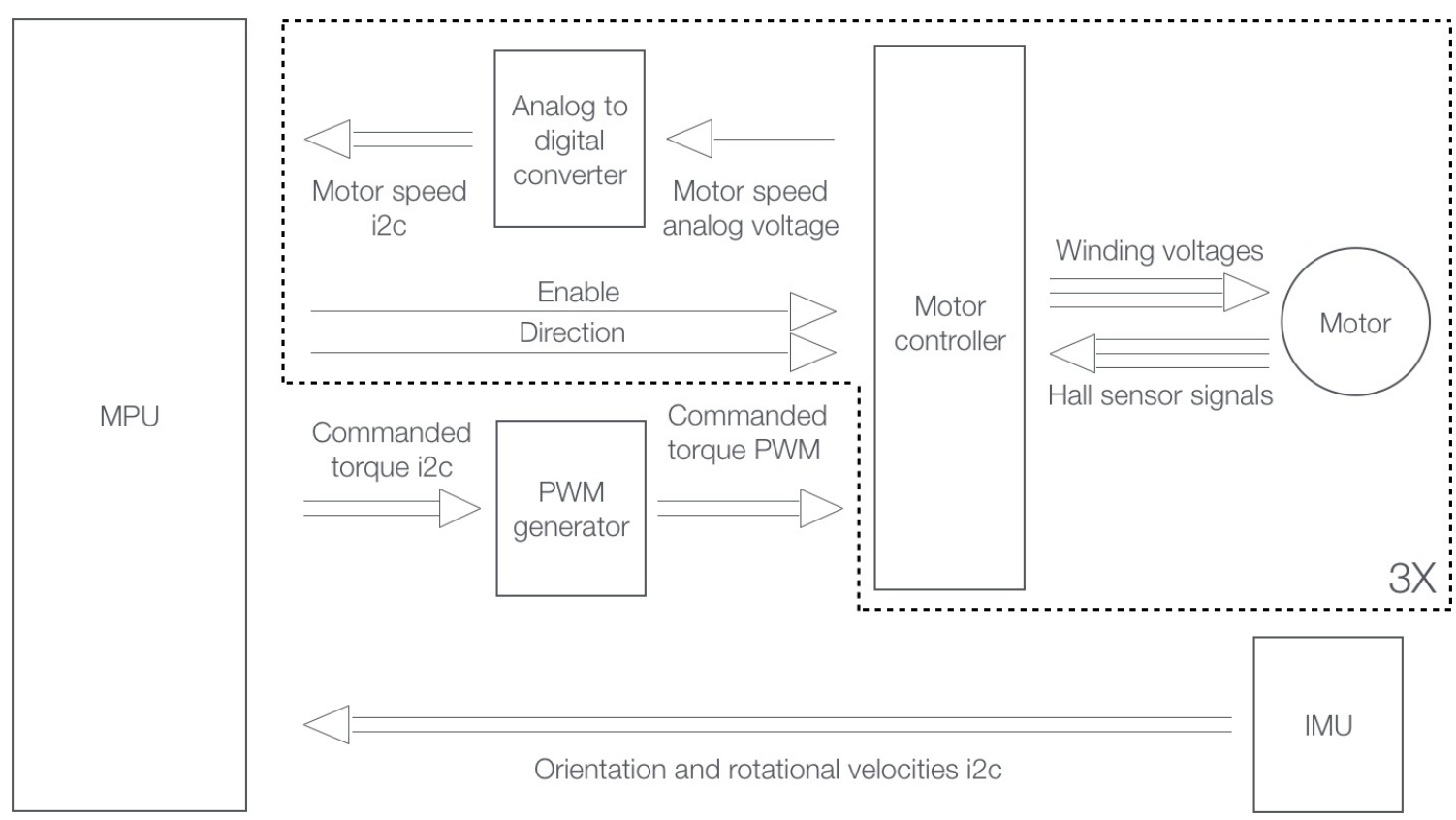

Figure 6.1: The overall electrical system architecture of the balancing cube. Note that the system contains three motors and motor drivers. Only one set is depicted.

The cube senses its orientation and rotational velocities by ways of an Inertial Measurement Unit, or IMU. Meanwhile, motor drivers sense the velocity and direction of rotation of the reaction wheels using hall sensors. They then relay this information to the on-board computer via analog to digital converters. The computer then processes the control algorithm mathematics and commands the motor controllers to apply a certain amount of current to the motors via a PWM generator board. 


\subsection{Computer}

The Raspberry Pi 3 Model B+ was selected as the main processor of the cube. It was primarily chosen for its ability to run Simulink. Its high clock rate was also a factor in the decision to use it. Since it was not well understood in the beginning of this project what kind of performance was required, a processor with much more capability than what was actually required was welcomed.

\subsection{Motors}

DC brushless motors spin the three reaction wheels. Maxon-made EC45 flat motors capable of outputting 50 Watts were chosen for their energy density and small size. They contain eight pole pairs to control the rotor and three hall sensors for measuring of the rotational position. Their stall torque is $780 \mathrm{mNm}$ which was predicted to be more than enough. They also spin up to $6700 \mathrm{rpm}$, which again was thought to be plenty. The decision of using these motors was made relatively early on before it was known how much torque or speed would be required. They are therefore quite a bit more powerful than necessary.

\subsection{Motor Drivers}

Escon 36/3 motor drivers were chosen to drive the DC brushless motors for several reasons. First, the motors and motor drivers have plug-and-play functionality. Second, they post-process the hall sensor data and populate a register with the speed of the motors which was expected to be much easier than decrypting the hall sensor signals. Third, the motor drivers have a closed loop current control method which, using a simple scalar gain, can be turned into closed loop torque control. Again, this feature made controlling the motors very easy. 


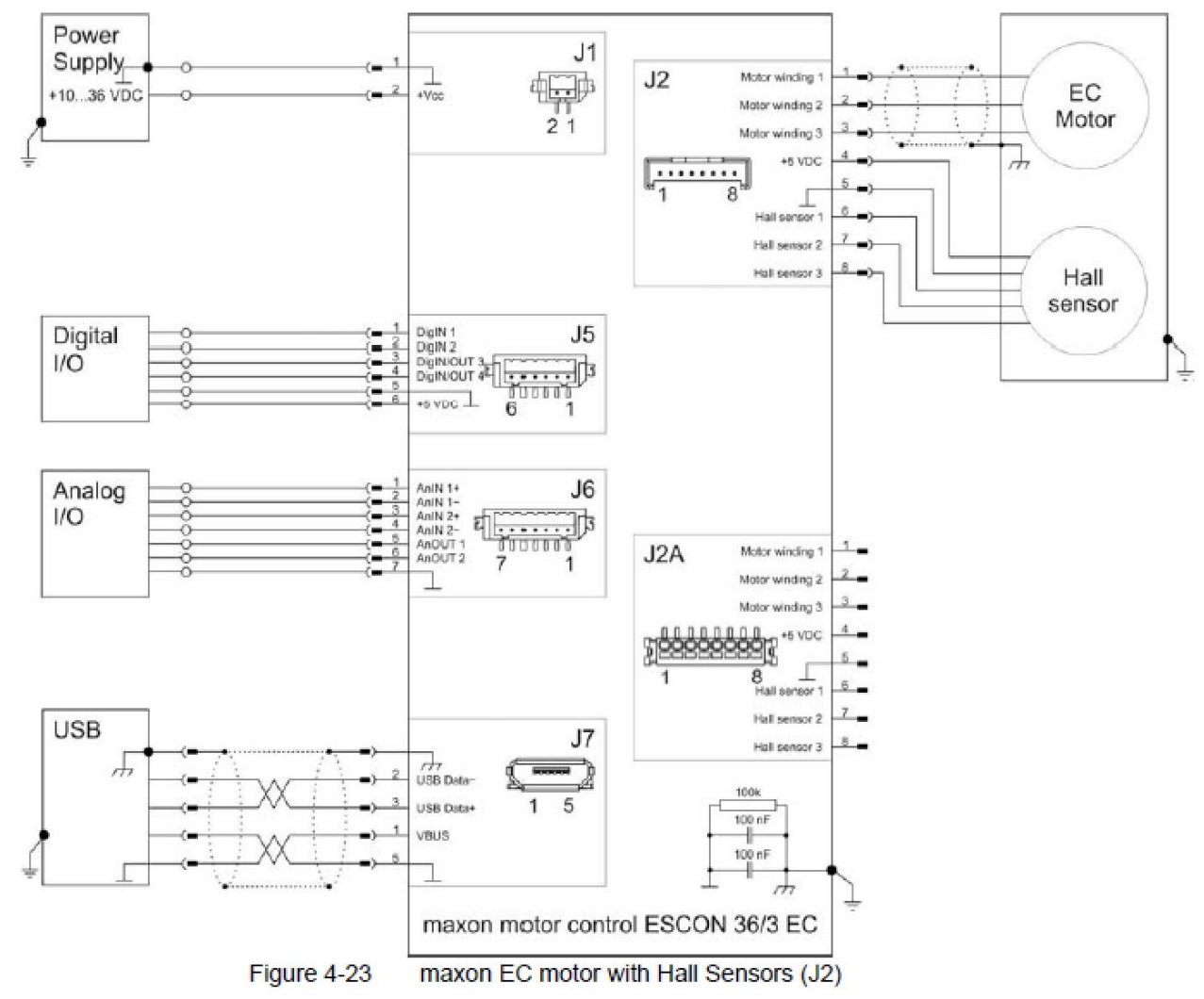

Figure 6.2: Pinout diagram of the ESCON 36/3 motor drivers.

As shown in the top right corner of Figure 6.2, the motors had to be connected to the J2 connector with 8 cables. Power was supplied to the drivers by an external power supply to the J1 connector. Digital I/Os were used to set the direction (binary) and desired current of the reaction wheels (PWM from the PWM generator board). One of the analog I/O pins outputted the velocity of the wheels. This signal was fed to one of the ADC boards, converted to a digital signal, and sent on to the Rasperry Pi.

\subsection{IMU}

The IMU board consists of a 9-DOF Bosch-made BNO055 chip mounted on an Adafruit breakout board which not only measures acceleration, rotational speeds, 
and magnetic fields, but also post processes the results. The BNO055 contains filtering mechanisms to return cleaner data and outputs its orientation (as a quaternion or in Euler angles), the gravity vector and the linear acceleration of the chip.

The device was chosen for its capability and easy of use. Since the sensor outputs its orientation, the orientation did not have to be calculated from raw acceleration and gyrometer readings. It posts measurements to its registers at a rate of up to 100 $\mathrm{Hz}$ and communicates via $\mathrm{I}^{2} \mathrm{C}$.

\subsection{ADC Board}

Since the motor drivers output an analog signal and the Raspberry Pi does not have any on-board ADCs, separate ADC boards were installed into the cube. Adafruitmade ADS1015s were chosen for this application. For reasons outlined in Figure 8.3.2, three ADC boards were used. Each of them is capable of measuring four different signals, but only one port was used from each of them. These chips populate a register which was read via $\mathrm{I}^{2} \mathrm{C}$ by the Raspberry Pi.

\subsection{PWM Board}

The Raspberry Pi processor does not have enough hardware PWM generation capability. With the two PWM hardware channels, the three motors could not be controlled. Therefore a hat was added to the Raspberry Pi which generates up to 16 channels of PWM and is controlled by $\mathrm{I}^{2} \mathrm{C}$. The main chip on this device is the PCA9685 made by NXP Semiconductors. 


\section{$6.8 \quad \mathrm{I}^{2} \mathrm{C}$ Bus}

Many of the chips in the system communicated with the $\mathrm{I}^{2} \mathrm{C}$ interface. The allocation of the addresses is shown below.

$$
\begin{aligned}
& \text { 0x28 }=\text { BNO055 } \\
& 0 \times 40=\text { PCA9685 } \\
& 0 \times 4 A=\operatorname{ADS} 1015-\text { motor } 1 \\
& 0 \times 48=\operatorname{ADS} 1015-\text { motor } 2 \\
& 0 \times 4 B=\operatorname{ADS} 1015-\text { motor } 3
\end{aligned}
$$


Chapter 7

MANUFACTURING AND ASSEMBLY

\subsection{Overview}

Creating the cube involved a combination of CNC machining, laser cutting and making cables. Everything, including the planning of the CNC toolpaths, CNC operation, fixture design and cable making was done in-house.

\subsection{CNC Machining}

The cube's design calls for 12 aluminum parts - six side panels, three reaction wheels, and three motor mounts. These components were manufacturing using a HAAS VF3SS 3-axis mill.

Starting with the CAD model, the paths, feeds, and speeds of the cutting tools were specified with the CAM (Computer Aided Manufacturing) software HSMWorks. The $\mathrm{G}$ code that it created was transferred to the mill. An aluminum block was mounted into a vise, probed for purposes of creating a datum structure, and cut with various cutting tools. Specialty fixture had to be created to hold on to the parts during some of the cutting processes. Section 7.2.5 describes the design of the fixtures used.

\subsubsection{Toolpath Planning and G-code Generation}

G code consists of sequential commands which are executed by a CNC machine to move the tool relative to the work piece. For the creation of the parts for the cube, the CAM software HSMWorks was used to generate this code. The software incorporates 
into SolidWorks in the form of an add-in. It then provides a graphical user interface to design tool paths.

\subsubsection{Manufacturing Reaction Wheels}

The milling process which resulted in the reaction wheels consisted of several stages. First, with the aluminum block in the vise, a facing operation removed the top layer. Second, the profile of the reaction wheel was cut from the block. Figure 7.1 shows the work piece after those two operations.

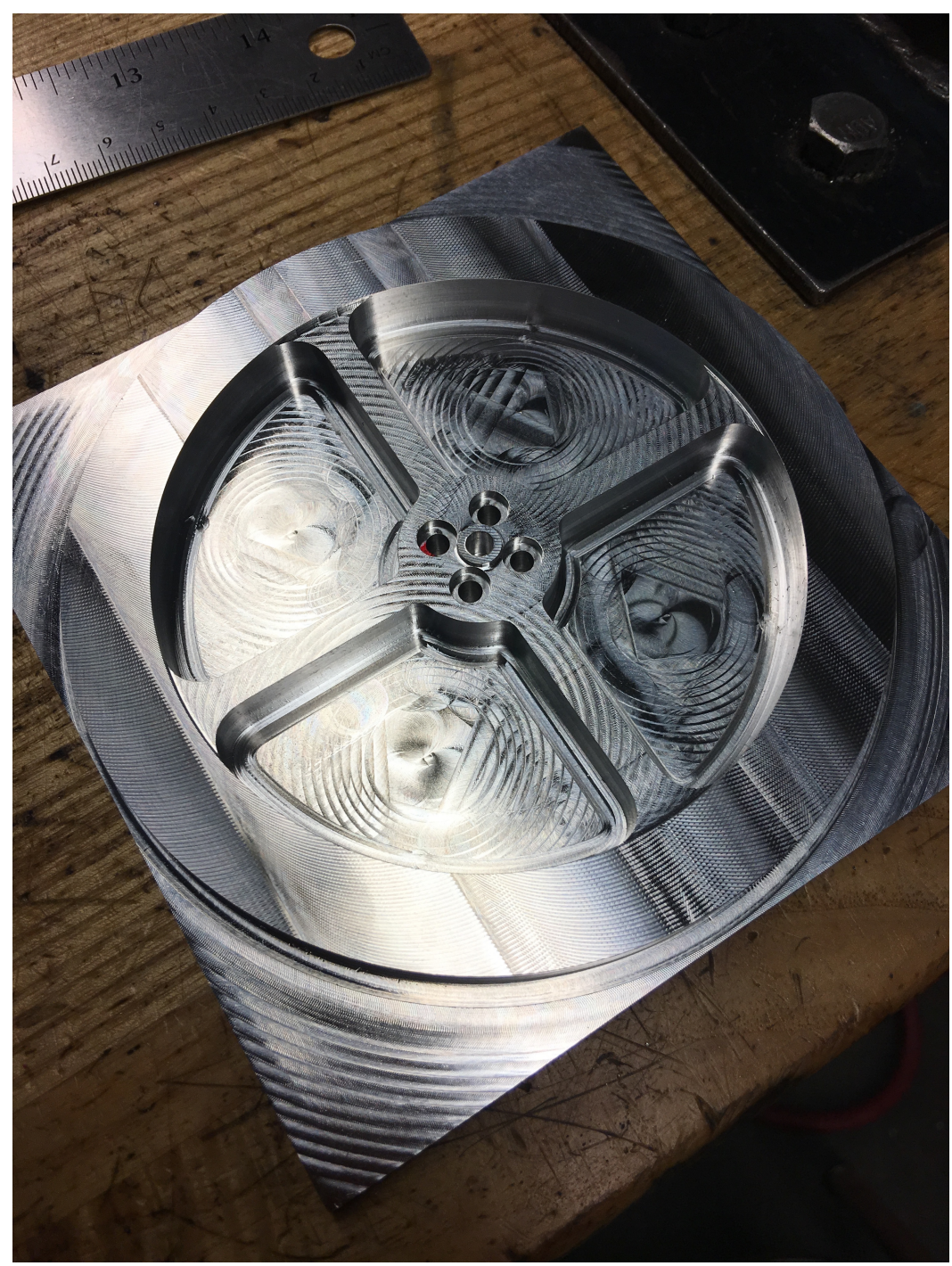

Figure 7.1: Reaction wheel work piece after first machining job. 
All features of the wheels requiring high positional accuracy relative to each other were cut within one stage. They were performed without removing the aluminum from the vise and without changing the datums. The most important tolerance was the concentricity of the reaction wheel relative to the central hole. Cutting these two features from the same side ensured that the reaction wheels would have very little rotary imbalance.

Next, the work piece was clamped into a special fixture which is further explained in Section 7.2.5. Then, the remaining aluminum was removed from the work piece.

At the end of each job, many of the edges were chamfered to get rid of burrs.

The most difficult part of creating the reaction wheels was the removal of material to form the central hole. The hole diameter had the most tightly specified tolerance of any parts of the cube. It took many attempts to successfully create usable reaction wheels. The problems and lessons associated with manufacturing these holes are listed in Section 7.2.6. 


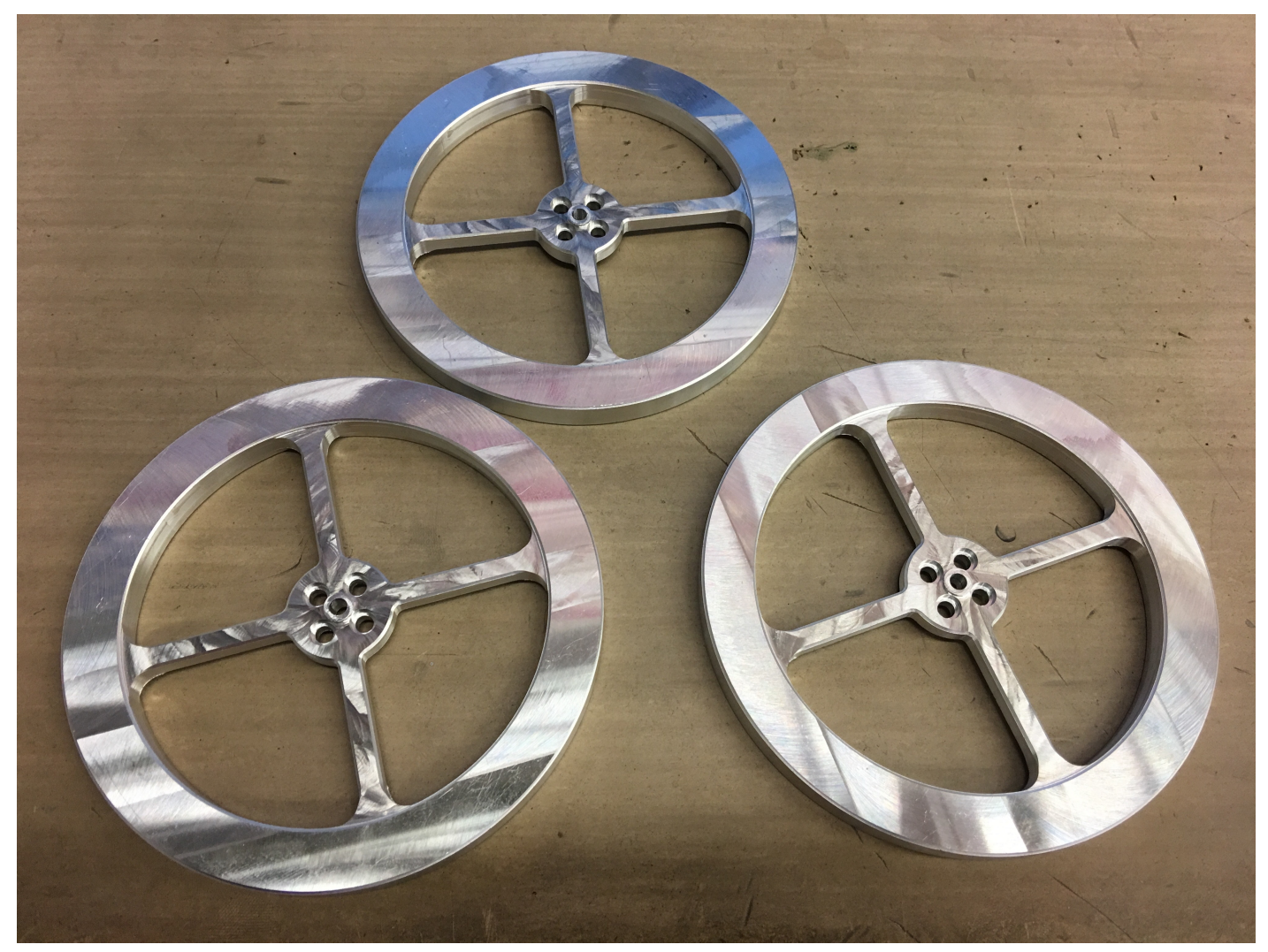

Figure 7.2: Finished reaction wheels.

\subsubsection{Manufacturing Side Panels and Motor Mounts}

Of the six side panels, five were unique. As explained in Section 5.2.1, the master panels have threaded holes for the other side panels to screw into. These two parts were the most complex to machine as they required material removal from all six sides.

The machining process of all side panels started with squaring aluminum blocks. Each of the two master panels was then placed on a side, gripped by a vise, and two holes were cut and then threaded into the top surface after it was faced. Forming taps were used to create the threads. Then, the work piece was flipped 90 degrees and the next two holes were cut and threaded. This was done two more times until all four sides had holes cut into them. For the datum structure, the bottom surface of the 
vise was set as the $\mathrm{x}-\mathrm{y}$ datum for all operations. This resulted in the square pieces having the correct widths and lengths. The front surfaces were set as the x-z plane, guaranteeing that all holes would be the same distance away from the outer surface of the cube. Finally, the same two surfaces of the squares were used as the y-z datums for the hole cutting operations. Of the two surfaces, the one which was parallel to the axis of the respective hole became the datum for that specific operation.

The following procedure applied to all six panels. The aluminum blocks were laid flat and strapped into the vise, with the soon-to-be outer surfaces of the cube being accessible by the cutting tools. Material was removed from the work pieces until the desired features came into being. These included four counter-bored through-holes and chamfers around the edges of the panels and a central hole for the wheel panels. The pieces were then flipped and attached to a fixture, which is described in detail in Section 7.2.5. The remaining material was then removed. A few specific machining operations are explained below.

Each of the three wheel panels has three towers which the motor mounts screw into. These features defined the thickness of the aluminum stock used. Most of the remaining features of the panels did not protrude as far, so lots of material had to be removed from these parts. In order to do this quickly and without causing high changes in stresses on the tool, an adaptive clearing operation was used. This type of cutting operation consists of a tool path which is optimized for consistent forces on the cutter throughout the motion. For this specific operation, a 0.375 " diameter carbide cutter removed $0.07 "$ throughout each pass. This cutter was moved quite quickly at $250 \mathrm{in} / \mathrm{min}$ with a cutting depth of approximately half an inch. Figure 7.3 shows the work piece following this operation. The lines in the material show the path which the tool followed. 


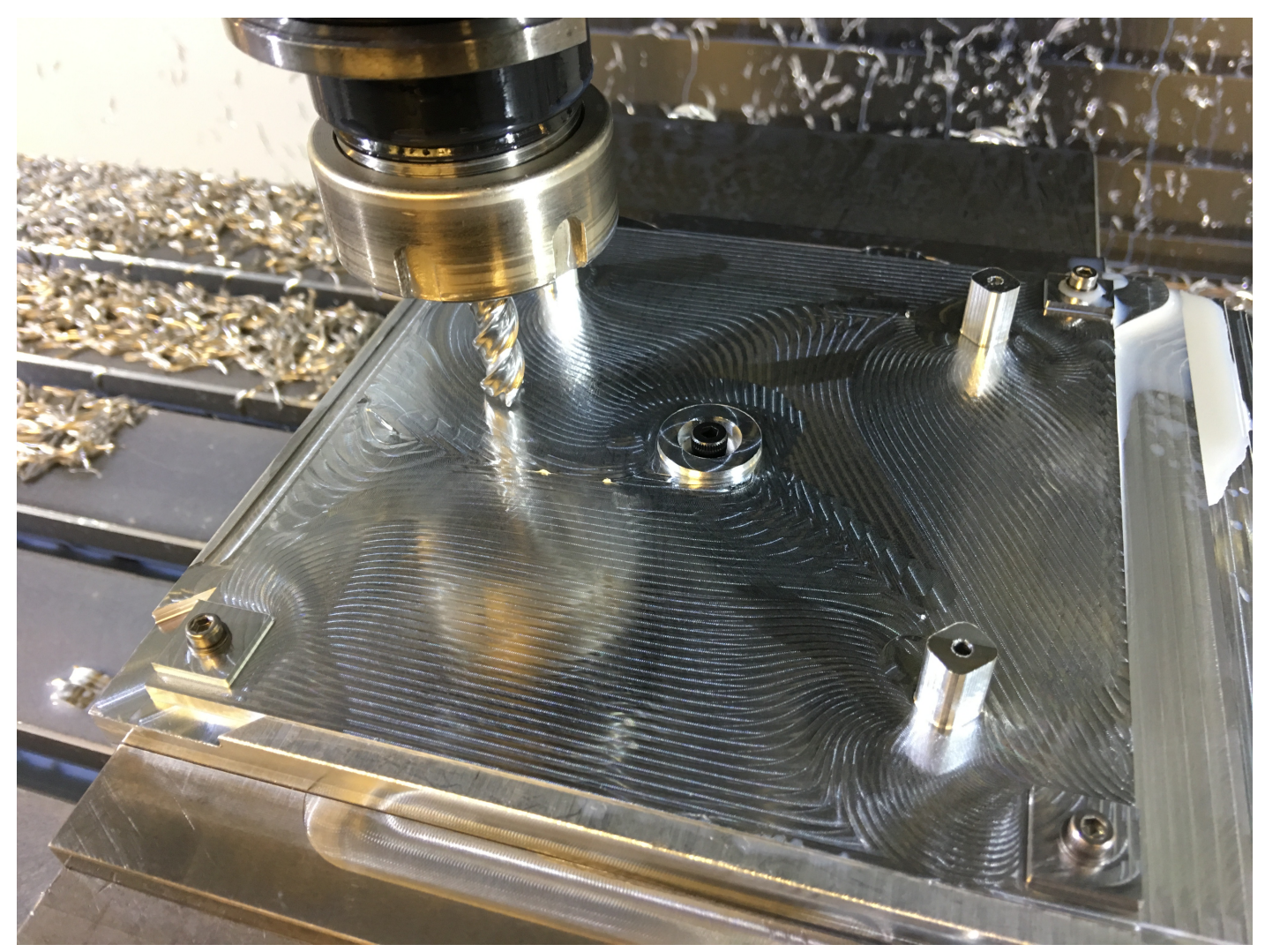

Figure 7.3: A wheel panel after the adaptive clearing operation. The lines in the aluminum show the path of the tool.

The other operation of interest was the cutting of the features for the bearings. The design of the cube called for the bearings to be press-fitted into the wheel panels. Therefore, the cylindrical cutout had to be quite accurate. A roughing end mill first took out most of the material except for about 0.015 " from the bottom of the cut and from the sides. Then, a smaller cylindrical section was cut out from the floor of the bearing surface to allow for a gap between the inner race of the bearing and the panel. Next, a finer and more precise cutter was used to repeat the first cutting operation, effectively shaving off the 0.015 " of material that was left on the work piece. This pass was then repeated to ensure that any flexing of the tool would not affect the accuracy of the hole.

A finished wheel panel is shown in Figure 7.4 . 


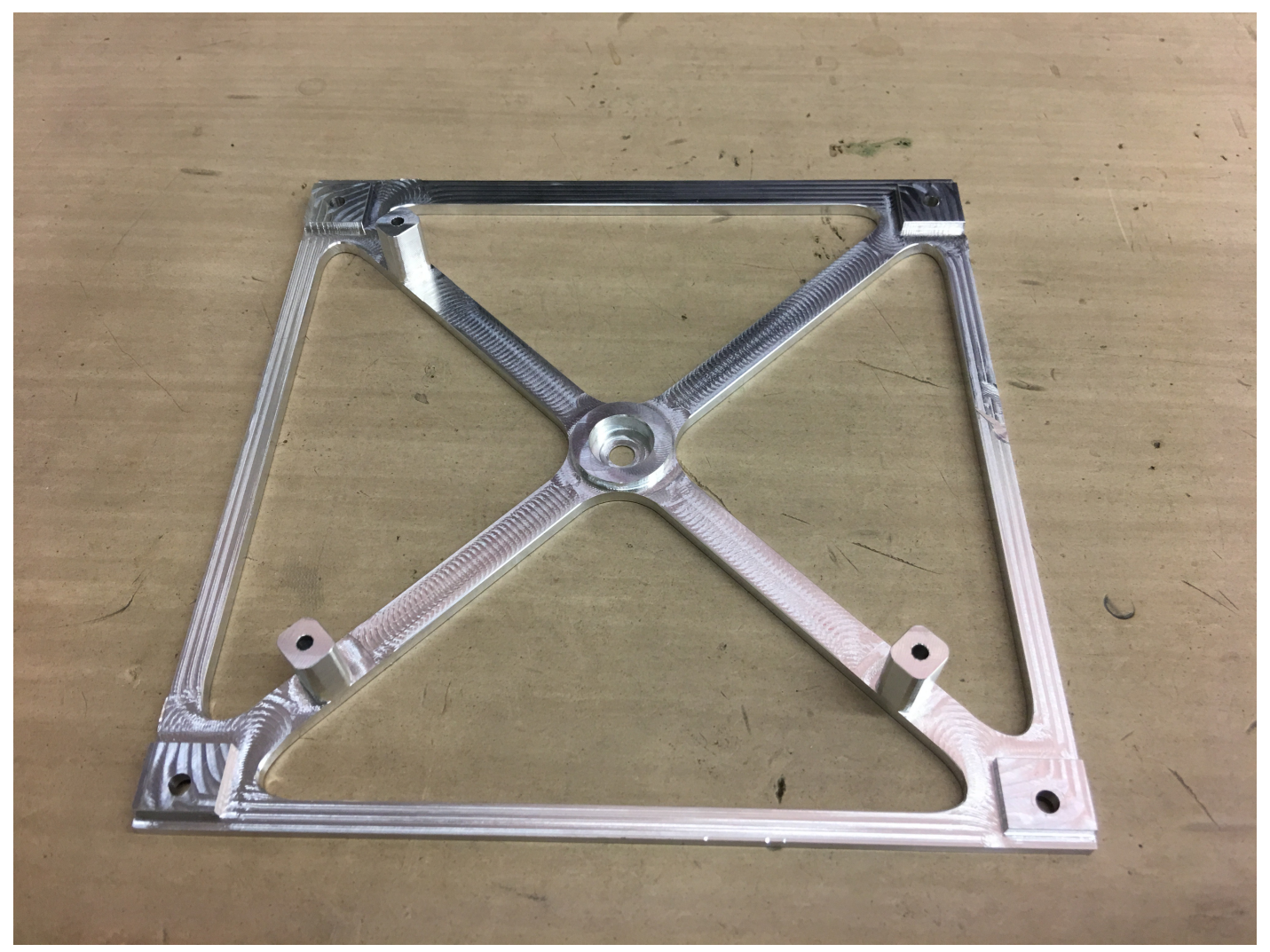

Figure 7.4: Finished wheel panel.

\subsubsection{Motor Mounts}

Machining the motor mounts proved to be much easier than the reaction wheels and side panels. These components did not have features requiring close tolerances. Each of the holes had a $\pm 0.3 \mathrm{~mm}$ tolerance which is well within machining capability. The work pieces were machined from two sides. The bottom was cut first. Then, similarly to the side panels, the parts were flipped upside down and screwed onto the fixture described in Section 7.2 .5 .

\subsubsection{Fixturing}

Three fixtures were used for the creation of the parts of the cube. The first one supported the reaction wheel, the second the side panels, and the third the motor 
mounts.

In order to hold the reaction wheels, the first fixture had to have a cylindrical cutout which could contact the outer surface of the reaction wheels and press inwards firmly. To be able to apply a normal force to the wheels, the fixture was made from two halves with a gap in between. Each of the halves was fixed to one side of the vise and as the vise closed, the halves pressed against the outer surface of the wheel.

The fixture had three datums. One was the surface upon which the wheel rested. To enable the spindle probe, a device which measures the location of objects in a mill, to reach this datum when a wheel is in the fixture another cut was made into the fixture. The bottom of this cut was co-planar to the intended datum surface. Since both of these surfaces were cut in the same operation, it was assumed that the only positional and angular tolerances were due to the machine tolerance which would not cause any issues. The second datum was chosen to be the front surface of the front half of the fixture. Lastly, the third datum was chosen to be the right surface of the same block. Lessons from designing and cutting this fixture are described in Section 7.2.6. 


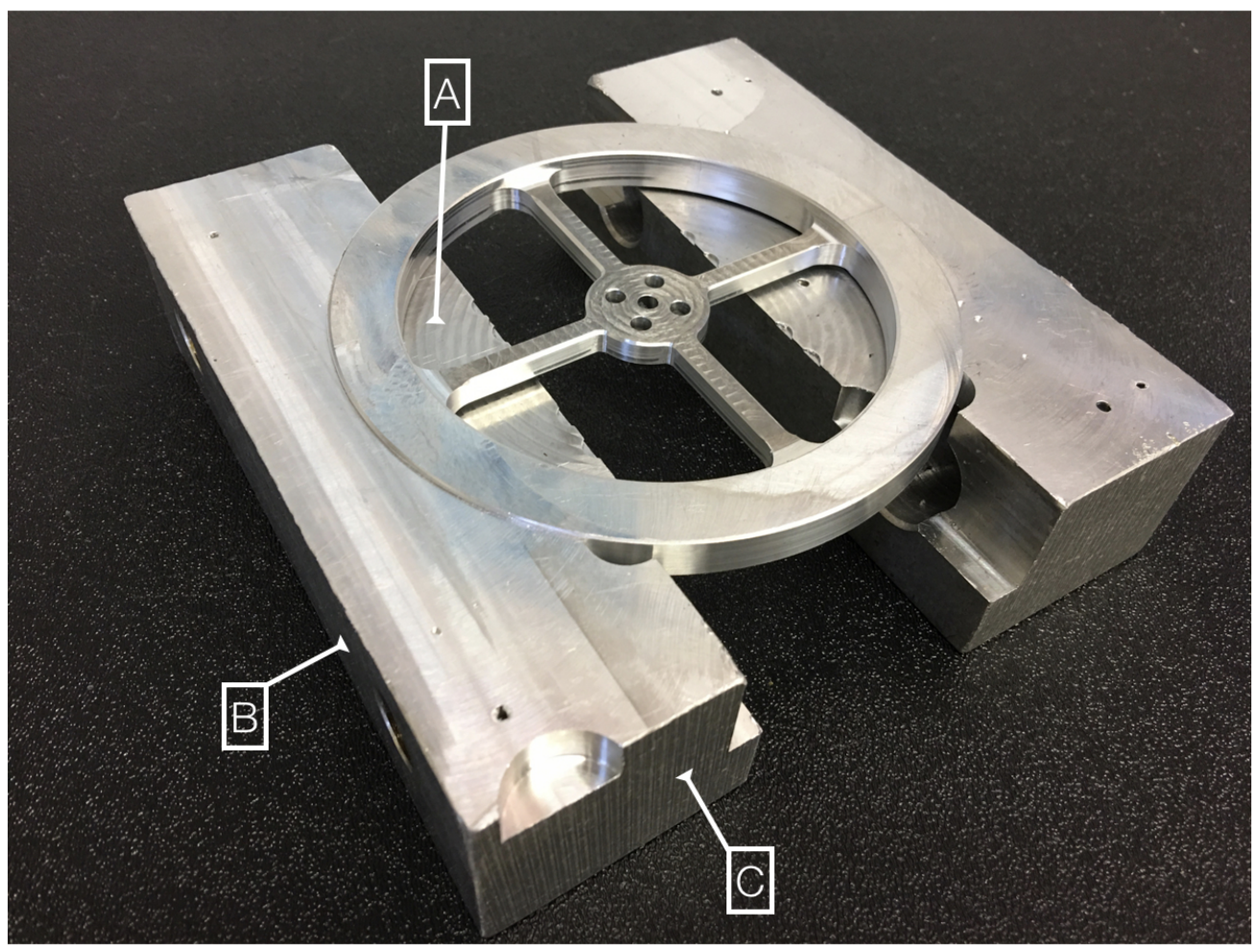

Figure 7.5: Picture of reaction wheel fixture showing the datums.

The second fixture was designed as a flat plat with two perpendicular walls. The inner surfaces of these walls became the datums restricting the wheel panels in the $\mathrm{x}$ and $y$ direction. The flat surface of the plate became the datum for the $\mathrm{z}$ direction. Five holes were drilled into the plate - four holes in a square shape allowed for all panels to be screwed down, the fifth added additional support for the wheel panels and was colinear to the axis of the bearing features.

This fixture was first used for the minor panels. Following the processing of the four minor panels, material was removed from the datum A wall. The modified datum $\mathrm{A}$ and datum B were then used for the master panels. Figure 7.6 below depicts this modification. The trench feature adjacent to the datum $\mathrm{B}$ of the fixture, shown in figure 7.7, shows that two cuts were made. The first was to form datum $\mathrm{B}$ for the minor panels, the second for datum B used for the manufacturing of the master panels. 
During the milling process, several screws holding down the side panels had to be removed temporarily. One of the first operations was to machine the side panel down to the appropriate height at the location of the screws. The appropriate screw was pulled so that the tool could move into the region and then put back when the cut was complete. Each of the screws were pulled sequentially so that at any given time all but one screw would hold the work piece to the fixture.

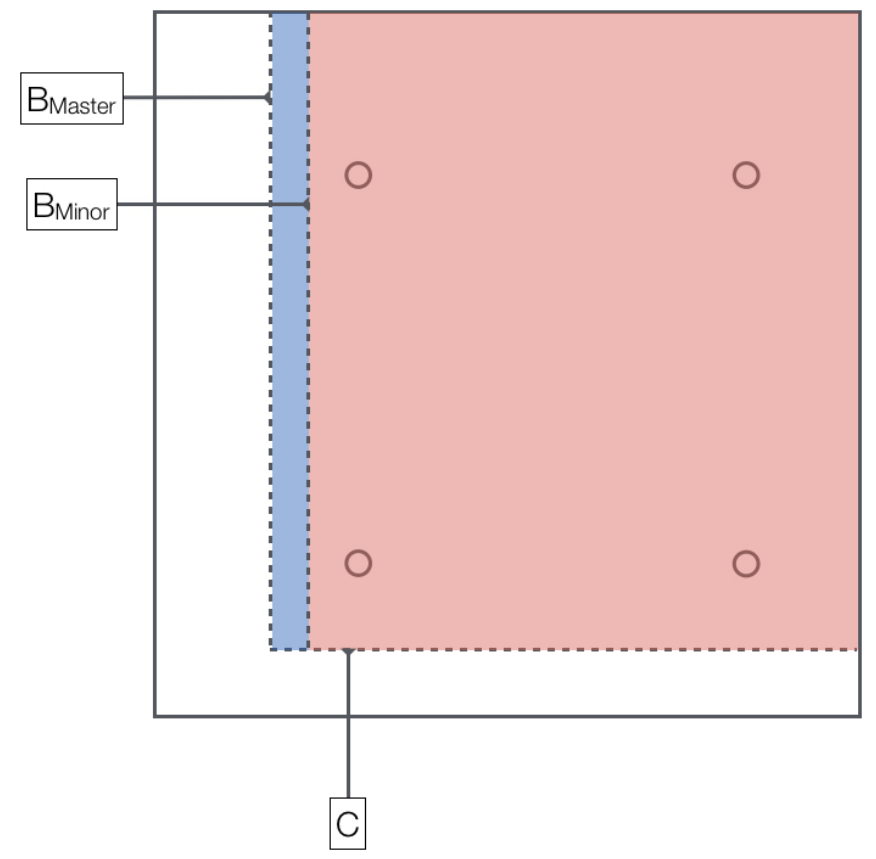

Figure 7.6: Illustration of the modification of the panel fixture. The minor panels occupied the red area. The fixture was then cut larger to accommodate the major panels which occupied both the red and blue areas. 


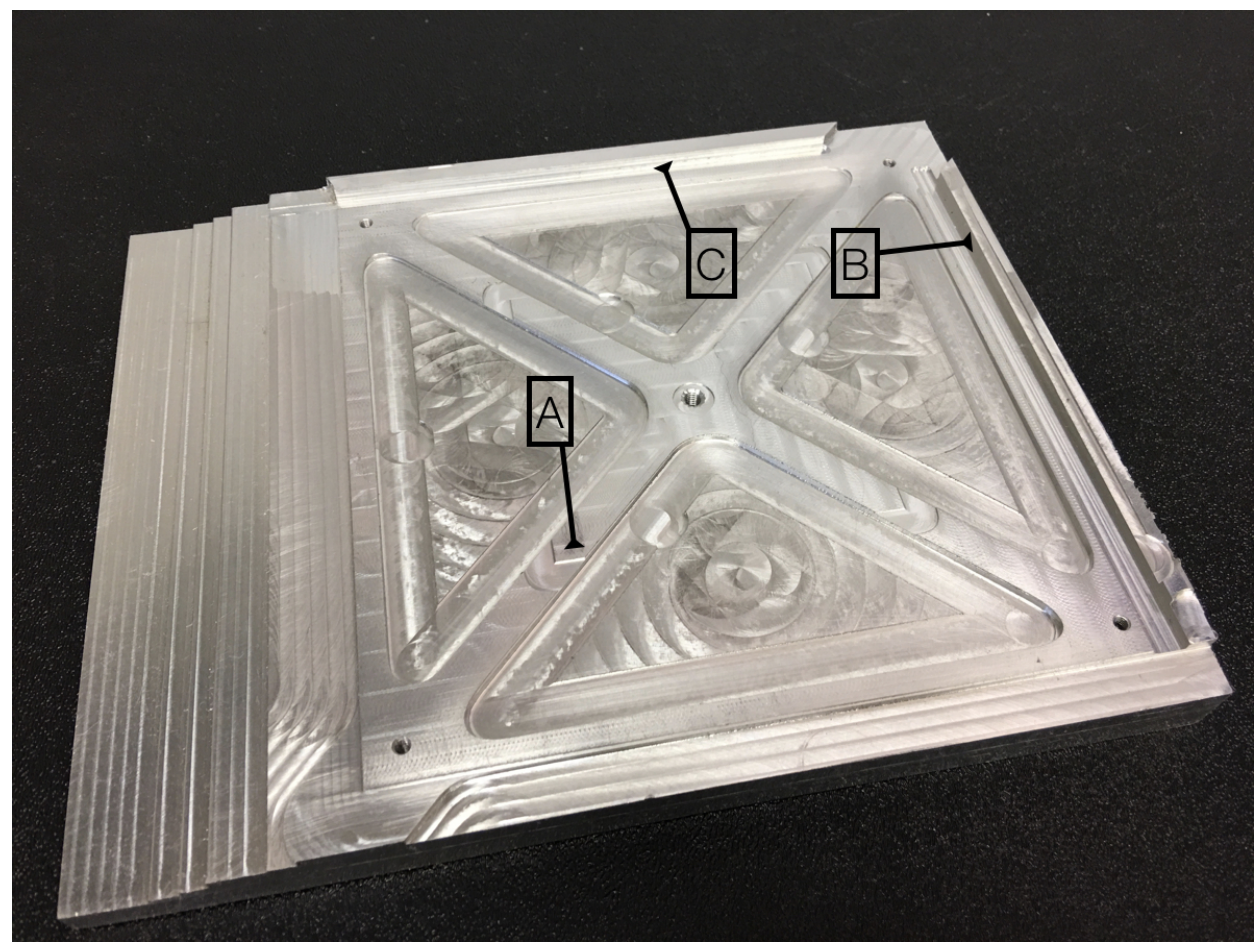

Figure 7.7: Side panel fixture with datums.

The third fixture was used to hold on to the motor mounts. It was designed as a flat plate with two perpendicular walls. The motor mount work pieces were flipped upside down after the first operation, placed onto the fixture, and slid to contact datums $\mathrm{B}$ and $\mathrm{C}$ shown in Figure 7.8 .

Figure 7.8 shows 14 holes. The six holes within the contour of the motor mount were used to hold the motor mounts to the fixture. Four of the surrounding eight holes (One of the two in each corner) were used to fix the work piece to the fixture. At first, only the outer four screws were installed. The features of the motor mount were machined before the six screws were installed to hold it down. Then, a small cutter was used to separate the motor mount from the rest of the work piece. After this operation, the motor mount was complete and held down by six screws. 


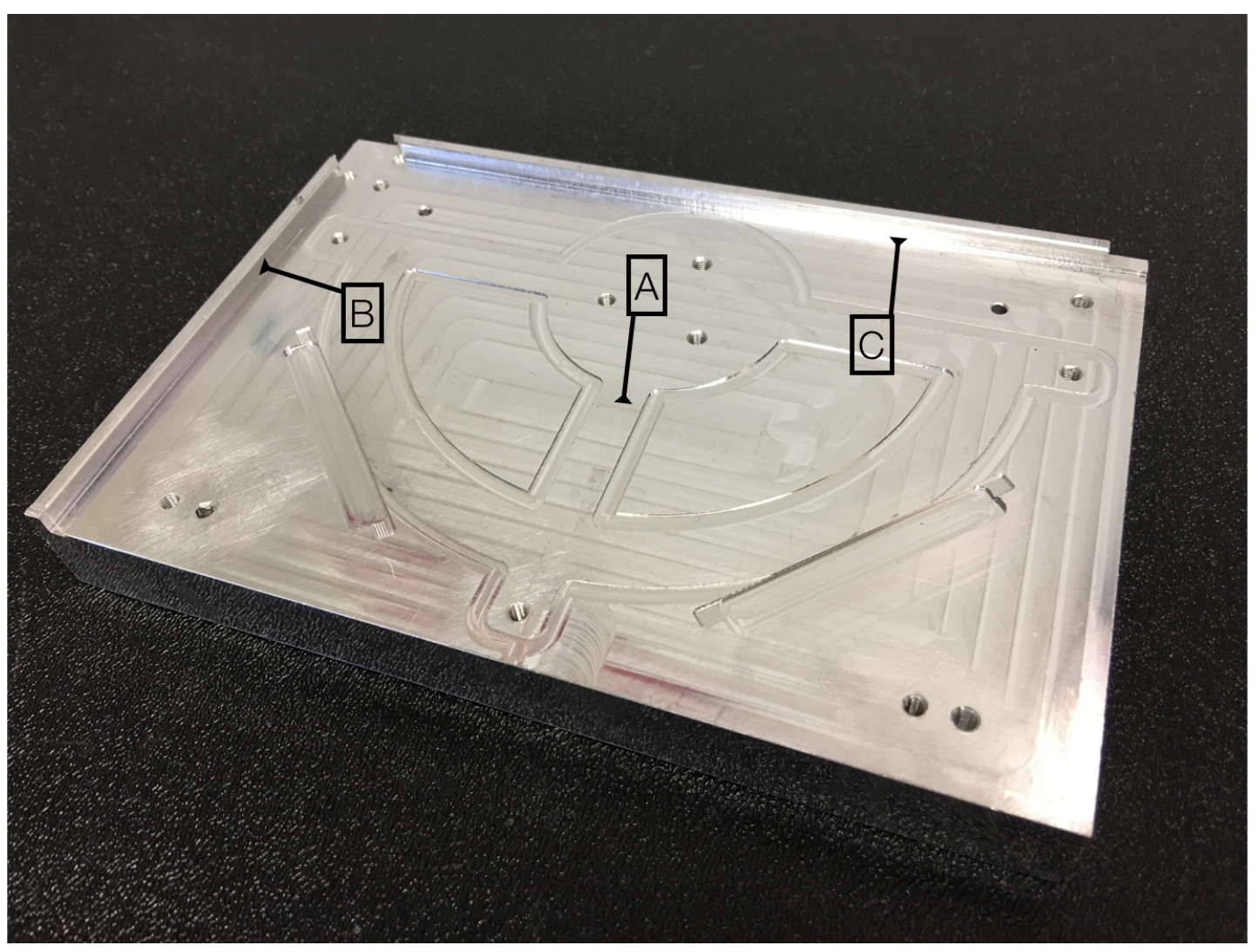

Figure 7.8: Motor mount fixture with datums.

\subsubsection{Lessons and Problems}

Many of the machining operations and processes did not go as expected. For several parts, multiple attempts had to be made until they came out satisfactory. The significant problems that occurred along with the things learned from them are described in this section. First, the details of cutting usable reaction wheel holes for a press fit onto the motor shafts is outlined. The lessons from chamfering edges follows that. Another issue concerned the radii of inner corners of fixtures. Lastly, issues associated with the reaction wheel fixture's datum structure are described.

Initially, a single reaction wheel was cut to determine the viability of creating these parts. A $4 \mathrm{~mm}$ diameter drill was used to cut the central hole with a nominal diameter of $4 \mathrm{~mm}$. After completion of the machining, the size of the hole was tested 
by pushing the reaction wheel onto a motor shaft. The hole was evidently too big. The problem with using a drill was the wobble of the drill. When the drill rotated, the slight angle of the drill relative to the chuck caused a larger-than-intended hole. Therefore, when the reaction wheel was placed on the motor shaft, the wheel had too much play and wobbled significantly.

The second attempt to make wheels was different than the first in that the hole was cut with an end mill rather than a drill. The end mill was programmed to descend into the material in a helical fashion, cutting material while it spiraled. The diameter of this cut was purposefully undersized. The operation was then repeated several times with slightly larger diameter helical tool paths until the motor was close to fitting in the hole. The reaction wheels' holes were intentionally cut to interfere with the shafts of the motors to make sure that they would mate tightly. After assembling the full wheel stacks, incorporating them into the cube, and spinning the wheels, it was found that the wheels wobbled. They did so with varying magnitudes, with the worst wheel wobbling up to $2 \mathrm{~mm}$. This was deemed unsatisfactory because it was predicted that spinning, wobbling wheels would induce vibrations and possibly issues with the control logic. The cause for this issue was likely the procedure of press-fitting the reaction wheels onto the motor shafts. This process was performed with an arbor press. The press did not have a way of datuming the motor shaft to the reaction wheel and therefore the parts were most likely misaligned when they were pressed together. Aluminum chips were formed when the components were pressed together, indicating that the interference was excessive.

The third round of manufacturing reaction wheels produced usable components. The parts were cut similarly to the previous attempt, except that the central holes were expanded until the motor shafts could be pushed into them by hand. Due to this fit causing much less interference, it was predicted that the wheels may slip if they were just held on by friction and a set screw on a cylindrical shaft. The actions 
outlined in Section 7.3 were taken to ensure that the reaction wheels stayed tightly attached to the motor shafts.

There was more to the chamfering process than predicted. On the first attempt the chamfer width was set to the desired value, the g-code was generated, and the program run on the $\mathrm{CNC}$ machine. When the operation finished, the chamfer came out not only much larger than anticipated, but also had a small flat area. It was learned from this attempt that no chamfer mill is ever actually conical. The tips tend to see high stresses during cutting which causes them to break off or wear. Figure 7.9 shows HSMWork's representation of chamfer mills. Note that the tip is flat and the diameter of this flat spot adjustable to match the wear of the cutter. When the tool's length was probed the probe actually measured the length of the cutter to this flat area. This caused the cutter's path to be lower than anticipated which in turn caused it to take off more material.

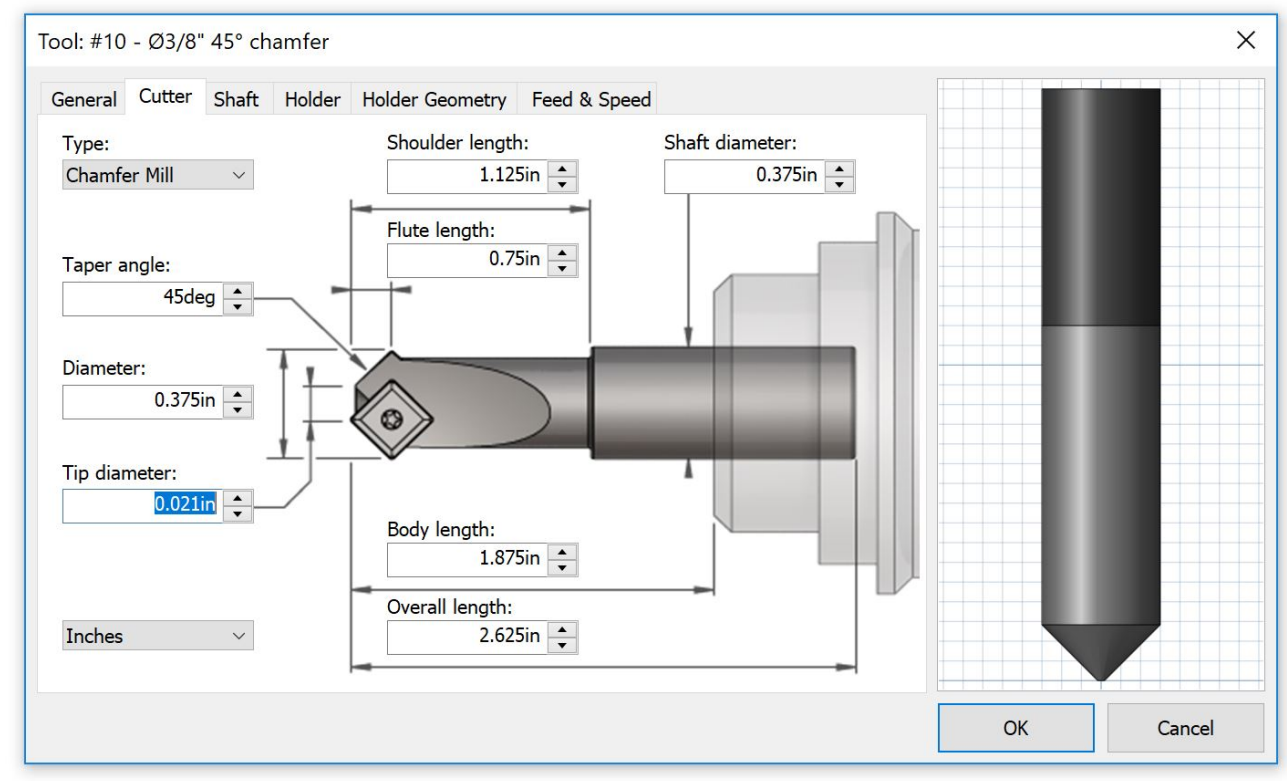

Figure 7.9: Screenshot of HSMWork's representation of a chamfer mill.

To compensate for tip wear, incremental chamfering tests were run on a scrap block. The tip diameter was decreased with each test until the resulting chamfer 
was deemed acceptable. The width of the chamfer was not adjusted to match the prescribed width; it was tuned for functionality and aesthetic appearance.

Another lesson learned concerned cutting inner corner features. Due to the wear of the square end mills, the inner corners of parts did not turn out to have zero radii. For the most part this did not matter. However, the radii of the perimeter of the blocks on the master panels required post machining. Additionally, the radii of the fixtures inhibited the work pieces to sit flush with the datum features.

The radii on the bases of the master panel blocks, which are highlighted in Figure 7.10, caused issues when the minor panels were mated to them. As the screws were tightened the datum surfaces of the minor panels moved closer to datum B, forcing the bottoms of the minor panels to move away from datum A. This caused unwanted gaps between the master and the minor panels. This issue was solved by chamfering the sharp corners of the minor panels.

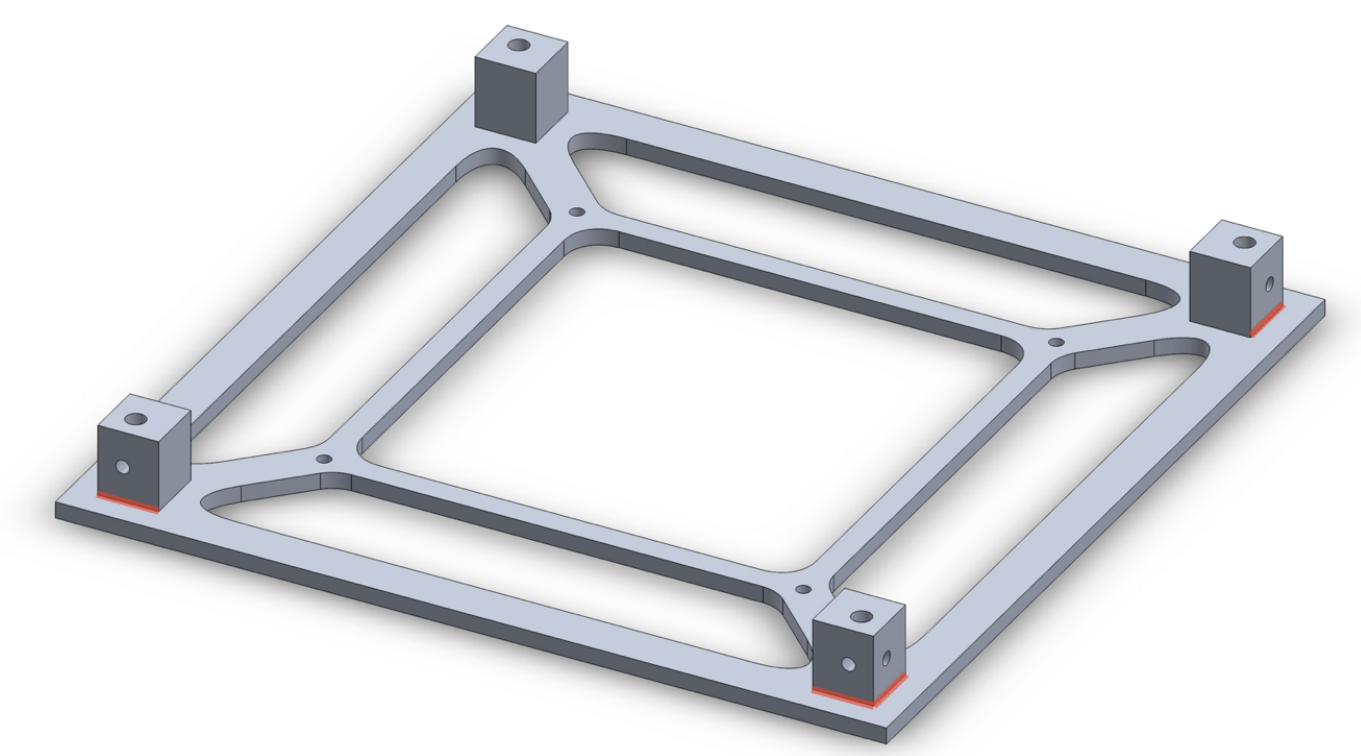

Figure 7.10: Location of undesired internal radii on master panel are highlighted in red. 
This issue also emerged when the panels were attached to the side panel fixture described in Section 7.2.5. As the panels were screwed down onto the plate, they moved away from datums $\mathrm{B}$ and $\mathrm{C}$ due to corner radii in the fixture. For this reason trench features were cut into the fixture, extending the datum B and C surfaces past datum A. A visual explanation is presented in Figure 7.11. Figures 7.7 and 7.8 show these features.
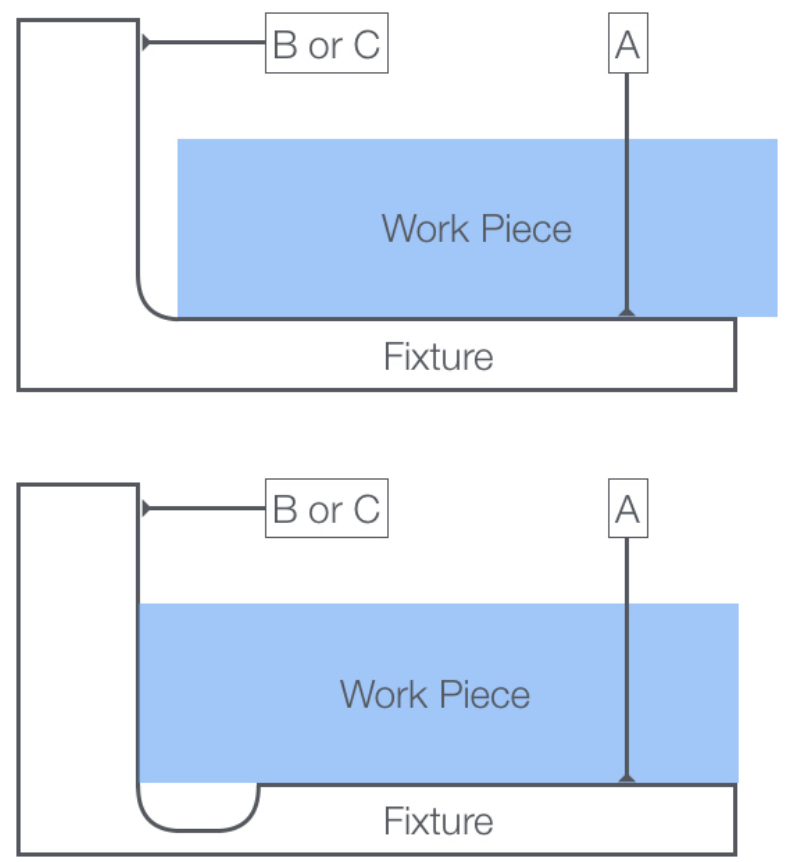

Figure 7.11: Graphical explanation of the issue of internal radii in the side panel and the solution to the problem.

One issue which emerged during the CNC process was the faulty datum structure of the reaction wheel fixture. See Figure 7.5 for reference. Datum A worked well but datum $\mathrm{B}$ and $\mathrm{C}$ were inconsistent. The inaccurate assumption that caused this faultiness was that the two fixtures would be parallel and therefore datum B would be parallel to the $\mathrm{x}$ axis of the machine while datum $\mathrm{C}$ would inherently be perpendicular to it. In reality, the half of the fixture in question was attached to the moving part of the vise. This element has some ability to rotate about the $\mathrm{z}$ axis of the machine (perpendicular to the plane of the reaction wheel) and translate in the $\mathrm{x}$ direction. 
This caused the fixture to be able to rotate around the reaction wheel and therefore the normal vectors of datums $\mathrm{B}$ and $\mathrm{C}$ were in fact not in line with the axes of the mill. Since the fixture had a rotational degree of freedom about the reaction wheel, the areas where the fixture was probed were inconsistent distances away from the reaction wheel center. This in turn caused the chamfers to be offset. It was noticed after the last set of wheels were machined and deemed insignificant enough to not be changed.

\subsection{Wheel Stack Assembly}

To ensure that the wheels were stiffly mated to the motor shafts, the two components were press fitted together and a set screw was tightened onto the shaft.

As described in Section 7.2.2, the hole of the reaction wheel was cut with an end mill and then its diameter was incrementally increased until a motor shaft could be pushed into the hole by hand.

For cinching the screw onto the motor shaft, extra steps had to be taken to make sure that the shaft could not rotate under the set screw and that the set screw did not become loose as a result of vibrations of the wheel. In order to make sure that the cylindrical shaft could not rotate under the screw, a small portion of material was removed from the shaft using a dremel. The resulting flat spot provided a suitable surface for the flat end of the set screw to contact the shaft. 


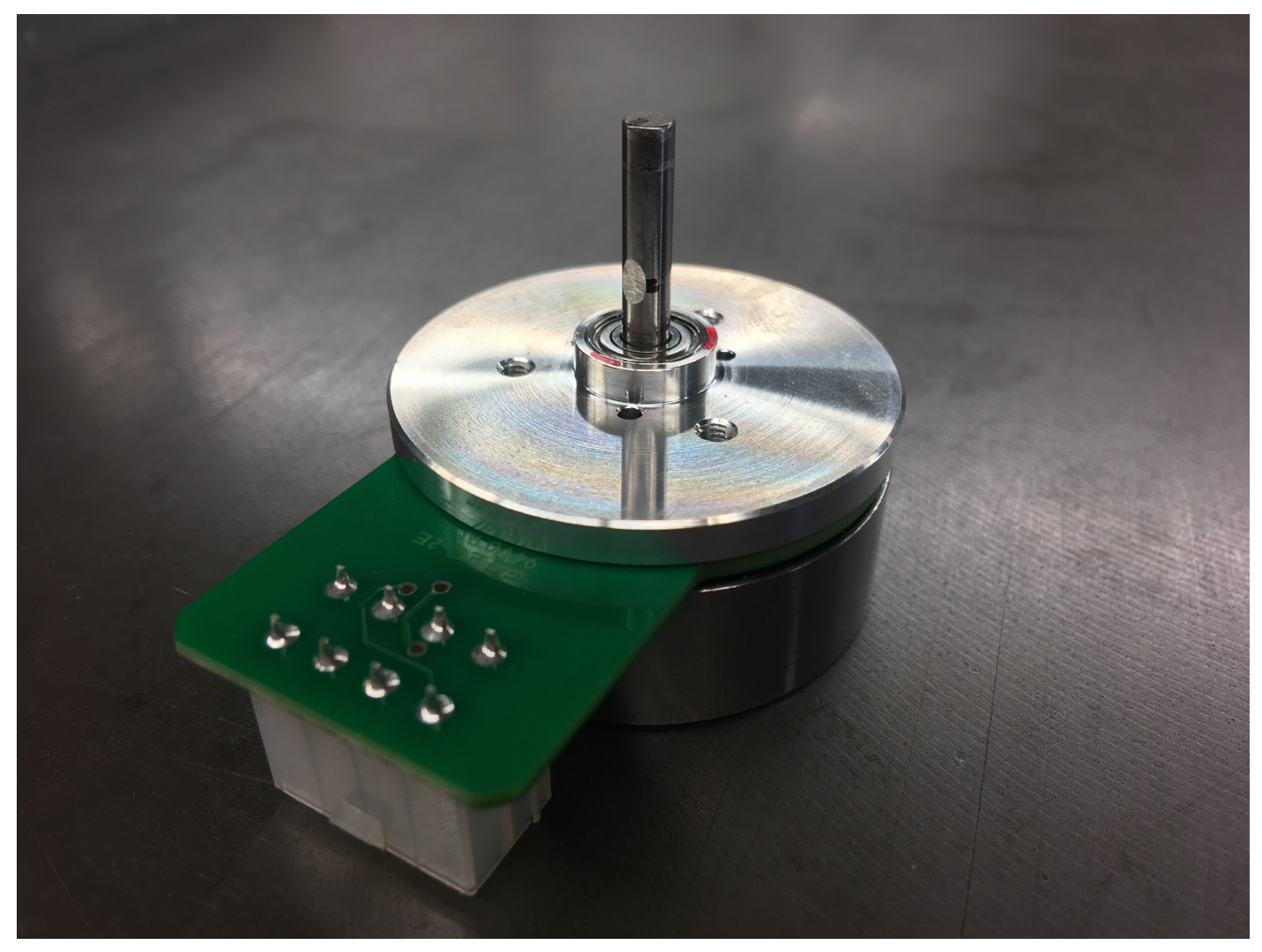

Figure 7.12: Picture of the motor shaft following the modification done for the set screw.

After some rudimentary testing, it was found that the set screws would wiggle themselves free under oscillating torque applications. To mitigate this issue, the set screws were tightened to the shaft and adhered to the threads of the mounting hubs using resin epoxy. 


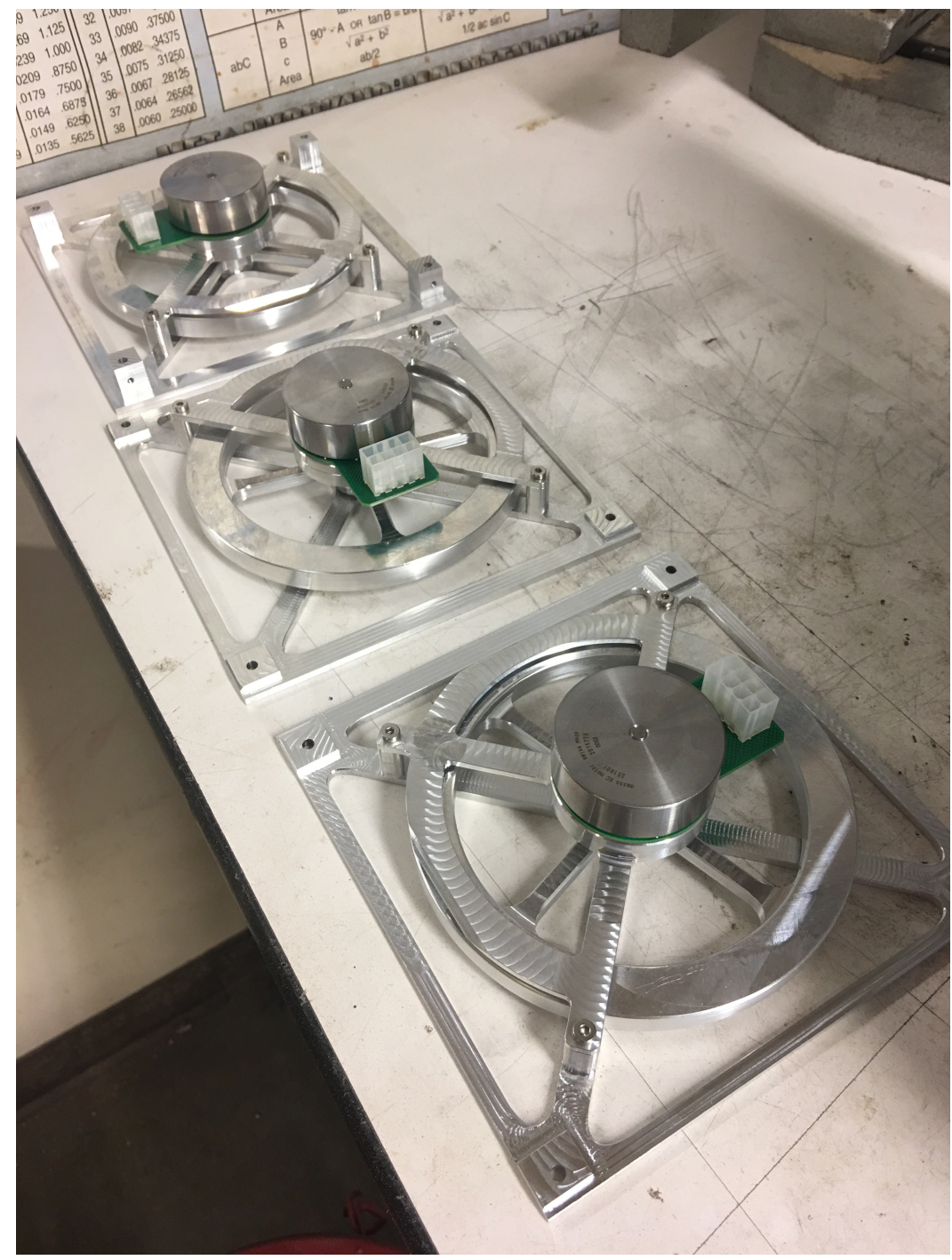

Figure 7.13: Finished wheel stack assemblies.

\subsection{Acrylic Panels}

The acrylic panels were cut from a plate with a laser cutter. The cutter was set up to move slowly while outputting a large amount of power for optimal cutting performance. 


\subsection{Cables}

The electronics within the cube were connected to each other using a combination of connectors and soldering mates.

The three ADC boards and the IMU were hooked up in parallel - power and ground lines were shared along with the data (SDA) and clock (SCL) lines of the I2C bus. Each of the ADC boards had a wire soldered into the A0 channel input. The other end of these wires were crimped into a connector which was clipped into a motor driver's analog output plug. A signal reference ground was also crimped into the same connector and plugged into the ground plane of the Raspberry Pi/PWM hat.

The power harness was constructed with several 22 AWG wires in parallel. Power was fed into the cube from an external power supply with a set of large diameter cables. Each of these connected to six smaller cables. Three pairs of two cables were routed to each of the motor drivers. They were run in parallel to avoid heat

damage from large currents. The two wires were then joined together and fed into a connector. 
Chapter 8

SOFTWARE AND MECHATRONIC IMPLEMENTATION

\subsection{Software Overview}

The software was created in the Simulink environment. The package "Simulink Support for Raspberry Pi Hardware" was used. It enables Simulink programs to be run on Rasperry Pi hardware.

The add-on package includes several blocks designed for operation of Simulink on Rasperry Pi hardware. The most commonly used ones in this program were the I2C Write, I2C Read, and GPIO Write blocks.

Finished programs were compiled by Simulink on the host computer, sent via WiFi to the Raspberry Pi and executed on the Pi. During the operation, the Raspberry Pi intermittently sent collected data to the host computer which displayed the information using blocks such as Scope or Display or saved the data using the To Workspace block.

Simulink is capable of running code at real time on the hardware. For the software of the cube, the program was run at a $100 \mathrm{~Hz}$ frequency since the IMU updates at this frequency.

\subsection{Use of Simulink Stateflow for Sequential Program Execution}

The process of balancing the cube consists of several sequential steps. These steps were implemented in Simulink with a stateflow diagram with time-based transitions. The stateflow diagram in Figure 8.1 illustrates the states and the transitions between them. To summarize, the RESET state resets the IMU and the PWM board. 
SETCAL populates the calibration value registers of the IMU, sets the units of the gyrometer, and configures the output registers of the ADC boards. CONFIG sets the mode of the IMU to NDOF which causes the chip to start measuring and to lock in the calibration values. STRESULTS displays self test results of the IMU. When $C O N T R O L$ is running, the cube's reaction wheels are actively torquing to keep the cube upright. STOPMOTORS shuts off the motors before the program finishes.

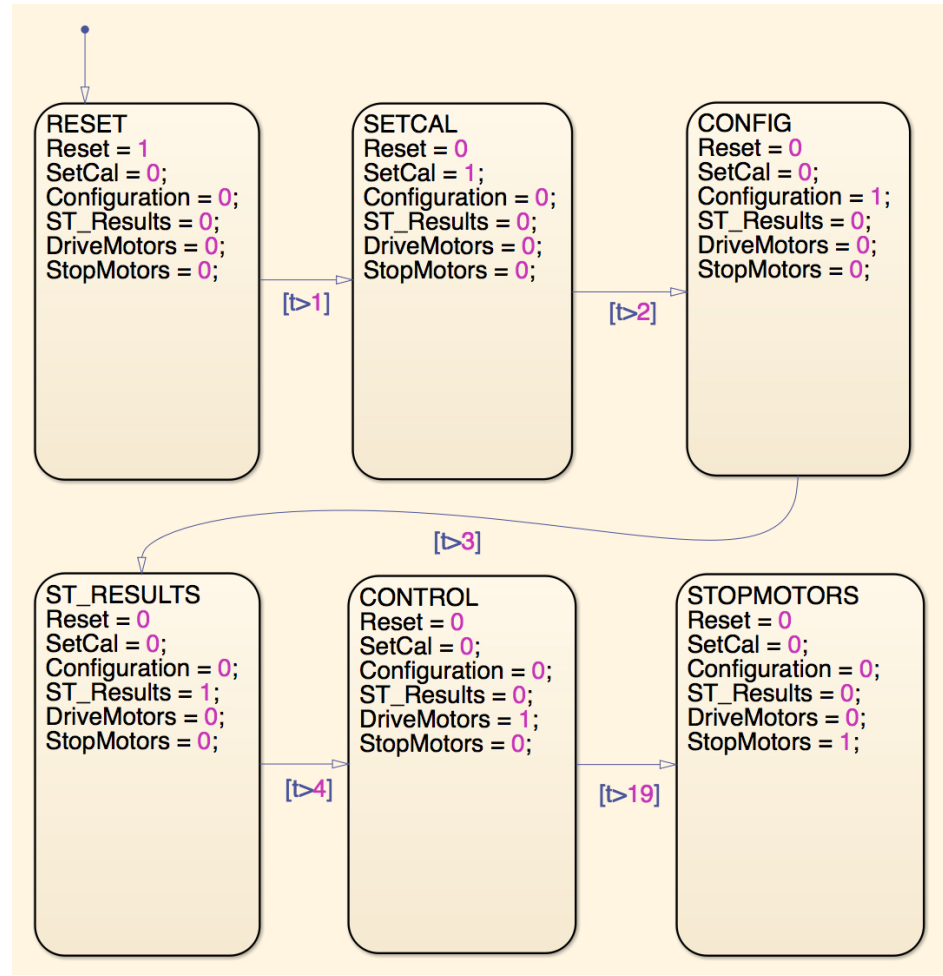

Figure 8.1: Stateflow diagram of the software running on the Raspberry Pi.

When the software transitions into a state, one of the six output variables - Reset, SetCal, Configuration, ST-Results, DriveMotors, and StopMotors - is set high while the others are set low. The value of each of these variables sets a signal line high or low. These signal lines were connected to the enabled subsystems which contain the code associated with the variable. For example, when the software transitions into the CONTROL box of the stateflow model, the DriveMotors variable is set high and as a result the code to run the control algorithm is run while the rest of the code is 
not executed.

All of the states except for CONTROL and STOPMOTORS simply read and/or write a few registers and are therefore not discussed. The CONTROL and STOPMOTORS are explained thoroughly in Sections 8.3.1 and 8.3.2.

\subsection{Control Software}

The internals of the CONTROL task are shown in Figure 8.2. The subsystem on the right is composed of mechanisms to actuate the reaction wheels given a certain torque value and to sense the orientation and rotational rates of the cube and the rotational rates of the wheels. On the left of the graphic are the mathematical operations which constitute the state estimator and control gain matrix. These blocks were copied directly from the simulation.

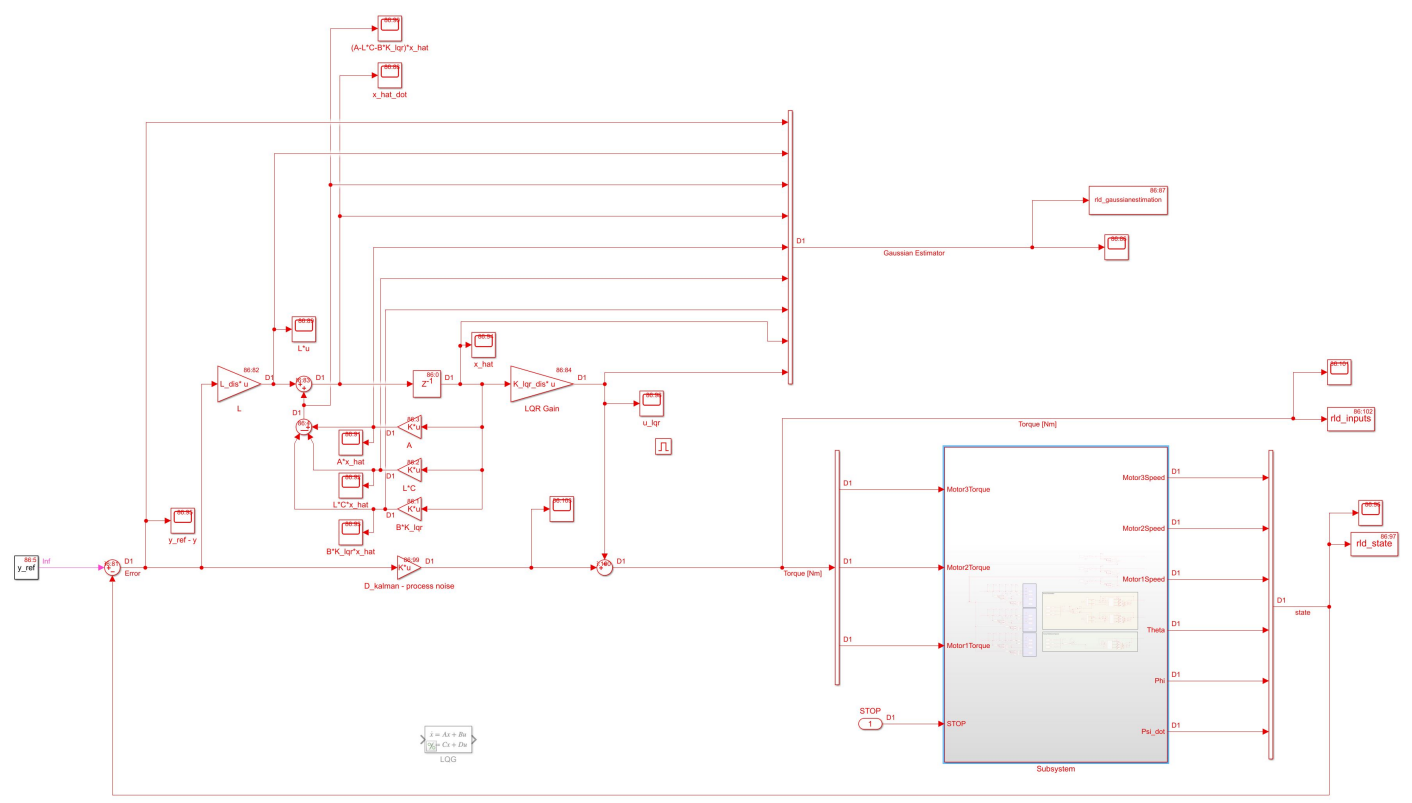

Figure 8.2: Simulink model of the control algorithm running on the cube. The subsystem contains the programs for actuation and sensing. 


\subsubsection{Motor Drivers}

The motors are commanded by the motor driver electronics. The drivers were set up using ESCON Studio, a Maxon-made software package which is capable of configuring the motor drivers and tuning them for closed-loop control.

Using ESCON Studio, the drivers were configured to operate as closed-loop current controllers. The digital input connector was set up to take a PWM signal which was decoded into a current, a signal declaring the direction of the current, an enable signal and a ground line. The analog output connector was set to output the current speed of the motor.

The Simulink block diagram which commands the motor drivers to actuate the reaction wheels is shown in 8.3 below.

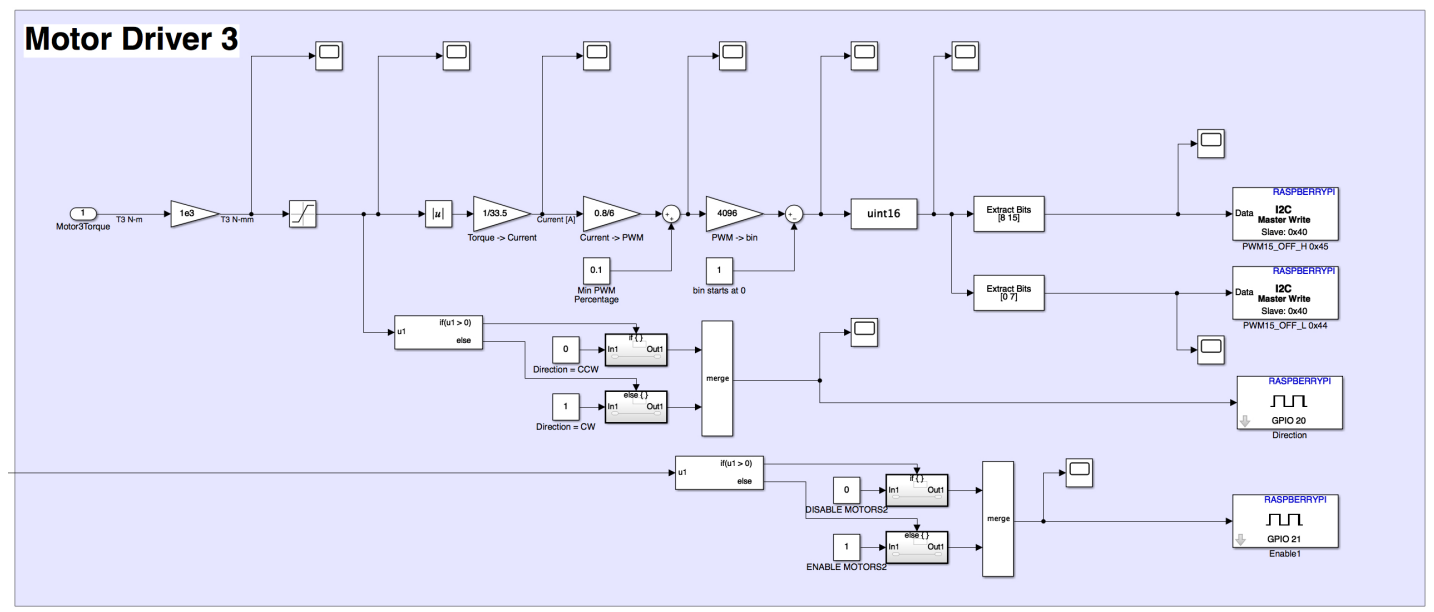

Figure 8.3: The portion of the program which commands the motor drivers based on a torque input.

The commanded torque value gets passed into the block diagram from the left. This signal is first converted from Nm to Nmm using a simple gain. It is then saturated to a physically reasonable limit to avoid the motor from torquing detrimentally hard in the case of a non-functional control logic. Next, the sign of the value is interpreted using an if/else logic and the direction pin set accordingly. The torque constant gain 
transforms the torque value into a current value. Then, based on $10 \%$ duty cycle having been set in ESCON Studio to result in 0 A of current and $90 \%$ translating to $6 \mathrm{~A}$, the driver software calculates the PWM duty cycle. This value is then changed to a 16 bit binary signal, split, and sent to the appropriate registers of the PWM generator board. This board sets the PWM outputs accordingly and commands the motor drivers to actuate the motors with a specific torque and in the correct direction.

Additionally, the enable GPIO pin's signal remains high as long as the EMERSTOP signal is low. If at any time the control software decides that an emergency shutoff is necessary, the EMERSTOP flips high and the motors are deactivated.

\subsubsection{Reading Motor Speeds}

The motor drivers were set up to output the current speed of the motors with $0 \mathrm{~V}$ representing -1000 rpm and 4V translating to $1000 \mathrm{rpm}$.

The output lines were initially fed from the motor drivers to one ADC board which would translate the signal to a digital voltage and populate a register with the most recently calculated value. The process of reading the speed of one of the motors required two steps. First, the configuration register of the ADC board had to be written to. Three bits of this register controlled which of the available analog channels the ADC board would translate and write to the output register. The second step involved reading this output register. It was therefore of importance to do these two tasks sequentially and right after one other to avoid other tasks from setting the configuration register differently before the speed could be read.

Since Simulink automatically handles multitasking and has a tendency to start with the I2CREAD instructions and end with the I2CWRITE instructions, it gener-

ally did not process the two requests sequentially. In fact, it would read the output register three times without the $\mathrm{ADC}$ having been commanded to write the digital 
voltage value of another analog line to that register. Then, towards the end of the multitasking sequence, Simulink would write to the configuration register three times in a row.

One attempt to fixing this problem consisted of putting the write and read operations into different states. It was predicted that the control loop would be executed, and that the three motor's write and read functions would execute so quickly that the control logic could run again at the next time step. However, it was found that each of the states executed at the discrete time steps. The software would enter into the Control state, run the tasks in it, step to the WriteMotor3 state, run the tasks inside of this state at the next time step, and so on and so forth. Therefore, the control loop would execute only every seven time steps which conflicted with the discrete control algorithm designed to run at every time step, as explained in Chapter 9 .

A clean solution to this problem was not found. Instead, two more ADC boards were added to the cube and only one channel of each board was used. Each of the boards was then configured to always output the translated value of one channel during the CONFIG state. During the remainder of the program, the configuration register did not have to be written to anymore.

The Simulink blocks representing the write and read tasks are shown in Figures 8.4 and 8.5 , respectively.

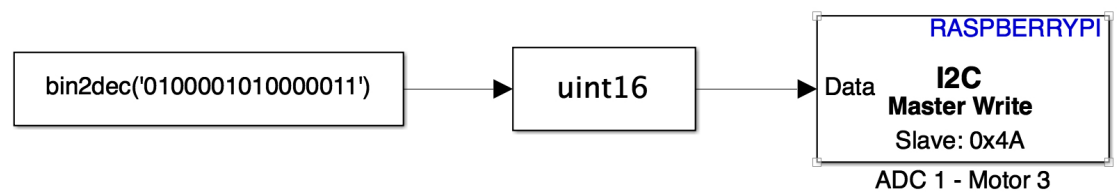

Figure 8.4: The $\mathrm{I}^{2} \mathrm{C}$ write function which commands the ADC board to save its channel 0 translation results in the output register. 


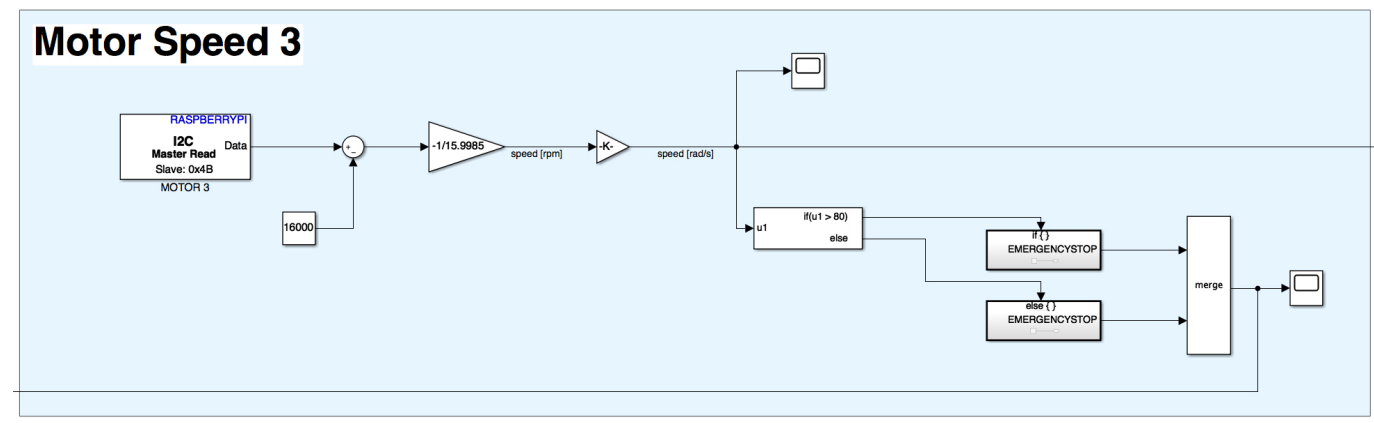

Figure 8.5: The $\mathrm{I}^{2} \mathrm{C}$ read function which reads from the output register of the ADC.

The conversion from the binary value read from the ADC board to the rotational speed of the wheels in rpm was found experimentally. First, one of the motor drivers was configured to employ a closed-loop speed controller. Using this driver, a motor was spun up to a constant speed and the binary value was recorded. A handful of data points were taken and a linear relationship found.

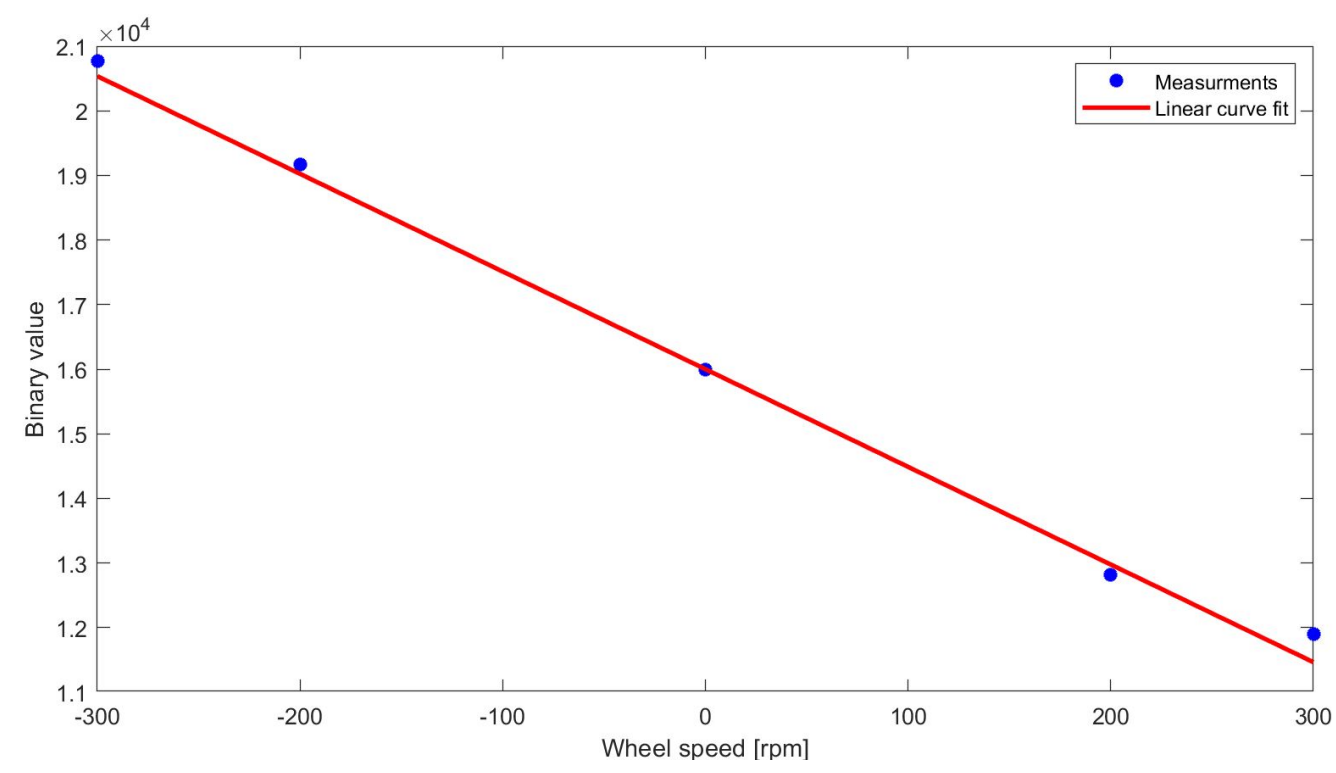

Figure 8.6: Binary values outputted by the ADC board representing reaction wheel speeds. 


\subsubsection{Sensing Orientation}

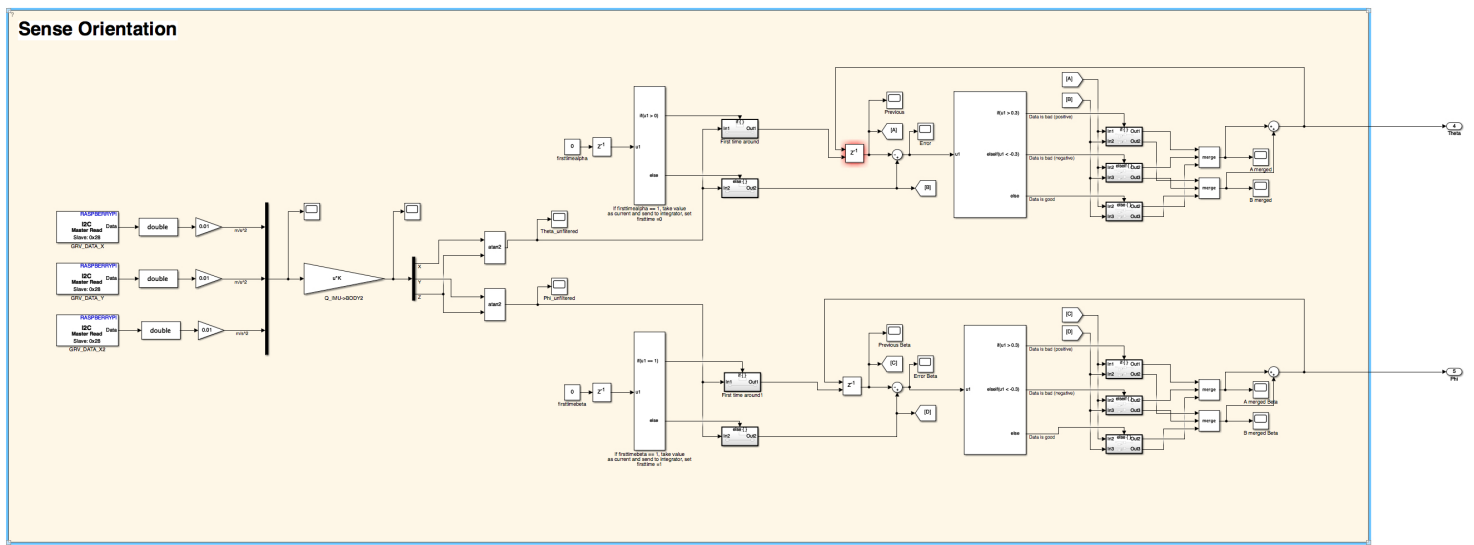

Figure 8.7: Portion of the program which senses the orientation of the cube, derives the angles $\theta_{\text {cube }}$ and $\phi_{c u b e}$, and post-processes the data.

In the simulation the orientation was output as a quaternion and the angles $\theta_{\text {cube }}$ and $\phi_{\text {cube }}$ were derived from it. In the case of the real hardware, this process was simplified due to the capability of the IMU to output the gravity vector $r_{g, S E N S O R}$ directly. This vector was then expressed in the $C S Y S_{B O D Y}$ frame using the coordinate system transformation matrix $Q_{S E N S O R->B O D Y}$. Then, the angles between the $-\mathrm{z}$ axis of $C S Y S_{B O D Y}$ and $r_{g, B O D Y}$ in the $\mathrm{x}-\mathrm{z}$ and $\mathrm{y}-\mathrm{z}$ planes were measured and declared as $\theta_{\text {cube }}$ and $\phi_{\text {cube }}$. The section of the code responsible for this is shown in Figure 8.8.

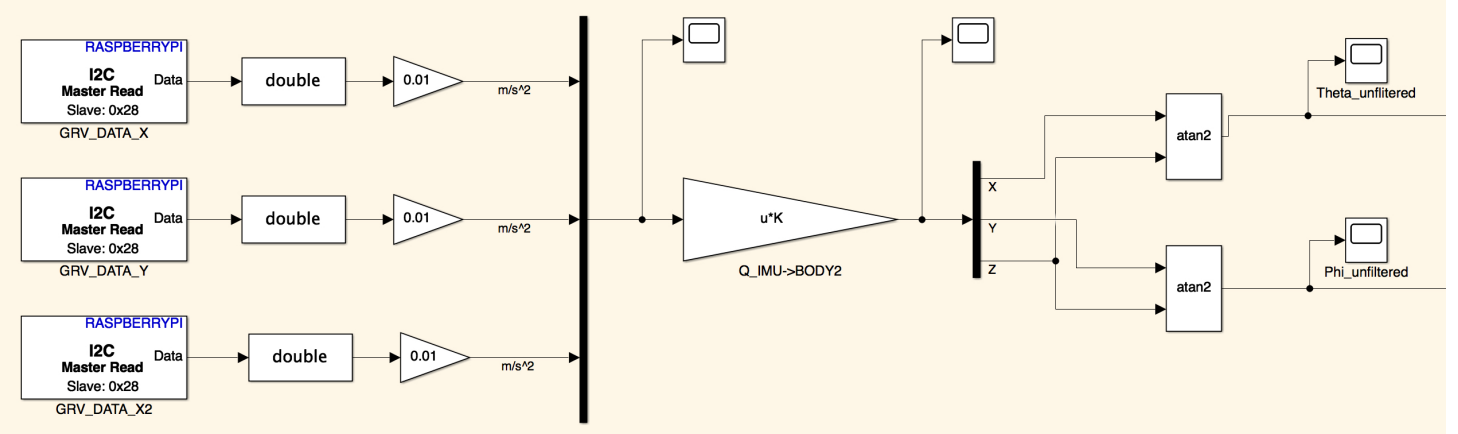

Figure 8.8: Cluster of Simulink blocks which read the orientation and find $\theta_{\text {cube }}$ and $\phi_{\text {cube }}$ based on it. 
One issue with the orientation measurements was the integrity of the signal. An example of the signal is shown in Figure 8.9 .

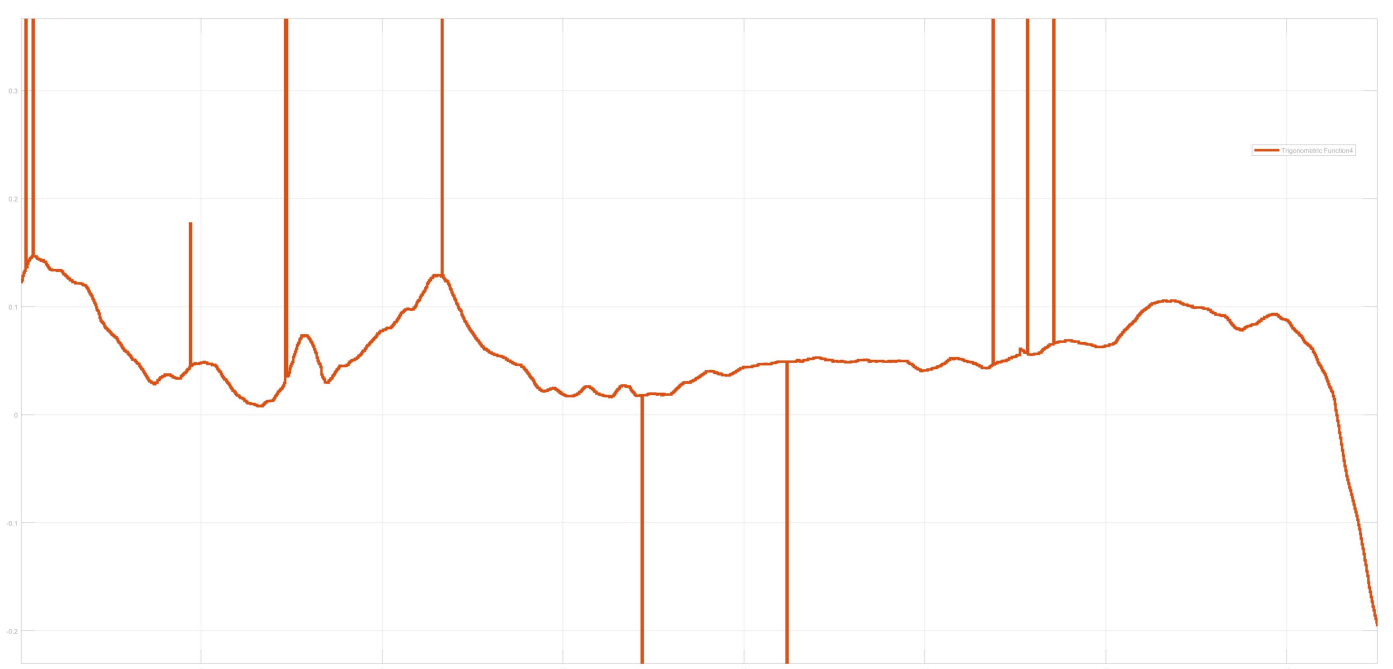

Figure 8.9: Sample signal before it was post processed.

The cause of the issue was not found, though best guesses pointed towards an I2C communication issue. It seemed that during the communication, the first bit of each byte had a chance of being flipped high. This caused the communicated values to jump to a certain value. Another detail pointing at an $\mathrm{I} 2 \mathrm{C}$ issue was the timing of these spikes. The erroneous readings were always one time step long.

To remedy this issue, a filter post-processed the orientation signals. The architecture of the filter is shown in Figure 8.10 below.

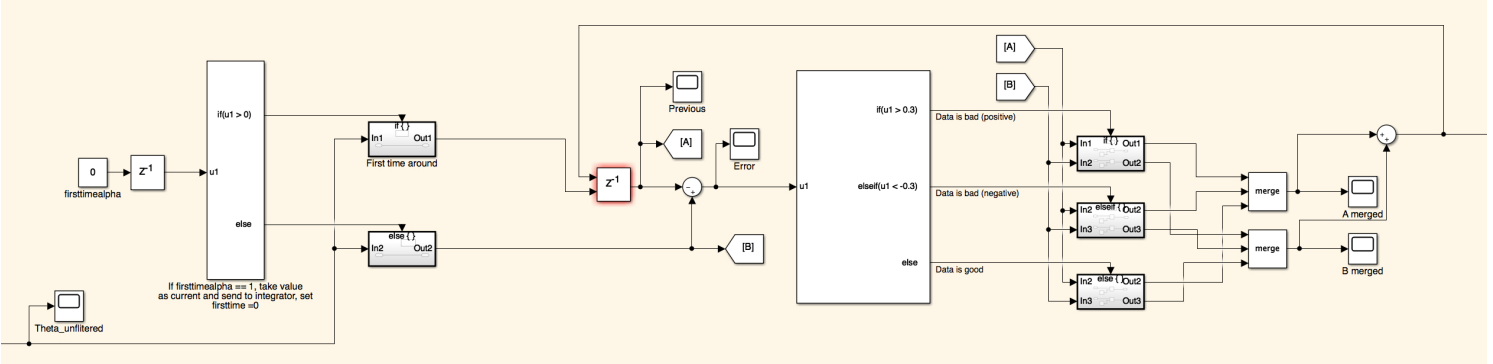

Figure 8.10: Post-processing filter logic. 
The signal enters the filter at the bottom left of Figure 8.10 and the filtered signal is outputted at the right of the illustration. It works on the premise of comparing the current measurement to the previous one. If the absolute value of the difference of the two is larger than a threshold value, the current signal is assumed to be erroneous and therefore overwritten with the previous time step's.

Two if/else functions exist in the filter. The first one, seen on the left hand side of the Simulink model either executes an initial value generator or passes the signal through. The first time this if/else function is run, the generator function measures the current orientation and writes this value to the initial condition field of the delay function further to the right. A combination of a constant and a delay block create a discrete signal which is high during the first time step and low after that. This signal determines the path of action of the first if/else logic.

Once the initial value of the orientation is loaded into the delay block, the filter begins to compare the signal to the previous one. The second if/else block passes the signal through if it is without the bounds. Otherwise it executes one of the two functions which override the signal if it is larger than the positive threshold or smaller than the negative threshold. The result of this filtering can be seen in Figure 8.11. 


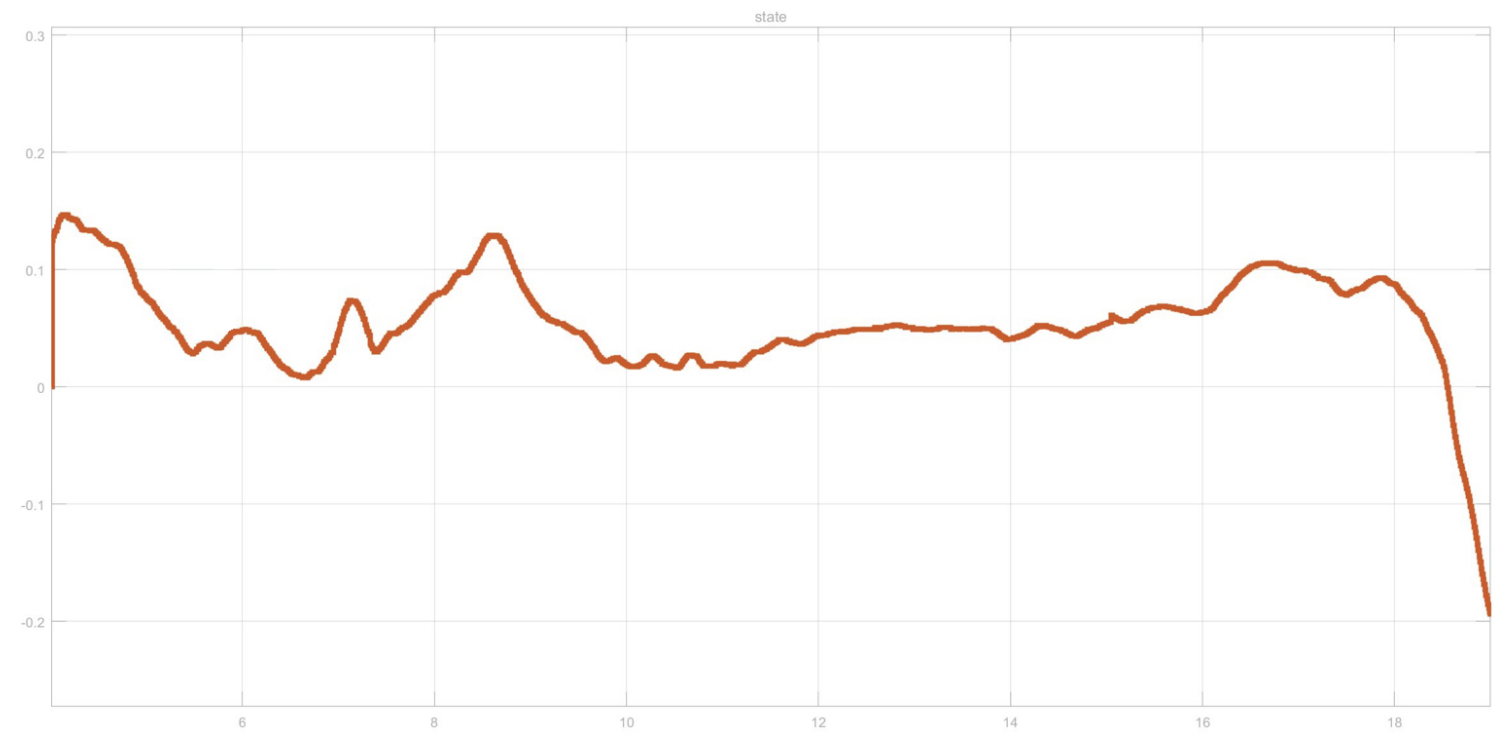

Figure 8.11: Sample signal after it was post processed.

\subsubsection{Sensing Rotational Rates}

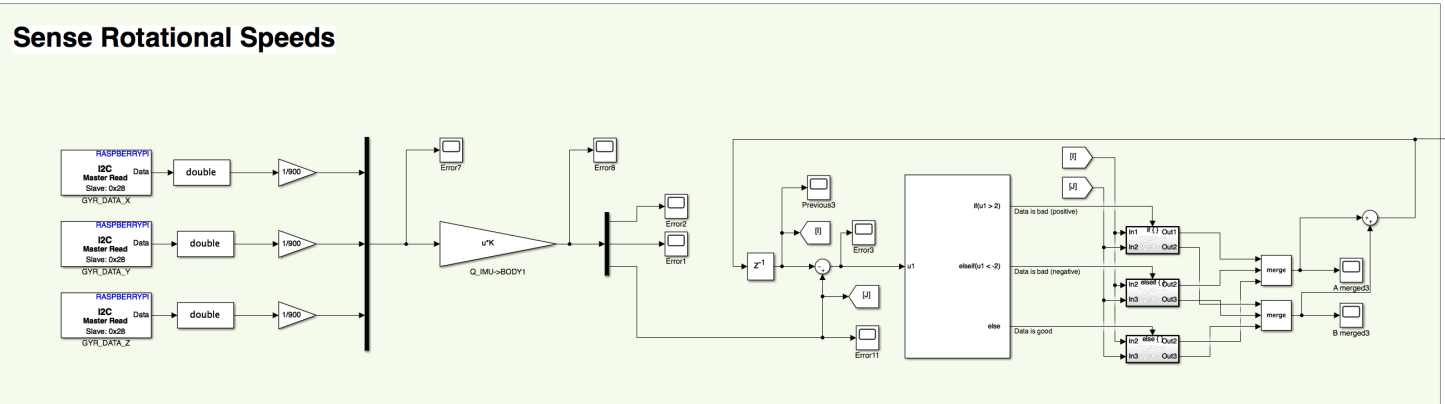

Figure 8.12: Portion of the program responsible for sensing the rotational speed of the cube, converting the readings to $C S Y S_{B O D Y}$, and postprocessing $\dot{\psi}_{\text {cube, } \max }$.

Sensing the rotational rates of the cube was quite straight-forward. The rates about $C S Y S_{S E N S O R}$ were read from the appropriate registers, transformed from binary to integer numbers, multiplied by a gain to adjust for units, measured in $C S Y S_{B O D Y}$ by multiplying them by $Q_{S E N S O R->B O D Y}$, and then $\dot{\psi}_{\text {cube }}$ was pushed through the same post-processing filter applied to the orientation sensing signal, as explained in 
Section 8.3.3. Different threshold values were used for this filter.

\subsection{Emergency Stop}

Abort functionality was developed to keep the mechanism from self-destructing. Two scenarios were protected against - excessive wheel speed and torque values being commanded when the program finished executing.

The reaction wheels were observed to wobble a bit. To make sure that these out-of-plane accelerations would not cause damage to the mechanism, an emergency stop function was integrated into the control algorithm to prevent the wheels from spinning too fast. As can be seen in Figure 8.5, an if/else logic was implemented which set the EMERSTOP line high in the case that the wheel speeds exceeded 80 radians/second. This then caused the motors to be disabled.

When a previous version of the program, which did not have the StopMotors state, finished executing, the motor command written to the PWM generator continued to be sent via PWM to the motor drivers. In response, the motor drivers operated a closed-loop torque control algorithm and accelerated the wheels to dangerous rotational speeds.

To mitigate this issue, the StopMotors state was incorporated in the software. A second before the program is scheduled to finish executing, the EMERSTOP line is set high and the motor drivers stop their control of the motors.

\subsection{Testing}

A series of tests were conducted to verify the functionality and accuracy of all measurement and actuation software. 


\subsubsection{Cube's Orientation}

The first things to be verified were the cube's angles $\theta_{\text {cube }}$ and $\phi_{\text {cube }}$. It was known that when the cube was in the balanced position, both angles should be close to zero. When the cube was first lifted to this position, these values were very far from zero. This problem was discovered to be a result of $C S Y S_{W O R L D}$ of the IMU to be different than that of the simulation. While the simulation used a CSYS with the $-\mathrm{y}$ axis pointing in the direction of gravity by default, the sensor created a CSYS with the $-\mathrm{z}$ axis pointing down.

At first, the sensor's axis mapping was reconfigured. Problems with this system emerged, however, as the mapping did not function as expected. Instead, $C S Y S_{W O R L D}$ of the simulation was changed to match that of the IMU.

The IMU defined CSYS $S_{W O R L D}$ using a combination of the accelerometer and magnetometer. The accelerometer sensed gravity, which defined the direction of the -z axis. The magnetometer found north which was assigned as the second axis of the CSYS. To rotate $C S Y S_{W O R L D}$ in the simulation to match the IMU's CSYS, the assembly was rotated by 90 degrees in SolidWorks and the simulation was rebuilt.

Once this axis reconfiguration was completed, the measurement of angles $\theta_{\text {cube }}$ and $\phi_{c u b e}$ were zero in the balanced position. Next, the sign of them had to be verified. To

do so, the simulation's LQR gain was replaced with a scalar gain of zero, effectively deactivating the torque input. The simulation was then run and the trajectory of the cube observed. The trajectory was then imitated with the real hardware and the changes in $\theta_{\text {cube }}$ and $\phi_{\text {cube }}$ were observed. Measurements from the simulation and the real hardware matched, as can be seen in Figure 8.13, so no further adjustments had to be made. 

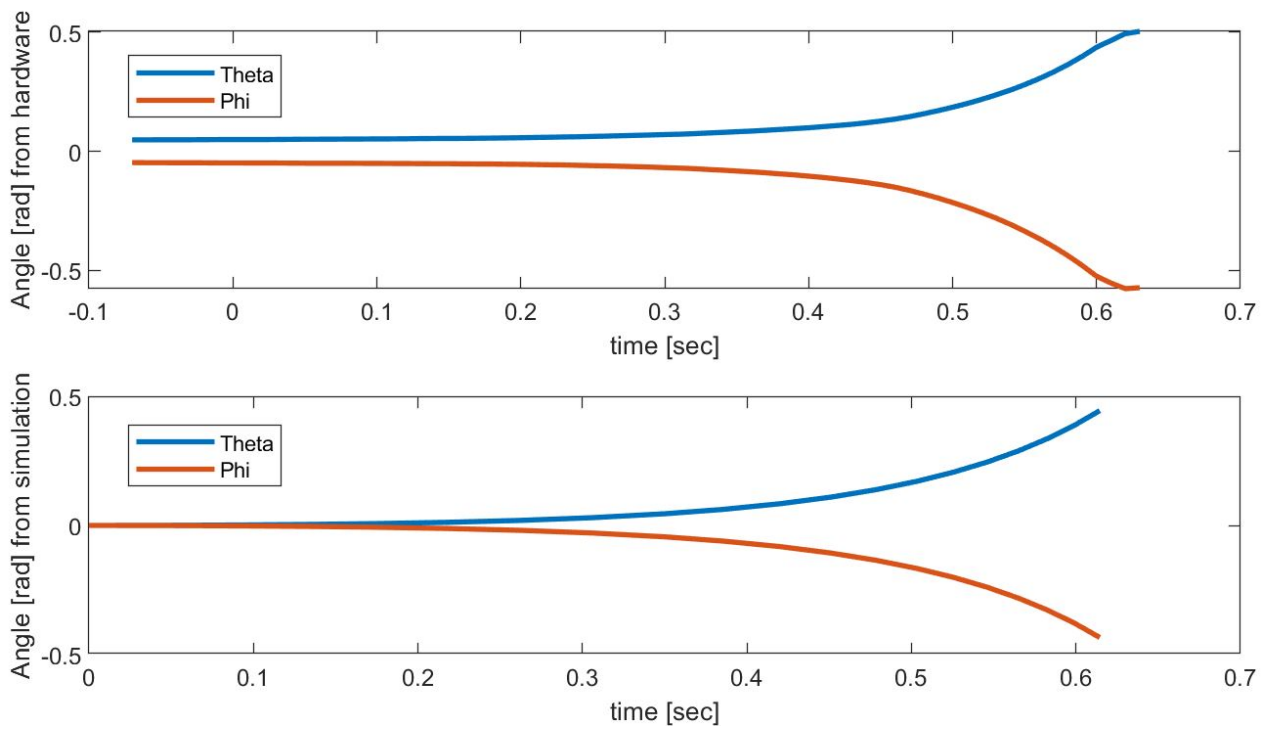

Figure 8.13: The simulated values and hardware measurements of $\theta$ and $\phi$ throughout the same motion. The similarity in the data verified that the sign convention of the angles matched.

\subsubsection{Direction of Angular Rate}

In order to verify the sign of $\dot{\psi}_{\text {cube }}$, the cube had to be spun about the $\mathrm{z}$ axis in the simulation. This was achieved by setting the inputs torque values to the same constant. In theory, this should cause a non-zero net torque about the $\mathrm{z}$ axis. It was predicted that setting these values high would cause the cube's spin rate to have a much smaller time constant than the acceleration due to gravity. When the simulation ran, the cube began to spin in one direction. The motion was observed, imitated with the real hardware, and the results compared. Similarly to the angle measurements, the rotational rate $\dot{\psi}_{\text {cube }}$ had the same sign convention for the simulation and hardware. Therefore, no changes had to be made.

\subsubsection{PWM Generation}

The functionality of the PWM generator board was tested before the wheels were first spun up. With an oscilloscope attached to two one of the PWM channels on 
the board, a simple Simulink model was run on the Raspberry Pi. This program sent appropriate values via $\mathrm{I} 2 \mathrm{C}$ from the Raspberry Pi to the PWM to command it to generate a PWM signal. When this simple test failed, it was discovered that the PCA9685 chip on the PWM hat could be reset via an I2C Read command. After doing so, the PWM wave was generated successfully.

\subsubsection{Wheel Spin Direction}

The directions of the rotational velocities of the wheels were verified by applying constant input torques in the simulation, observing the direction of rotation of the wheels, applying the same torque in the real system, observing the wheel's directions of rotation, and making sure that the two matched. The conditions of the if/else block, which was used to set the direction pin on the motor drivers, was changed to accomplish this.

\subsubsection{Wheel Identification}

From the SimScape simulation, it was not clear which wheel was which. To figure out which wheel was actuated by which torque input and which speed measurement was related to which wheel, two sequential tests were performed. The first one consisted of setting the input torque to one of the wheels while keeping the other two to zero. From the 3D simulation generated by SimScape, it could be observed which wheel started spinning. The second test saw another wheel receiving a non-zero torque command and again observing which wheel was actuated. The motor control software was rearranged to match this convention. 
Chapter 9

\section{ADJUSTMENT OF CONTROL ALGORITHM TO OPERATE ON HARDWARE}

\subsection{Process}

The simulation of the LQR controller and state estimator used a continuous time model of the mechanical system as the plant. The actual hardware did not work in the same way since the sensors and motor drivers operated in a discrete manner. For many electronics in this system, such as the processor, ADC board, and motor drivers, the sampling and calculation increments could have been decreased so much so that a control logic designed for a continuous system may have worked. However the IMU, which calculates the gravity vector from a combination of accelerometer, gyrometer, and magentometer readings while also filtering the response, operated at $100 \mathrm{~Hz}$. This was therefore the bottleneck of the system. Therefore, to develop a sound control algorithm which was to be implemented in the hardware the control gains had to be adjusted.

To optimize the control algorithm for this discrete system, several elements of the simulation and gain mathematics had to be altered. All blocks in the simulation had to be changed to their discrete counterparts, the solver settings had to be modified, and the controller and state estimator gains needed adjustments.

Switching the simulation from continuous to discrete required the modification of the plant model. The Simscape model did not function with a fixed time step solver. Therefore, it had to be replaced with a discrete state space block. The matrices representing the linear, discrete system had to be calculated for this block. Using the command $C 2 D$, which stands for continuous to discrete, a time step input argument of 0.01 seconds, and the continuous state space representation, the discrete state space 
matrices were calculated. These matrices were fed to the discrete state space block which represented the system's dynamics. The matrices representing the discrete state space system were approximately two orders of magnitude smaller than their continuous counterparts, as measured by the norm. Theoretically the matrices of the discrete system should approach the ones of the continuous system as the time step is decreased.

The delay block had to be replaced as well. The one to take its place was a discrete delay block. This element works at a fixed time step, the parameter of which can either be edited manually or made to be inherited from the solver settings. In this scenario, the blocks was commanded to take the solver settings.

Both the LQR and state estimator gains had to be adjusted to work for the discrete system as well. This was accomplished by duplicating the lqr and kalman commands, but instead feeding them the discrete state space representation of the cube.

One final adjustment to the simulation was made to the solver settings. Instead of running in continous mode, or as Simulink calls it 'variable time step' with automatically set time steps, the simulation was changed to work with fixed time steps. The time step was modified from 'auto' to $0.01 \mathrm{~s}$ to match that of the real system.

Finally, after modifying the simulation and mathematics which calculated the gain matrices, the gains were pushed to the software running on the cube.

\subsection{Results}

Overall, the discrete controller worked well in the simulation. It achieved its goals of driving all states to zero in a timely manner while keeping the reaction wheel speeds low. 
Two major difference were found between the simulation of a continuously operating algorithm on a nonlinear system and the simulation of a discrete control logic driving the reaction wheels of a linearized system.

The first difference can be attributed to the linearization of the plant. While both systems were started with zero initial conditions, the linear system did not naturally diverge from its upright position. The nonlinear system's center of gravity was not above the bottom corner when it was in its upright position. Therefore when the simulation began with the system in its upright position, there existed an initial moment about the bottom corner which caused the cube to begin to fall over. As a results, the reaction wheels were spun up and the act of balancing the device began. In the linear system this was not the case. Starting the simulation with the cube in the upright position did not cause the cube to fall over. Therefore, no input was required and the system was unrealistically stable in an unstable position. To mitigate this issues, the initial conditions of the linear state space block representing the system was modified. A value of 0.01 was entered and the simulation was run. The resulting discrepancy in the motion of the system between the two simulations can be seen in Figures 10.3 in Chapter 10 and Figure 9.1 .

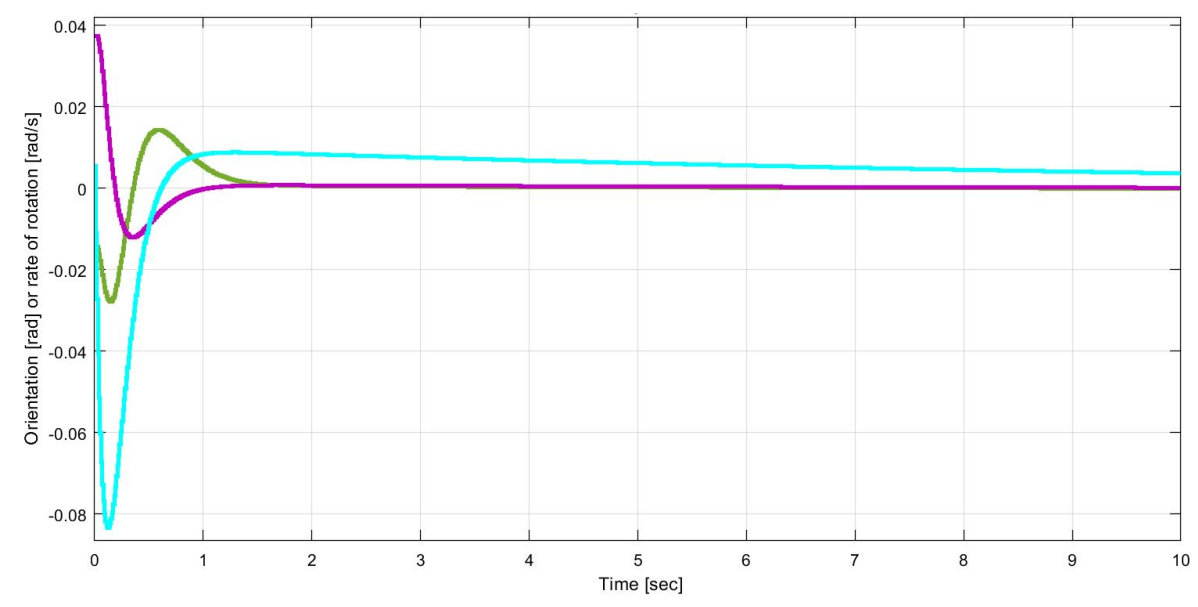

Figure 9.1: Cube orientations $\theta$ and $\phi$ and rate of rotation $\dot{\psi}$ as calculated by the discrete simulation with a linearized plant model. 
This inherent stability in the upright position rather than the balanced position of the linearized system also caused all of the states to be able to converge to zero, which was not the case for the nonlinear simulation. The reason for this behavior of the nonlinear system is described in detail in Section 10.2.1. Figures 9.1 and 9.2 show the two cube angles, the cube's rotational rate, and the three reaction wheel speeds to converge to zero.

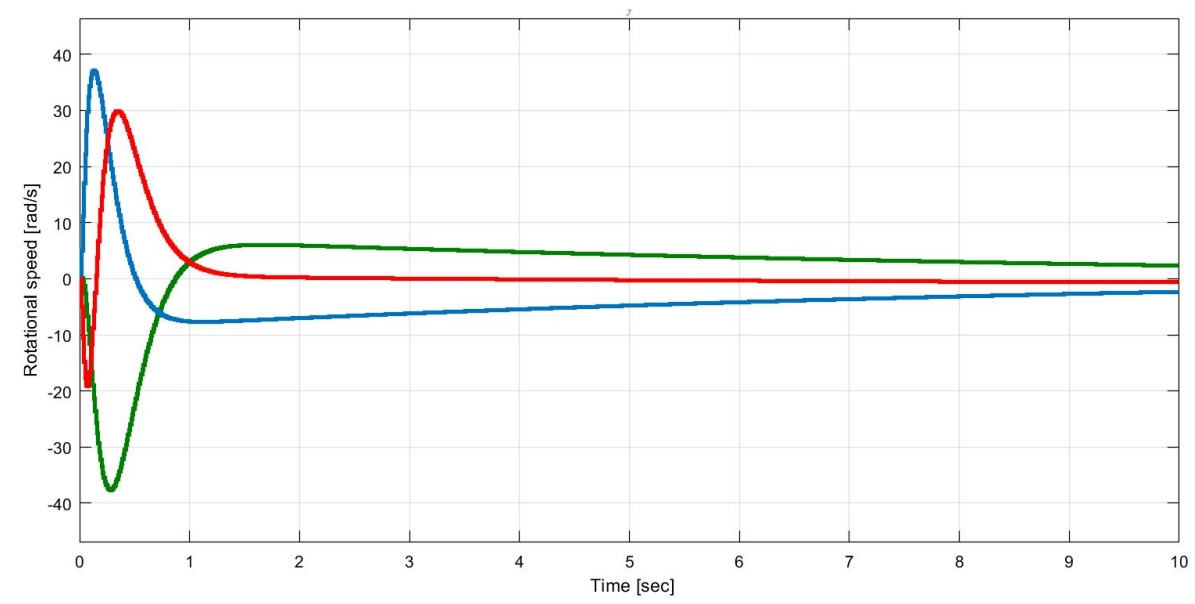

Figure 9.2: Reaction wheel speeds as calculated by the discrete simulation with a linearized plant model. 
Chapter 10

RESULTS

The results of this thesis are split into several sections. First, the accuracy of the dynamics simulation is investigated. Then, the functionality of the control algorithm in the simulation and furthermore in the real hardware is described. Lastly, suggestions for continuing this project are listed.

\subsection{Simulation Analysis}

The accuracy of the dynamics simulation was verified by comparing the mechanical parts' masses and inertias to the actual hardware. The reaction wheels' inertia was found by spinning the wheel with a preset torque and observing the angular acceleration. The cube's inertia was not directly measured. Instead, the motion of the cube as it fell from its balanced position was compared to that of the simulation. Masses of parts were measured using a scale.

The mass property comparisons yielded that the simulation is quite accurate. It was concluded that the simulation is a valid representation of the hardware.

\subsubsection{Reaction Wheel Mass Properties}

To find the inertia of the reaction wheels, the wheels were accelerated with a known torque and their rotational speed was measured. This was achieved by using the Simulink code for driving and measuring the speed of the motors as described in Chapter 8 but with a constant torque input of $75 \mathrm{mNm}$. Figure 10.1 shows the result of this testing. A best-fit line was created for the data, the slope of which was the angular acceleration of the wheels. Then, the result was divided by the known input 
torque to find the rotational inertia.

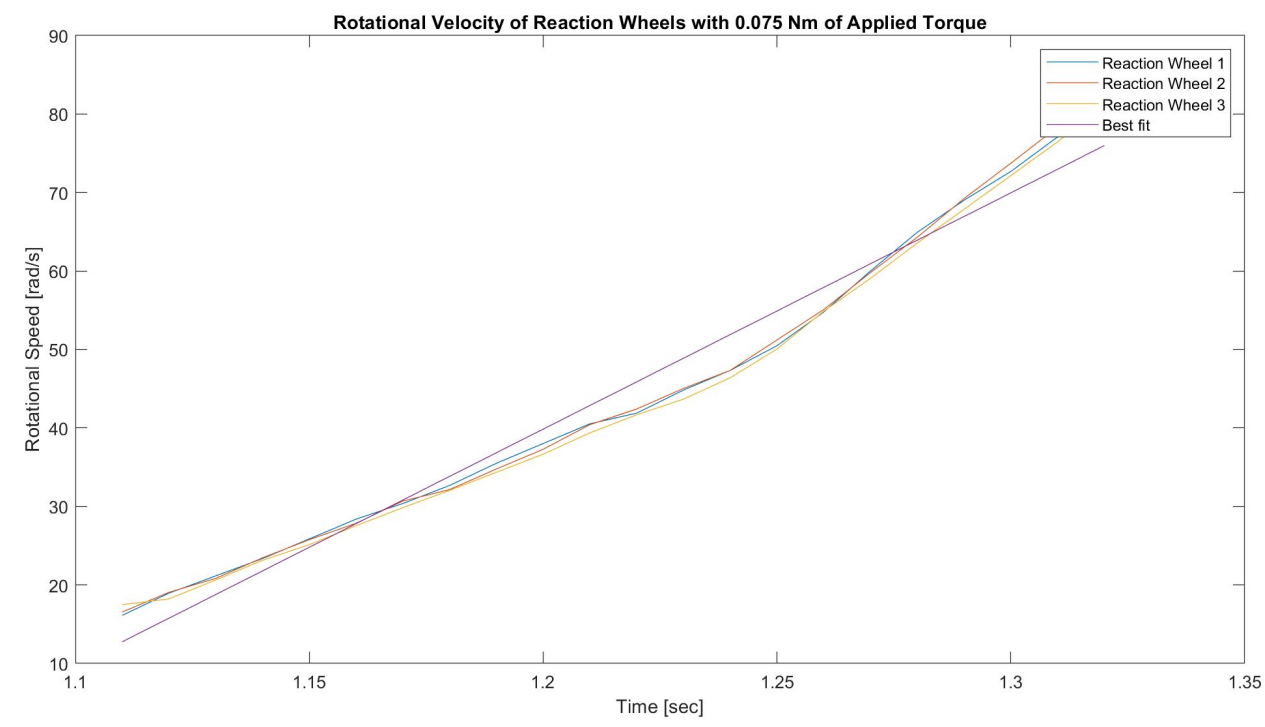

Figure 10.1: Given a known input torque, the angular velocities of the reaction wheels were measured to find their inertias.

The results of the mass property measurements are given below in Table 10.1 .

\begin{tabular}{c|c|c|c} 
Mass property & Simulation value & Hardware value & Percent difference \\
Mass & $118.75 \mathrm{~g}$ & $119 \mathrm{~g}$ & $0.21 \%$ \\
Inertia & $3.05 * 10^{5} \mathrm{~g}-\mathrm{mm}^{2}$ & $2.49 * 10^{5} \mathrm{~g}-\mathrm{mm}^{2}$ & $20.12 \%$
\end{tabular}

Table 10.1: Comparison of mass properties of reaction wheels used in simulation and measured from hardware.

Deducing from the data presented in Figure 10.1 and Table10.1, the reaction wheel is fairly well presented in the simulation. The discrepancy of the inertia measurement is likely a consequence of nonlinearities and lag in the motor drivers. The mass is very accurate. 


\subsubsection{Cube Mass Properties}

To assess the accuracy of the simulation's mass properties of the cube, the device was released from the upright position both in the simulation and the hardware without an active control logic and the dynamic behaviors were observed. The inertia of the cube was not directly compared because the inertia is different depending on the direction in which the cube falls. Instead, the motions of the cube as calculated by the simulation and as measured by the hardware were compared. The angle $\theta$, which describes the motion of the system is represented in Figure 10.2. The difference in mass can be read from Table 10.2 .

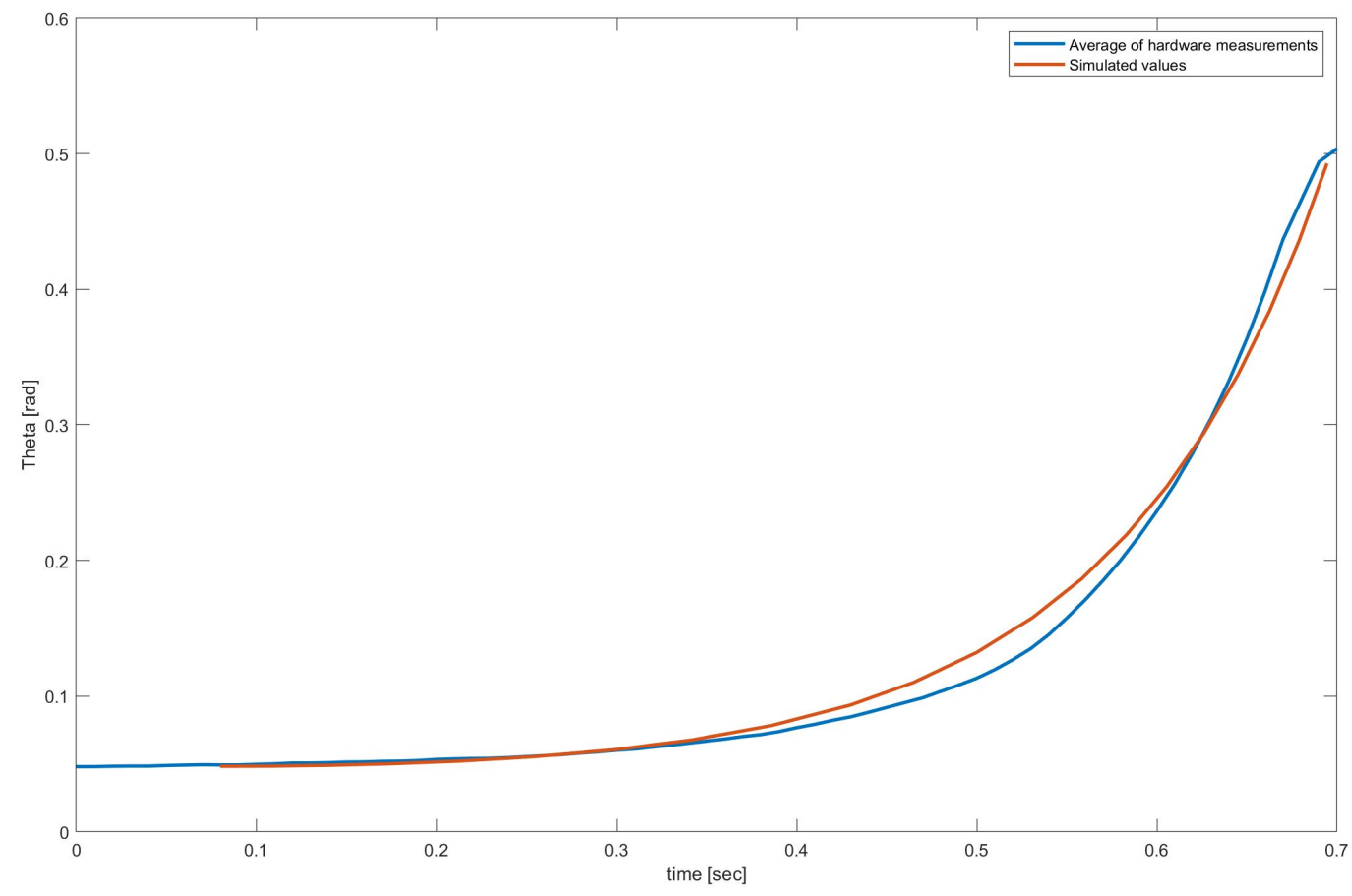

Figure 10.2: The angle $\theta$ of the cube as it falls from its upright position into the same direction as calculated by the simulation and as measured from actual hardware.

\begin{tabular}{c|c|c|c} 
Mass property & Simulation value & Hardware value & Percent difference \\
Mass & $1493 \mathrm{~g}$ & $1512 \mathrm{~g}$ & $1.26 \%$
\end{tabular}

Table 10.2: Mass difference between the simulation and hardware. 


\subsection{Control Algorithm Analysis}

\subsubsection{Functionality of Control Algorithm in Simulation}

The control algorithm developed for the balancing cube functioned very well in the simulation. The results can be seen in Figure 10.3. The control logic successfully balances the cube on a corner when it is released from a few degrees away from this orientation. Additionally, the gains minimize the speeds of the reaction wheels, as shown in Figure 10.4 .

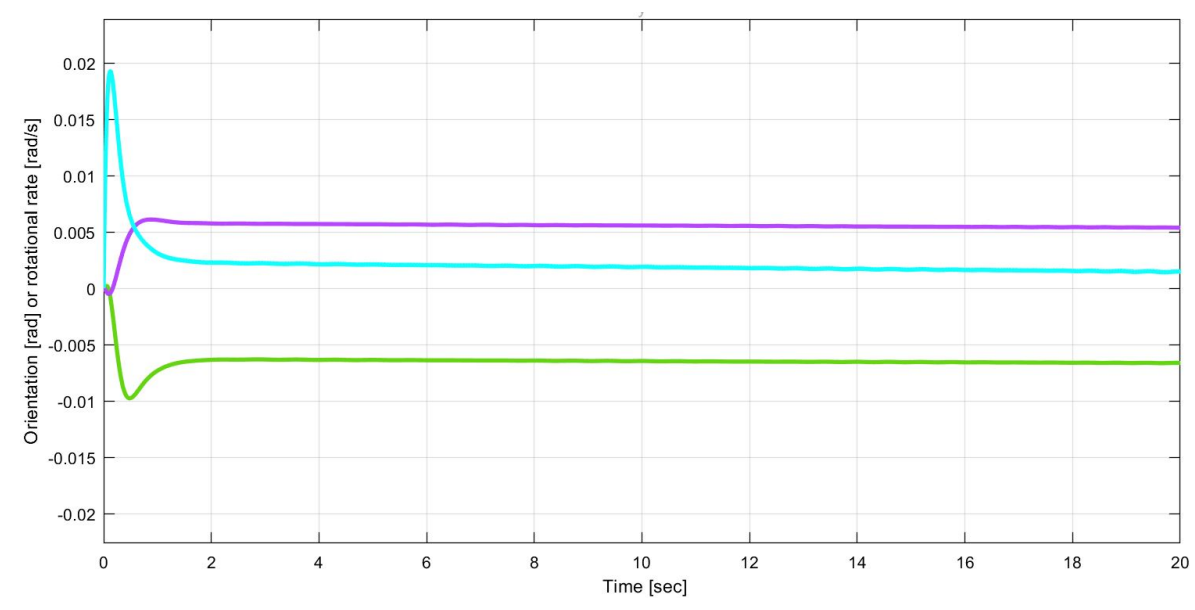

Figure 10.3: Simulated orientation of the cube, given by $\theta$ and $\phi$, and rate of rotation $\dot{\psi}$.

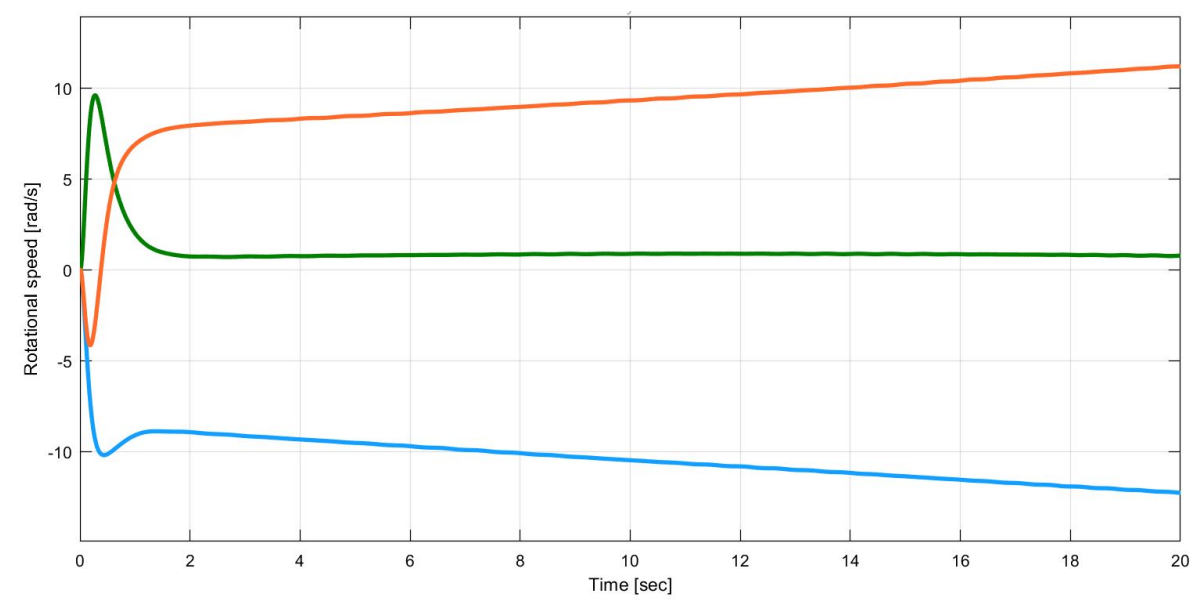

Figure 10.4: Simulated rotational rates of the three reaction wheels. 
The control algorithm demands that the system move towards the upright position while also keeping the wheel speeds low. These goals are not simultaneously achievable since the upright position is inherently unstable and requires a constant torque input. Note that the upright position is the orientation in which the top corner, not the center of gravity, lies directly above the bottom corner. As a result, the algorithm finds a middle ground. The consequence of this is that the orientation angles $\theta$ and $\phi$ converge to non-zero values and the reaction wheel speeds slowly increase, as can be seen in Figures 10.3 and 10.4 respectively. Prioritizing a lower wheel speed results in the cube heading closer to the balancing point while prioritizing the minimization of the cube angles keeps the mechanism closer to the upright position.

\subsubsection{Functionality of Control Algorithm in Hardware}

The implementation of the discrete control logic in actual hardware was not successful. The cube was stood up onto a corner before the control algorithm started running. When it began to operate, the cube did not make the correct effort to balance. Unlike the initial wheel velocity spike seen in the simulation, the reaction wheels barely rotated when the control algorithm was started until they abruptly began to spin to a high rotational velocity.

Figure 10.5 shows the three three states which represent the cube's orientation and rate of rotation. Angles $\theta$ and $\phi$ were relatively close to zero throughout the test because the cube was actively held in the upright position. This was done because the

mechanisms was obviously unstable and would otherwise just have fallen over. Note that $\dot{\psi}$ exceeded the maximum rotational rate of the cube, as described in Chapter 8.3. significantly. 


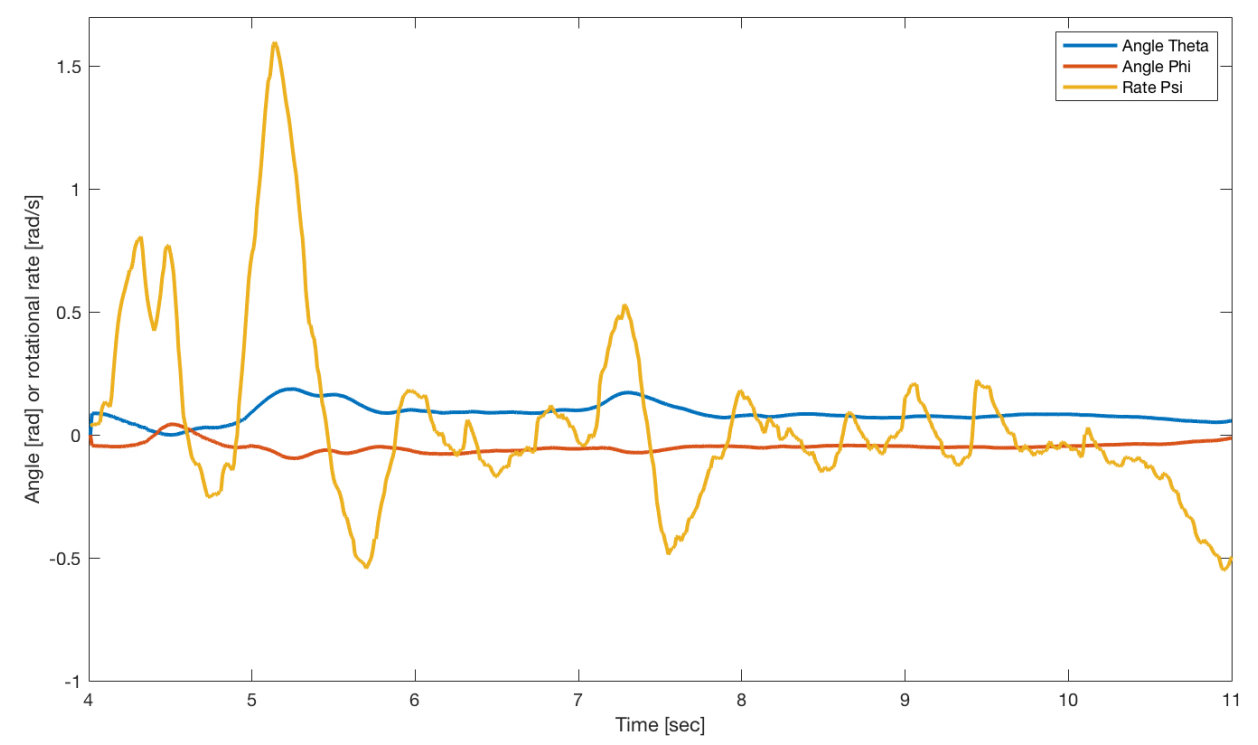

Figure 10.5: Angles $\alpha$ and $\beta$ and rotational rate $\dot{\psi}$ of the cube during active control. The device was held in place to keep it from falling over.

Figure 10.6 shows the rotational rates of the reaction wheels. The wheels do rather little for a fraction of a second before accelerating at the greatest allowable torque until the third reaction wheel spins at 80 radians/second. At this point, the safety stop feature kicks in and the torque input to the wheels is halted. Once the wheel is under the maximum threshold again, the mechanism continues. After this, reaction wheel number one spins up until the software halts the input to the motors. The important thing to note is that the reaction wheel speeds are not driven to zero and therefore the control algorithm does not seem to work properly. 


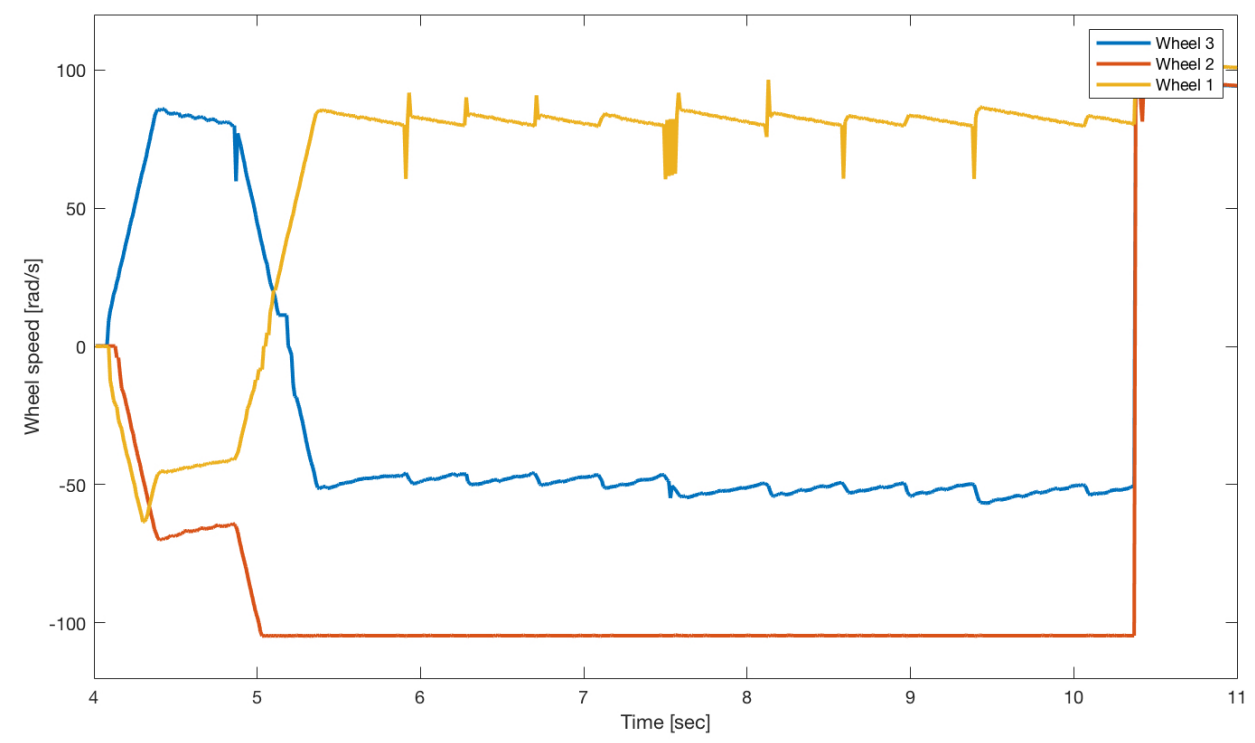

Figure 10.6: Speeds of the three reaction wheels during an active balancing attempt of the cube.

\subsubsection{Analysis of the Discrepancy}

The cause for the discrepancy between the control algorithm running the simulation and the hardware was not fully identified. However, a thorough analysis of the data suggested that the state estimator did not work properly in the hardware. This was discovered by stepping through the data and finding the first unreasonable set of numbers. Starting at the difference between the reference signal $y_{r e f}$ and the output vector $y$, which is shown in Figure 10.7, the signal still looked correct. It is the inverse of the $y$ vector, which makes sense since the error is simply $y$ subtracted from zero. 

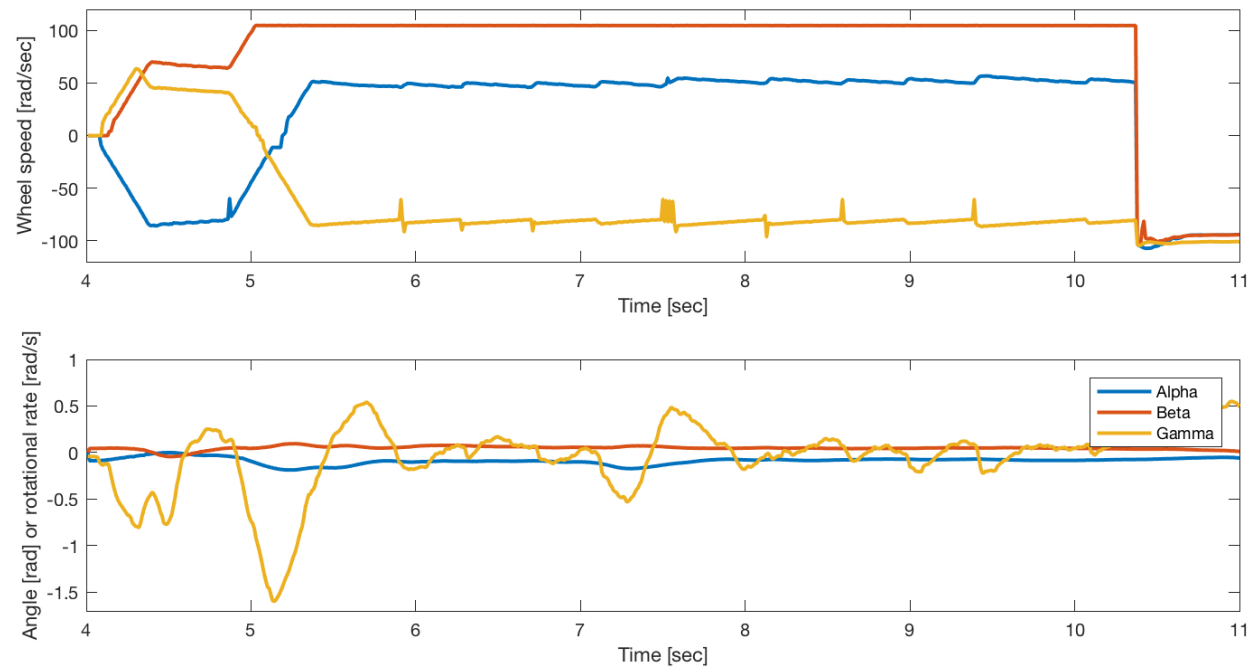

Figure 10.7: The error signal as calculated by the control loop during operation on the hardware.

The result of the state estimation was severely divergent. As can be seen in Figure 10.8, all twelve states of the vector $\hat{x}$ rapidly exceeded their maximum allowable values. Note that the bounds of the angles are significantly larger than the maximum possible angle of \pm 1.57 . This is a clear indication that the state estimator does not work properly.
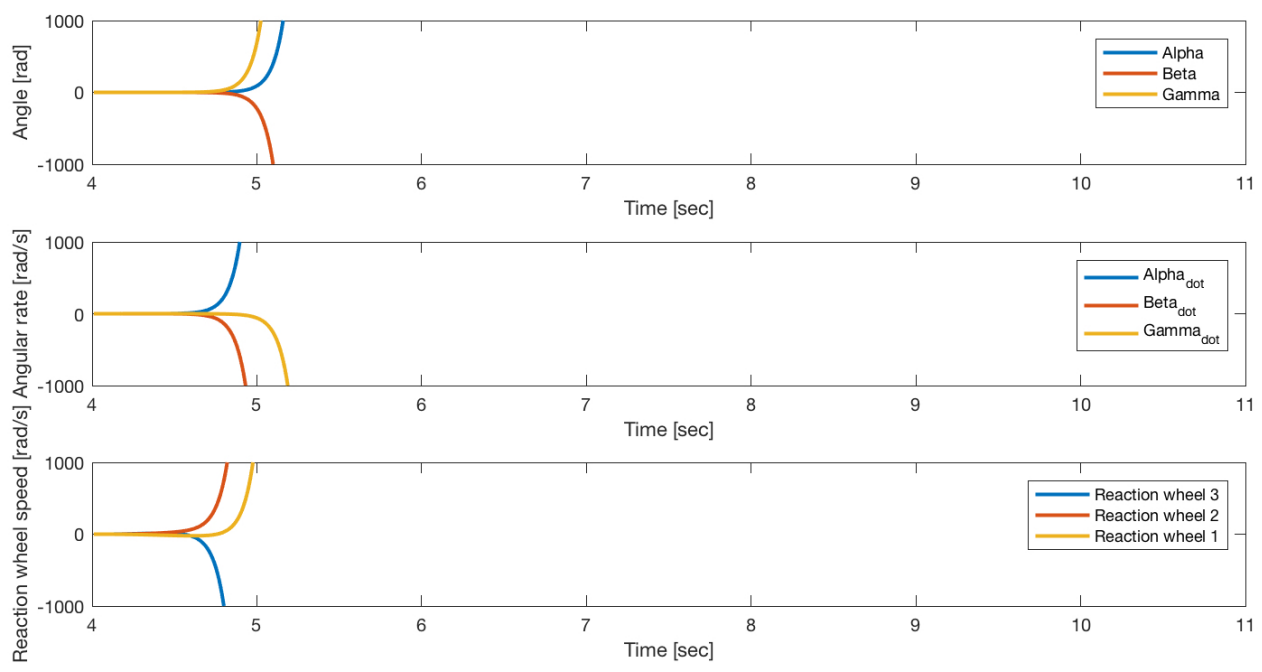

Figure 10.8: The state estimation as calculated by the control loop during operation on the hardware. 
To deep dive further, the results of the multiplication of the L matrix by the error vector between $y_{r e f}$ and $y$ seems to already be different between the simulation and hardware. Figure 10.9 shows these results.
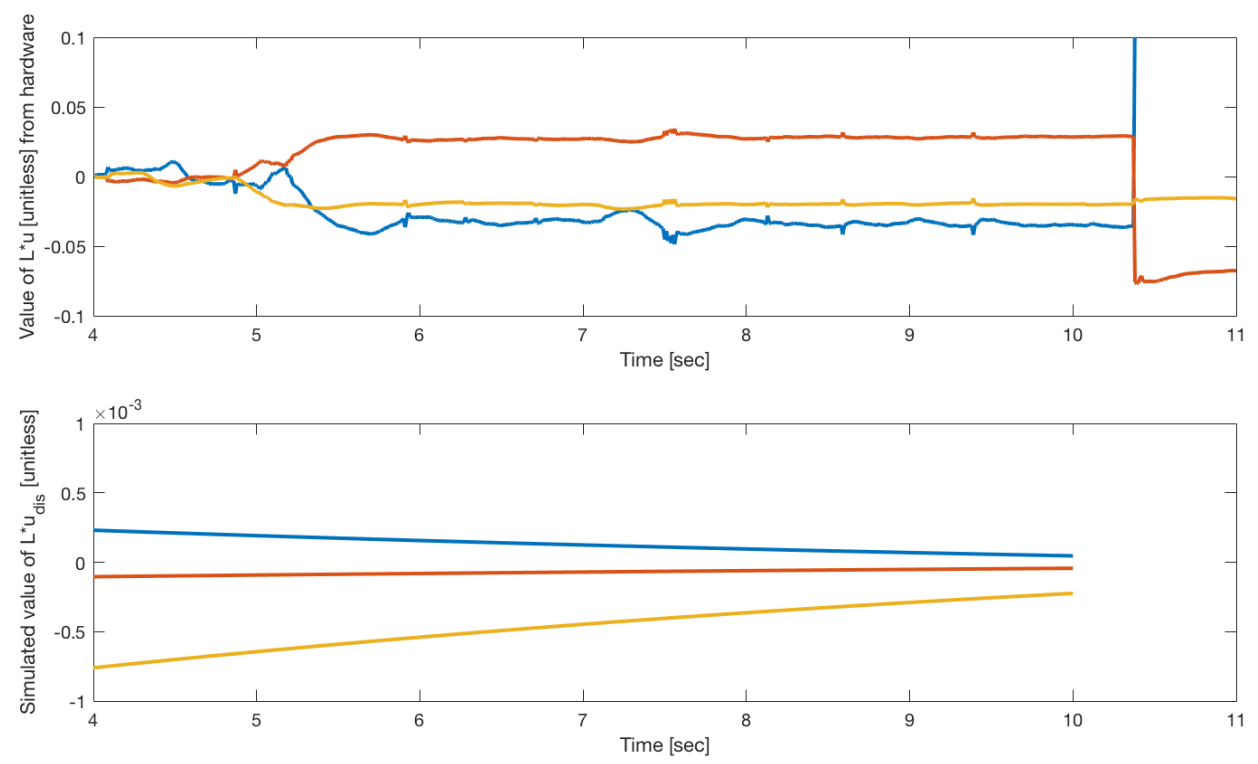

Figure 10.9: Results of the multiplication of the gain $L$ by the error vector. Note that the scales are different.

Since the gain matrix L is the same in the simulation and in the hardware, this discrepancy has to be due to the error vector. Comparing the cube's attitude elements of the error vector, as shown in Figure 10.10, the magnitudes of elements in the simulation are significantly smaller than those in the actual hardware. In other words, the cube starts very close to the desired orientation in the simulations but when the control logic in the hardware begins to operate it registers much larger angles $\theta$ and $\phi$ and rotational rate $\dot{\psi}$ which cause a divergence in the estimation of the state of the machine. 

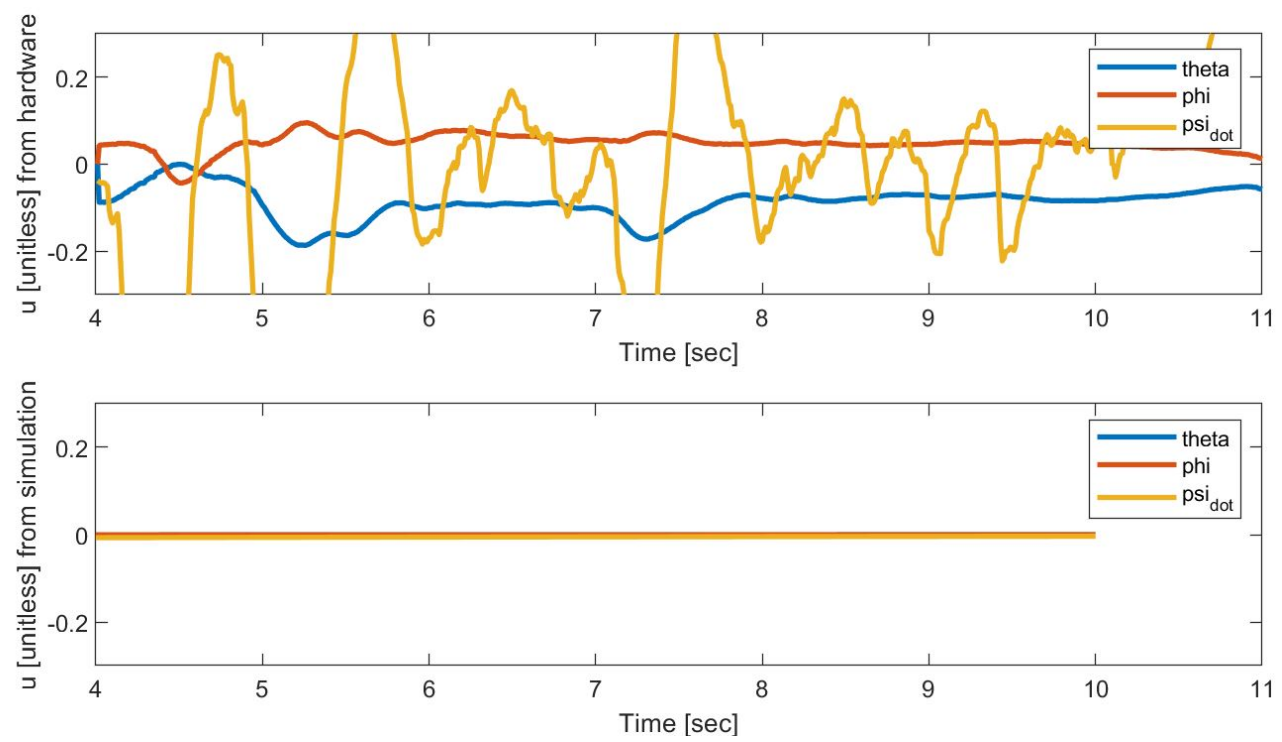

Figure 10.10: Comparison of the error vector as calculated by the simulation and as measured by the hardware. The discrepancy between the two signals is likely the cause for the divergence of the state estimator.

\subsection{Future efforts}

\subsubsection{Adjustment of Control Logic to Balance the Hardware}

Two approaches can be taken to balancing the hardware. The first is a change to the state estimator, the second a modification of the elements in the controlled output vector.

It should be possible to configure the state estimator gains to be able to handle cube attitude signals similar to the ones in Figure 10.10. This likely requires choosing different matrices $Q n, R n$, and possible even $N n$. Another change which may alleviate the issue is the use of the delayed kalman function which, according to MathWorks, is "easier to implement inside control loops" 11.

The other approach is to drive all controllable states of the cube so that the controlled output vector becomes 


$$
y=\left[\begin{array}{c}
\alpha_{\text {cube }} \\
\beta_{\text {cube }} \\
\gamma_{\text {cube }} \\
\dot{\alpha}_{\text {cube }} \\
\dot{\beta}_{\text {cube }} \\
\dot{\gamma}_{\text {cube }} \\
\dot{\theta}_{\text {wheel } 1} \\
\dot{\theta}_{\text {wheel } 2} \\
\dot{\theta}_{\text {wheel } 3}
\end{array}\right]
$$

This change would significantly decrease the reliance on the state estimator, which would only have to estimate the angles of the reaction wheels. These have little to no impact on the actuation of the reaction wheels. However, this change would also mean that the cube would seek to orient itself in a specific way. This in turn could be solved by measuring the orientation of the system before it begins to control its attitude and using these measurements as the reference signal for the control loop. The angles would be driven to nonzero values, requiring a set-point control algorithm.

\subsubsection{Refinement of Simulation}

Some minor adjustments can be made to the simulation to make it more accurate. The model of the bearings could be changed so that the inner races spins with the reaction wheels. The cables could also be modeled in CAD. To take things yet another step further, instead of applying a constant density condition to many of the components, the weight distribution and inertia properties could be more accurately specified. 


\subsubsection{Refinement of Control Algorithm}

To minimize the issue of the control algorithm having two goals which cannot be achieved at the same time, as presented in Section 10.2.1, the plant model could be linearized about the balancing point rather than the upright position. This was not possible in the beginning of the project because the center of gravity of the real hardware was not known. Now that the hardware is complete, this property could be measured and transferred to the simulation.

It may be of importance to inspect the discrepancy between the simulation values

and the hardware measurements which describe the inertia of the reaction wheels. One possible aspects to dive deeper on is the lack of spokes in the animation.

\subsubsection{Refinement of Software}

Some minor refinements could be made to the software as well. The mechanism which stops the controller from spinning the reaction wheels excessively quickly does not work as intended. The software only stops powering the motors when a reaction wheel spins too fast in one of the two directions. It would be advantageous to completely disable the reaction wheels when the threshold speed is reached instead of disabling them while one of the wheels spins too quickly and then enabling them again afterwards.

\subsubsection{Refinement of Hardware}

Improvements to the hardware would make the cube more controllable. The primary suggested modification is to install internal power. Three small batteries should be able to fit into the space between the motors and motor mounts for an optimally low center of gravity and mass symmetry. Another electrical board would be required 
to power the motor drivers and Raspberry Pi at different voltage levels. A major advantage of this modification would be the lack of the tethers which may have a substantial effect on the center of gravity of the system and cause a resistance to changes in the attitude of the cube. Another idea is to add ballast weights to relocate the center of gravity to the geometric centerline of the cube. 
Bibliography

[1] K. Lundberg, "The inverted pendulum system," 1994.

[2] M. Gajamohan, M. Muehlebach, T. Widmer, and R. D'Andrea, "The cubli: A reaction wheel based 3d inverted pendulum," in European Control Conference, 2013.

[3] MathWorks. (). Enable simscape multibody link solidworks plug-in, [Online]. Available: https : / / www . mathworks . com / help / physmod / smlink / ref / linking-and-unlinking-simmechanics-link-software-with-solidworks. html.

[4] —, (). Working with simulink units, [Online]. Available: https : / / www . mathworks . com / help/physmod / simscape /ug / working - with-simulinkunits.html.

[5] J. B. Kuipers, Quaternions and Rotation Sequences: A Primer with Applications to Orbits, Aerospace and Virtual Reality. Aug. 19, 2002.

[6] MathWorks. (). Linearize a plant model for use in feedback control design, MathWorks, [Online]. Available: https://www .mathworks.com/help/physmod/ simscape/ug/linearize-a-plant-model-for-use-in-feedback-controldesign.html.

[7] C.-T. Chen, Linear System Theory and Desgn. 1999.

[8] J. P. Hespanha, Undergraduate lectures notes on lqg/lqr controller design, 2007.

[9] MathWorks. (). Lqr, [Online]. Available: https://www. mathworks.com/help/ control/ref/lqr.html. 
[10] J. P. How, "16.323 principles of optimal control, Massachusetts institute of technology: Mit opencourseware," 2008, [Online]. Available: https : / / ocw . mit.edu.

[11] MathWorks. (). Kalman, [Online]. Available: https ://www . mathworks .com/ help/control/ref/kalman.html.

[12] K. J. Åström, Lecture notes for me155a, 2002.

[13] N. E. Helwig, "Data, covariance, and correlation matrix," Jan. 16, 2017, [Online]. Available: http : //users . stat . umn . edu/ helwig/notes / datamatNotes.pdf.

[14] (). Inverted pendulum: State-space methods to controller design, University of Michigan, [Online]. Available: http://ctms.engin.umich.edu/CTMS/index. php?example=InvertedPendulum\&section=ControlStateSpace\#6.

[15] MathWorks. (). Linearize a plant model for use in feedback control design, MathWorks, [Online]. Available:https://www.mathworks.com/help/physmod/ simscape/ug/linearize-a-plant-model-for-use-in-feedback-controldesign.html.

[16] R. M. Murray, "Lecture 2 - lqr control," Jan. 11, 2006, [Online]. Available: https://www.cds.caltech.edu/ murray/courses/cds110/wi06/lqr.pdf.

[17] 1. a. Adafruit. (). Adafruit 16-channel pwm/servo hat \& bonnet for raspberry pi, [Online]. Available: https ://learn . adafruit . com/adafruit-16-channelpwm-servo-hat-for-raspberry-pi?view=all.

[18] N. Semiconductors, Pca9685, version 4, NXP Semiconductors, Apr. 2015.

[19] J.-C. Wippler. (). I2c on the raspberry pi, JEELABS, [Online]. Available: https: //jeelabs.org/book/1452c/. 
[20] D. Z. Maze. (). I2cdump - linux man page. M. D. S. Frodo Looijaard and J. Delvare, Eds., die.net, [Online]. Available: https ://linux.die.net/man/8/ i2cdump.

[21] MathWorks. (2016). Control a raspberry pi powered robot with matlab and simulink, [Online]. Available: https ://www . mathworks . com/matlabcentral/ fileexchange / 47376-control-a-raspberry-pi-powered-robot-withmatlab-and-simulink.

[22] B. Jones. (2015). Change i2c speed with raspberry pi, mindsensors.com, [Online]. Available: http://www . mindsensors . com/blog/how-to/change-i2cspeed-with-raspberry-pi.

[23] MathWorks. (). Control signal data types, [Online]. Available: https://www . mathworks.com/help/simulink/ug/control-signal-data-types.html.

[24] T. Instruments, Ads101x ultra-small, low-power, i2c-compatible, 3.3-ksps, 12-bit adcs withinternal reference, oscillator, and programmablecomparator, 2009.

[25] Bosch, Bno055 intelligent 9-axis absolute orientation sensor, version 1.4, 2016.

[26] M. Motor, Escon 36/3 ec hardware reference, 2015.

[27] SDSU-EDORAS. (Jun. 2002). How simulink works, [Online]. Available: https: //edoras .sdsu . edu/doc/matlab/toolbox/simulink/ug/how_simulink_ works6.html.

[28] D. Rowell. (). Discrete time observers and lqg control, [Online]. Available: http: //web.mit.edu/2.151/www/Handouts/Kalman.pdf.

[29] MathWorks. (). Control and display the sorted order, [Online]. Available: https: //www . mathworks. com/help/simulink/ug/controlling-and-displayingthe-sorted-order.html. 
[30] missile. (Jan. 26, 2004), [Online]. Available: https : //www . opengl . org / discussion_boards/archive/index.php/t-159866.html. 


\title{
APPENDICES
}

\author{
Appendix A
}

MATLAB CODE

\section{Felix Haimerl}

\section{$03 / 01 / 18$}

clear all; clc

\section{Model Parameters}

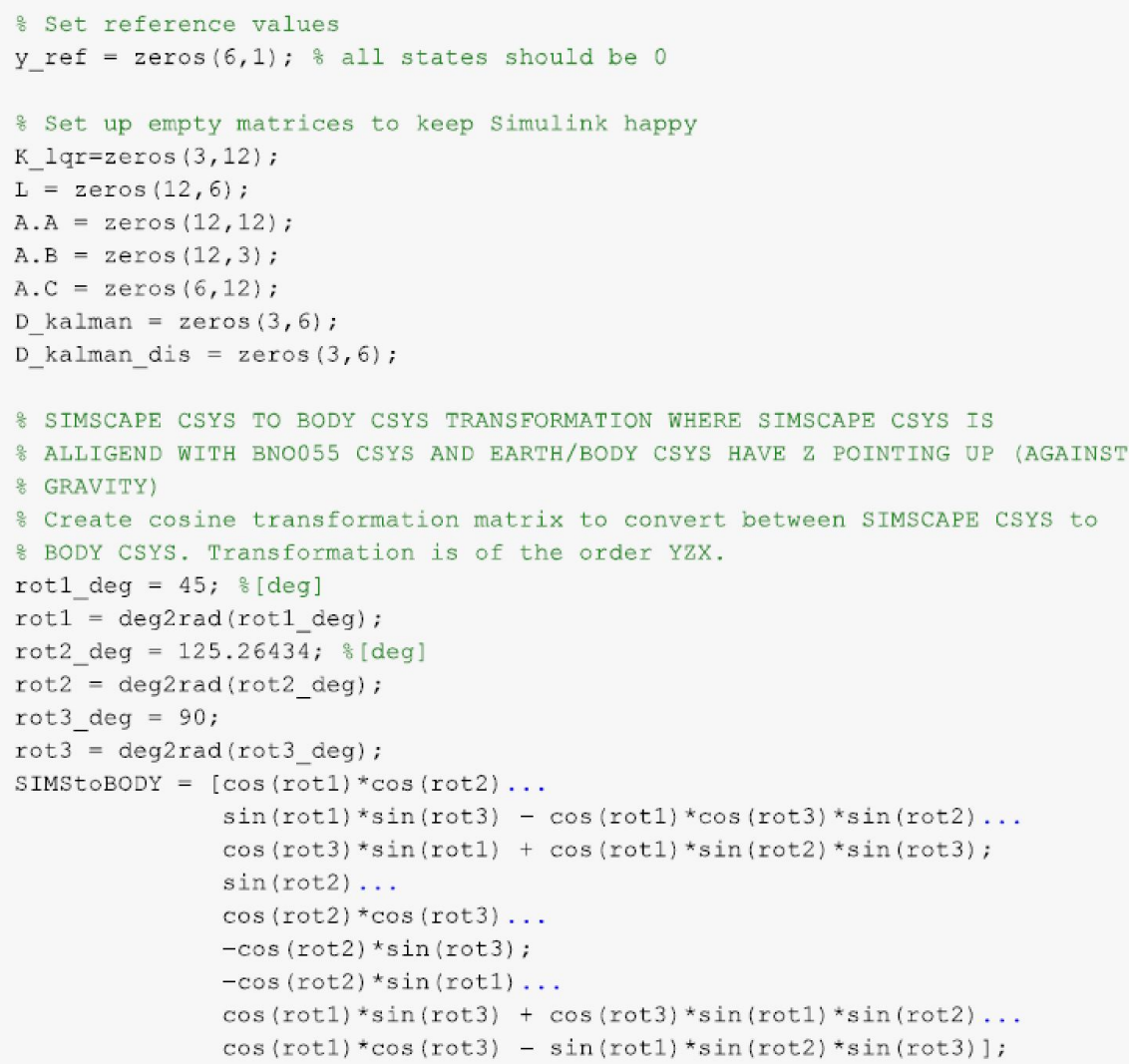




\section{Linearizing system}

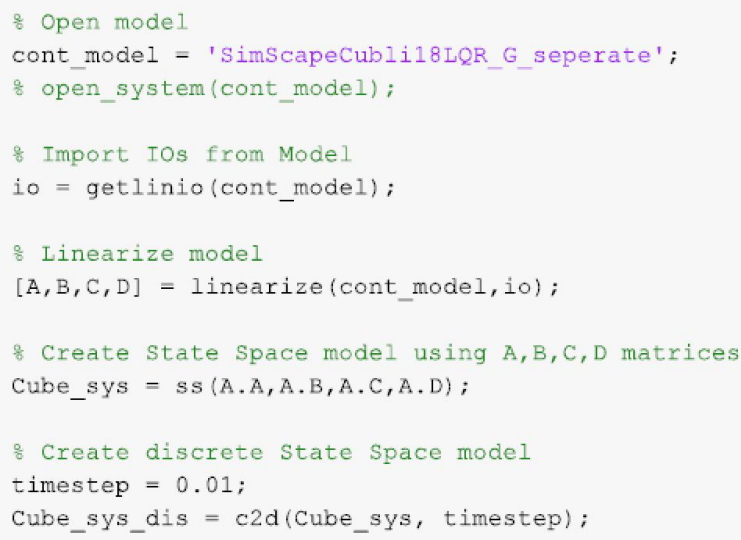

\section{LQR gain for discrete system}

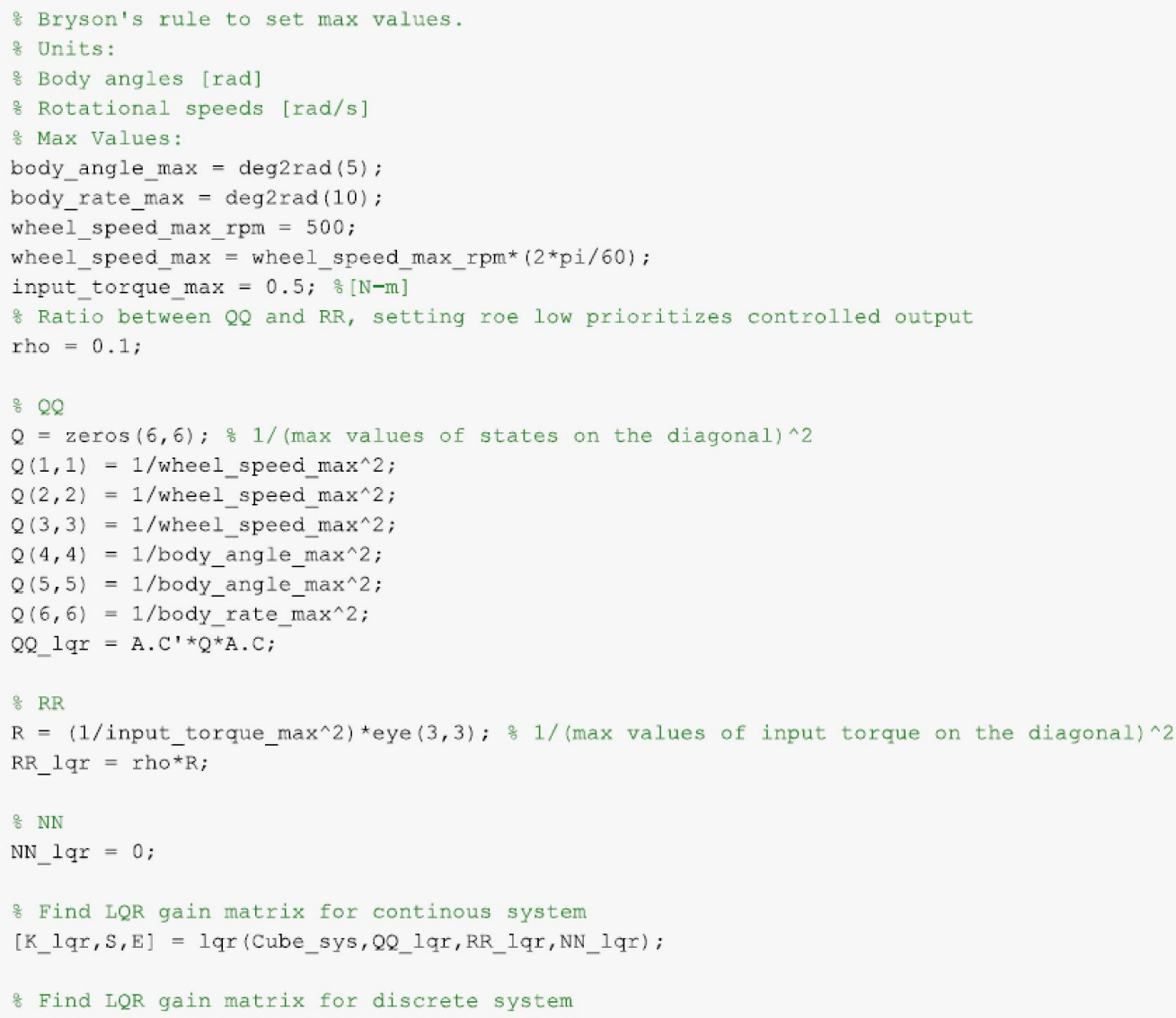


[K_lqr_dis,s_dis,E_dis] = lqr (Cube_sys_dis, QQ_lqr,RR_lqr, NN_lqr);

\section{State estimation and kalman filtering for discrete system}

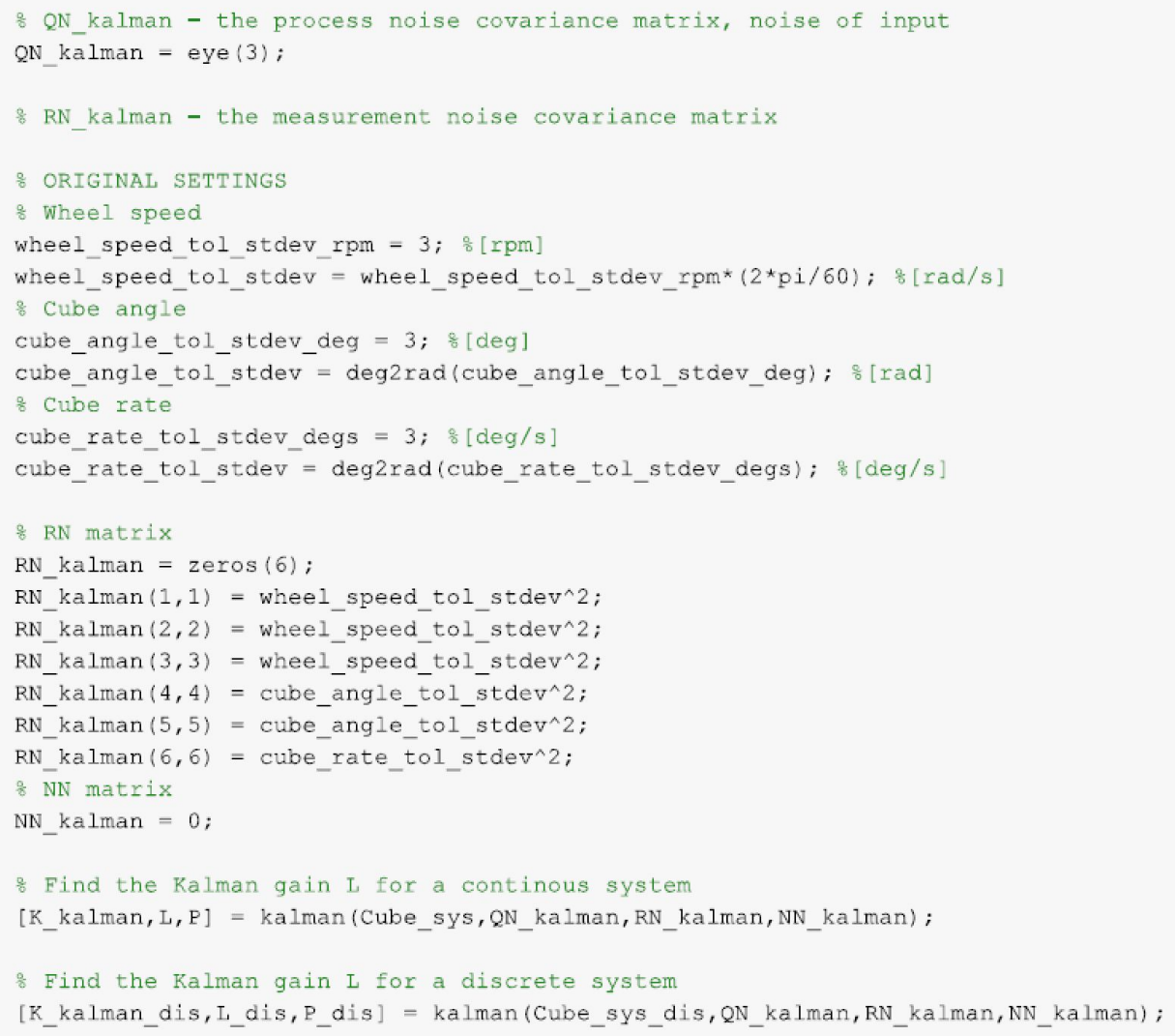

\section{Simulate model}

s simulate the continuous system with the given parameters sim(cont_model)

\& simulate the discrete system with the given parameters dismodel = 'DiscreteSimulation'; $\operatorname{sim}$ (dismodel)

Published with MATLAB@ R2018a 